\title{
Unconventional Superconductivity in Heavy-Fermion Compounds
}

\author{
B. D. White ${ }^{\mathrm{a}, \mathrm{b}}$, J. D. Thompson ${ }^{\mathrm{c}}$, M. B. Maple $\mathrm{T}^{\mathrm{a}, \mathrm{b}, *}$ \\ ${ }^{a}$ Department of Physics, University of California, San Diego, La Jolla, CA 92093, USA \\ ${ }^{b}$ Center for Advanced Nanoscience, University of California, San Diego, La Jolla, CA 92093, USA \\ ${ }^{c}$ Los Alamos National Laboratory, Los Alamos, New Mexico 87545, USA
}

\begin{abstract}
Over the past 35 years, research on unconventional superconductivity in heavy-fermion systems has evolved from the surprising observations of unprecedented superconducting properties in compounds that convention dictated should not superconduct at all to performing explorations of rich phase spaces in which the delicate interplay between competing ground states appears to support emergent superconducting states. In this article, we review the current understanding of superconductivity in heavy-fermion compounds and identify a set of characteristics that is common to their unconventional superconducting states. These core properties are compared with those of other classes of unconventional superconductors such as the cuprates and iron-based superconductors. We conclude by speculating on the prospects for future research in this field and how new advances might contribute towards resolving the long-standing mystery of how unconventional superconductivity works.
\end{abstract}

Keywords:

unconventional superconductivity, heavy-fermion behavior, quantum critical point, non-Fermi liquid behavior, magnetic fluctuations, Kondo lattice

\section{Introduction}

In the two decades prior to the 1979 discovery of unconventional superconductivity in $\mathrm{CeCu}_{2} \mathrm{Si}_{2}[1]$, the properties of superconductors were typically studied within the context of the Bardeen-Cooper-Schrieffer (BCS) theory of superconductivity $[2,3,4]$. The BCS theory describes pairing between conduction electrons as being mediated by phonons so that the characteristic Debye temperature, $\Theta_{D}$, plays an important role as an energy scale. However, the unexpected discovery of superconductivity in $\mathrm{CeCu}_{2} \mathrm{Si}_{2}$ turned this paradigm on its head. The superconducting Cooper pairs in $\mathrm{CeCu}_{2} \mathrm{Si}_{2}$ are not formed from ordinary conduction electrons, but are rather composed of quasiparticles with enhanced effective masses [1]. Furthermore, the hierarchy of energy scales departs significantly from what is assumed in the BCS theory, indicating that pairing of the quasiparticles cannot be phonon-mediated. These observations made it clear that $\mathrm{CeCu}_{2} \mathrm{Si}_{2}$ was no ordinary superconductor, and heralded a new era of research on unconventional superconductivity. The course of this research has evolved into current explorations of rich phase spaces wherein new and exciting physics has emerged from tuning the delicate interplay between competing ground states. Before discussing the unconventional superconducting states in these fascinating materials, which is the subject of this review, we first briefly introduce the characteristics and origin of heavy-fermion compounds.

*Corresponding author: mbmaple@ucsd.edu

\subsection{Heavy-Fermion Compounds}

The physical properties of simple metals such as the elements sodium or copper can be understood well by models that neglect the many-body interactions that occur between electrons. In even slightly more complex materials, this unrealistic assumption fails dramatically, but the itinerant electron states and their interactions with other electrons can be transformed into non-interacting quasiparticles with enhanced effective masses by invoking the Fermi liquid theory [5, 6, 7]. A Fermi liquid state, which is populated by these independent quasiparticles, is characterized by properties that include a quadratic temperature dependence of the electrical resistivity, $\rho(T) \sim\left(T / T_{F}\right)^{2}$ for $T \ll T_{F}$, where $T_{F}=\epsilon_{F} / k_{B}$ is the Fermi temperature and $\epsilon_{F}$ is the Fermi energy [8]. Such a quadratic temperature dependence was first experimentally resolved in 1975 by Andres et al. in measurements on the compound $\mathrm{CeAl}_{3}$ [9]. While $T_{F}$ values for simple metals such as copper are of order $10^{4} \mathrm{~K}[10]$ and a $\left(T / T_{F}\right)^{2}$ temperature dependence of $\rho$ is very difficult to resolve experimentally, analysis of $\rho(T)$ and other physical properties indicated that $T_{F}$ was three orders of magnitude lower (i.e., 15$25 \mathrm{~K}$ ) in $\mathrm{CeAl}_{3}$ [9]! Furthermore, $T_{F}$ is inversely proportional to the coefficient of the electronic contribution to specific heat, $\gamma$, which is proportional to the electronic density of states at $\epsilon_{F}, \mathcal{N}\left(\epsilon_{F}\right)$, and is roughly $0.7 \mathrm{~mJ} \mathrm{~mol}^{-1} \mathrm{~K}^{-2}$ for copper [10] compared to $\gamma \simeq 1600 \mathrm{~mJ} \mathrm{~mol}^{-1} \mathrm{~K}^{-2}$ for $\mathrm{CeAl}_{3}$ [9]. Instead of observing magnetic order within the lattice of localized magnetic moments associated with trivalent Ce ions separated by $\sim 4 \AA$, a nonmagnetic Fermi-liquid ground state, populated by quasiparticles of order 1000 times heavier than free electrons, was observed in $\mathrm{CeAl}_{3}$. These astounding results hinted at an 
enormous increase in $\mathcal{N}\left(\epsilon_{F}\right)$ as well as the emergence of a new characteristic energy scale in $\mathrm{CeAl}_{3}$ that acts as an effective $T_{F}$.

To understand how these remarkable properties are possible, it is instructive to first consider what happens when a single impurity bearing a localized magnetic moment is dissolved into a non-magnetic metallic host. The Friedel-Anderson model was developed principally to explain magnetic moment formation in metals, but it also describes such a single-ion impurity scenario [11]. In this model, quantum mechanical admixing or hybridization between the localized electron states of the impurity ion and the itinerant electron states from the host, characterized by hybridization matrix element $\mathcal{V}$, produces a virtual bound state or resonance in the density of electronic states with width $\Gamma \propto \mathcal{V}^{2}$ [11]. When the energy associated with this resonance (the maximum) is within $\Gamma$ of $\epsilon_{F}, \mathcal{N}\left(\epsilon_{F}\right)$ is enhanced.

Addressing a different problem (at least superficially), Jun Kondo proposed a simple Hamiltonian to explain the logarithmic divergence with decreasing temperature observed since the 1930's [12] in measurements of $\rho(T)$ on systems containing dilute magnetic impurities. The essential physics of the Kondo Hamiltonian is embodied in a magnetic exchange coupling $\mathcal{J}$ between the spin $\vec{S}$ of a magnetic impurity with the local spin density of the surrounding conduction electrons $\overrightarrow{s_{0}}$. Using this model and the second Born approximation [13], Kondo was able to derive the long sought after $\rho(T) \sim-\log (T)$ behavior. More importantly for our discussion, a new energy scale, designated the Kondo temperature $T_{K}$, also emerged from these calculations [13], which separates the temperature region over which the calculations are valid $\left(T>T_{K}\right)$ from $T<T_{K}$ where perturbation theory breaks down. The Friedel-Anderson and Kondo models, which contribute an enhancement of $\mathcal{N}\left(\epsilon_{F}\right)$ and a new energy scale $T_{K}$, respectively, are equivalent descriptions of a magnetic impurity in a metallic host as shown by Schrieffer and Wolff [14]. The antiferromagnetic exchange interaction of the Kondo model $\mathcal{J}$ is calculable in terms of $\mathcal{V}$ from the Friedel-Anderson Hamiltonian,

$$
\mathcal{J}=2 \mathcal{V}^{2}\left(1 /\left|\epsilon_{f}\right|+1 /\left(\epsilon_{f}+U\right)\right)
$$

where $\epsilon_{f}=\epsilon_{i}-\epsilon_{F}$ and $\epsilon_{i}$ is the energy of the localized electrons in the unfilled shell of the magnetic impurity ion [14].

A physical picture of how a magnetic impurity interacts with its surroundings emerges from these theoretical developments. At temperatures comparable to but greater than $T_{K} \propto$ $\exp \left[-1 /\left(\mathcal{N}\left(\epsilon_{F}\right) \mathcal{J}\right)\right][15]$, the antiferromagnetic exchange interaction, characterized by $\mathcal{J}$, between $\vec{S}$ and $\overrightarrow{s_{0}}$ promotes polarizing $\overrightarrow{s_{0}}$ antiparallel to the direction of $\vec{S}$. This tendency causes the localized magnetic moment of the impurity ion to become dynamically screened or compensated, and the exchange interaction leads to strong local spin-flip or incoherent Kondo scattering of charge carriers (i.e., a $\rho(T) \propto-\log (T)$ term is obtained in this temperature regime). At temperatures less than $T_{K}$, the screening of the impurity ion's magnetic moment becomes complete as the surrounding conduction electrons form a bound state with the impurity, producing a single non-magnetic quasiparticle with an enhanced effective mass. This many-body singlet state behaves like a local Fermi liquid [16], emphasizing that $T_{K}$ is a suitable effective $T_{F}$.
The physics of a single magnetic impurity embedded in a metallic host, which is treated in great detail elsewhere $[17,18,19,15]$, provides a mechanism for an enhanced $\mathcal{N}\left(\epsilon_{F}\right)$ via the emergence of a quasiparticle resonance and leads to a new energy scale $T_{K}$, below which, a local Fermi-liquid state exists. However, heavy-fermion materials like $\mathrm{CeAl}_{3}$ do not contain dilute concentrations of magnetic impurities, but are rather composed of a lattice of localized magnetic moments coupled to the Fermi sea [19]. These compounds share other common traits including a crossover from Curie-Weiss behavior at high temperature to enhanced Pauli magnetic susceptibility at low temperature and a broad peak-like feature in the electrical resistivity. These and other characteristics of heavy-fermion compounds can be understood within the context of a generalization of the Friedel-Anderson model called the periodic Anderson model. In an overly simplistic picture, the periodic Anderson model assumes that the Kondo effect occurs at every site bearing a localized magnetic moment. In principle, this leads to the formation, below an energy scale $T^{*}$, of $10^{23}$ quasiparticles per mole localized magnetic moments. Characteristic Kadowaki-Woods [20, 21] and Wilson-Sommerfeld [17] ratio values, calculated using quantities obtained from experimental data below $T^{*}$, are used to confirm Fermi-liquid ground states. $T^{*}$, sometimes referred to as the Kondo lattice coherence temperature, is typically identified with the temperature of the maximum of the peak-like feature in $\rho(T)$ and is distinct from $T_{K}$. The crossover from localized to delocalized magnetic-moment behavior in the magnetic susceptibility also occurs near $T^{*}$. In many cases, $T^{*}<T_{K}$ such that screening of the localized magnetic moments begins near $T_{K}$, but is incomplete until $T^{*}$ when the Kondo lattice becomes coherent. However, under certain filling conditions [22] or when mutual screening of local magnetic moments enhances $T^{*}$ relative to $T_{K}$ [23, 24, 25], cases where $T^{*}>T_{K}$ have been reported and discussed.

The periodic Anderson model is particularly well suited to describing the properties of metallic compounds containing sublattices of $\mathrm{Ce}, \mathrm{Sm}, \mathrm{Yb}, \mathrm{U}$, and $\mathrm{Pu}$ ions. This is largely a consequence of these ion's tendency towards valence instabilities in metals [26] that allows for a finite $T_{K}$ and $T^{*}$ (i.e., $\epsilon_{f}$ is small enough so that $\mathcal{J} \propto \mathcal{V}^{2} /\left|\epsilon_{f}\right|$ is finite for reasonable values of $\mathcal{V}$ ). It is natural then that the majority of known heavyfermion compounds are composed of one of these lanthanide or actinide elements, with Ce- and U-based compounds being particularly well represented. There are also a more limited number of Pr-based heavy-fermion compounds that are known, and these sometimes involve the quadrupolar Kondo effect that was originally proposed for compounds containing $\mathrm{U}^{4+}$ ions with cubic point group symmetry [27, 28, 29]. The electric quadrupole moment, associated with a non-magnetic $\Gamma_{3}$ doublet ground state of $\mathrm{Pr}^{3+}$, can be screened just like a magnetic moment in the conventional Kondo effect, leading to a heavyfermion state [30, 31]; however, we note that the heavy-fermion superconductor $\mathrm{PrOs}_{4} \mathrm{Sb}_{12}$ serves as a counter-example with its nonmagnetic singlet $\Gamma_{1}$ ground state $[32,33]$.

With the exception of $\mathrm{Sm}$, the total angular momentum $J$ ground states for these lanthanide and actinide ions are generally not significantly admixed with excited states [34], but 
the $2 J+1$-fold degeneracy associated with $J$ is usually at least partially lifted by the crystalline electric field (CEF). Since the unfilled $4 f$ or $5 f$ electron shells of these ions are so well screened by the surrounding filled electron shells, the energy splitting $\delta$ between the ground and first excited states of the multiplet can be comparable to energy scales such as $T_{K}, T^{*}$, or the superconducting critical temperature $T_{c}$. Furthermore, the hybridization between itinerant and localized electron states, characterized by $\mathcal{J}$, also facilitates the indirect RudermanKittel-Kasuya-Yosida (RKKY) magnetic exchange interaction $[35,36,37]$ that couples the localized magnetic moments [15]. In the context of the simple and intuitive Doniach phase diagram [38], $\mathcal{J}$ is the parameter that determines whether the heavy Fermi liquid or the magnetically-ordered (usually antiferromagnetic) ground state overcomes the other. Therefore, the physical properties of these materials are governed by the delicate interplay of comparable energy scales and competition, or sometimes coexistence, between distinct ground states. A rich phase space emerges from this environment, containing numerous ground states often with quantum critical points residing at their zero-temperature boundaries and with nearby quantum criticality at finite temperatures [38, 8, 39]. Looking beyond $f$-electron systems, we note that there are reports of at least two $d$-electron transition-metal oxides, $\mathrm{LiV}_{2} \mathrm{O}_{4}$ [40] and $\mathrm{CaCu}_{3} \mathrm{Ru}_{4} \mathrm{O}_{12}$ [41], that exhibit characteristics consistent with a heavy-fermion state. Neither of these compounds is superconducting, but transition-metal oxide-based heavy-fermion compounds may present an unexplored frontier of heavy-fermion research.

Though we have only skimmed the surface of this fascinating subject, it is beyond our scope to provide further depth. We refer the interested reader to numerous review articles dedicated to covering heavy-fermion physics $[42,43,44,45,46,47,19$, $48,49]$. Having briefly introduced the fundamentals of heavyfermion physics, we are prepared to consider the basic characteristics of superconductivity in these systems.

\subsection{Superconductivity in Heavy-Fermion Compounds}

Chronologically speaking, the first observation of superconductivity in a heavy-fermion compound occurred in measurements by Bucher et al. on $\mathrm{UBe}_{13}$ [50]; however, the ground state of $\mathrm{UBe}_{13}$ was not considered to be a heavy Fermi liquid at the time and the observed superconductivity, after extensive experiments exercising due diligence, was dismissed by the authors as extrinsic [50]. The observed superconducting transition temperature of $T_{c}=0.97 \mathrm{~K}$ was only reduced by about $0.3 \mathrm{~K}$ in a $6 \mathrm{~T}$ magnetic field [50] (meaning that the initial slope of the upper critical field, $\left(d H_{c 2} / d T\right)_{T_{c}} \simeq-20 \mathrm{~T} / \mathrm{K}$, was improbably large). Even though a diamagnetic signal was obtained from a powdered sample of $\mathrm{UBe}_{13}$, the authors interpreted their results as indicating that superconductivity must arise from precipitated filaments of $U$ [50].

Four years later, Steglich et al. reported superconductivity in $\mathrm{CeCu}_{2} \mathrm{Si}_{2}$ at $T_{c} \simeq 0.51(4) \mathrm{K}$ [1]. It was recognized at that time that $\mathrm{CeCu}_{2} \mathrm{Si}_{2}$ was a heavy-fermion compound, and significant effort was invested on trying to convince the scientific community that the observed superconductivity in $\mathrm{CeCu}_{2} \mathrm{Si}_{2}$ was intrinsic [1]. Early on, two important observations were made that became hallmark characteristics of heavy-fermion superconductors. First, the size of the jump at $T_{c}$ in specific heat data, $\Delta C$, is comparable to $\gamma T_{c}$, indicating that the Cooper pairs are formed from heavy quasiparticles rather than bare conduction electrons. Second, as was the case with $\mathrm{CeAl}_{3}$, the effective $T_{F}$ energy scale is anomalously low; for $\mathrm{CeCu}_{2} \mathrm{Si}_{2}$, the spin-fluctuation temperature of order $10 \mathrm{~K}$ serves as $T_{F}$. The resulting hierarchy of energy scales $T_{c}<T_{F}<\Theta_{D}$ is drastically different from that assumed in the BCS theory of superconductivity $[2,3,4]$ wherein characteristic frequencies obey $k_{B} \Theta_{D} / h \ll k_{B} T_{F} / h$. This observation indicates that superconductivity in $\mathrm{CeCu}_{2} \mathrm{Si}_{2}$ cannot be conventional (i.e. similar to the superfluid state of ${ }^{3} \mathrm{He}$ [51], this was a second example of a system in which pairing cannot be phonon-mediated).

The results for $\mathrm{CeCu}_{2} \mathrm{Si}_{2}$ were initially controversial, and as in most burgeoning subjects in materials physics, it took time to sort out questions of sample quality and other details [42]. However, the results stood the test of time and heavy-fermion superconductors such as $\mathrm{UBe}_{13}$ (the previously-observed superconductivity was demonstrated to be intrinsic) [52], $\mathrm{UPt}_{3}$ [53], and $\mathrm{URu}_{2} \mathrm{Si}_{2}[54,55,56]$ were subsequently discovered and studied. Many of the early discoveries involved a superconducting state that emerged from a non-magnetic heavy Fermi liquid. However, a new era of research was heralded by the discovery of superconductivity in $\mathrm{CePd}_{2} \mathrm{Si}_{2}$ and $\mathrm{CeIn}_{3}$ that appeared only after an antiferromagnetic phase was suppressed by applied pressure $[57,58]$. It is widely believed that fluctuations associated with a magnetic quantum critical point play a role in facilitating the observed non-Fermi liquid behavior in the normal states of these compounds and also serve as the pairing mechanism supporting superconductivity [58]. This picture is expected to apply more broadly to all heavy-fermion superconductors; even the compounds for which superconductivity is observed at ambient pressure generally reside in close proximity to a nearby magnetic or quadrupolar ordered phase that nature has already suppressed.

The superconducting states of heavy-fermion compounds are generally characterized by: (1) $T_{c}$ values usually of order $1 \mathrm{~K}$ or lower, (2) Cooper pairs formed from heavy quasiparticles as inferred from large jumps of order $\gamma T_{c}$ in specific heat at $T_{c} ;$ (3) Large orbital upper critical fields at $0 \mathrm{~K}, H_{c 2}^{*}(0)$, that can be calculated using Werthamer-Helfand-Hohenberg (WHH) theory [59] from the very large initial slopes, $\left(d H_{c 2} / d T\right)_{T_{c}}$, near $T_{c}$; (4) Short superconducting coherence lengths $\xi$ relative to the London magnetic penetration depth $\lambda$ such that the GinzburgLandau parameter $\lambda / \xi>>1 / \sqrt{2}$ (i.e., these are extreme type-II superconductors); (5) Line or point nodes in the superconducting energy gap $\Delta(\vec{k})$; (6) Nearby ordered state in phase space such as magnetic or quadrupolar order. In addition to these ubiquitous characteristics, some of the heavy-fermion superconductors such as $\mathrm{UPt}_{3}$ and $\mathrm{PrOs}_{4} \mathrm{Sb}_{12}$ exhibit evidence for multiple superconducting energy gaps [49].

There are many review articles that cover superconductivity in heavy-fermion compounds [42, 43, 60, 44, 45, 61, 46, $47,19,48,49]$. This review is organized by lanthanide or ac- 
tinide element. Each section focuses primarily on the seminal experimental discoveries, but our narrative is "dressed" by theoretical developments and concepts when appropriate. Additional emphasis is placed on more recently discovered heavyfermion superconductors that have not been covered in earlier review articles including in new Pr- [31, 62] and Yb-based [63] compounds. The family of " 115 " heavy-fermion superconductors is also discussed with extra emphasis due to the many ground-breaking discoveries made in the course of studies on these and related materials over the last roughly 13 years. A core set of empirically-observed characteristics associated with heavy-fermion superconductors are then identified and compared with properties of related "cousins" including unconventional superconductivity in the cuprates and the iron pnictdides/calchogenides. We conclude with a few thoughts concerning the current state of the field of heavy-fermion superconductivity and provide an outlook for future research and developments.

\section{Ce-based heavy-fermion superconductors}

\section{1. $\mathrm{CeM}_{2} \mathrm{X}_{2}(M=\mathrm{Ni}, \mathrm{Cu}, \mathrm{Rh}, \mathrm{Pd} ; \mathrm{X}=\mathrm{Si}, \mathrm{Ge})$}

There is a series of isostructural heavy-fermion superconductors that form with the $\mathrm{ThCr}_{2} \mathrm{Si}_{2}$-type crystal structure with space group $14 / \mathrm{mmm}$. Among this series, superconductivity was discovered in the heavy-fermion compound $\mathrm{CeCu}_{2} \mathrm{Si}_{2}$ at ambient pressure [1]; in contrast, superconductivity emerges only after antiferromagnetic order is suppressed by applied pressure in the compounds $\mathrm{CeCu}_{2} \mathrm{Ge}_{2}$ [64], $\mathrm{CePd}_{2} \mathrm{Si}_{2}$ [57], and $\mathrm{CeRh}_{2} \mathrm{Si}_{2}$ [65]. Interestingly, an antiferromagnetic spin-density wave (SDW) order can be stabilized in slightly $\mathrm{Cu}$-deficient samples of $\mathrm{CeCu}_{2} \mathrm{Si}_{2}$ [66]; this observation indicates that stoichiometric $\mathrm{CeCu}_{2} \mathrm{Si}_{2}$ resides on the border of antiferromagnetic order and suggests that the chemical pressure produced by filling $\mathrm{Cu}$ vacancies suppresses SDW order and enables superconductivity to emerge [67]. This scenario is supported by the observation that non-stoichiometric $\mathrm{CeCu}_{2} \mathrm{Si}_{2}$ samples exhibiting SDW order also exhibit pressure-induced superconductivity [68]. Therefore, $\mathrm{CeCu}_{2} \mathrm{Si}_{2}, \mathrm{CeCu}_{2} \mathrm{Ge}_{2}, \mathrm{CePd}_{2} \mathrm{Si}_{2}$, and $\mathrm{CeRh}_{2} \mathrm{Si}_{2}$ share a common predisposition for magnetic order that can be suppressed by applied pressure. The case of superconductivity in the heavy-fermion compound $\mathrm{CeNi}_{2} \mathrm{Ge}_{2}$ [69] does not exactly fit this pattern as we discuss below.

Bulk superconductivity was observed in the heavy-fermion compound $\mathrm{CeCu}_{2} \mathrm{Si}_{2}$ in 1979 at $T_{c}=0.6 \mathrm{~K}$ as confirmed by a specific heat jump of $\Delta C / \gamma T_{c}=1.4$ at $T_{c}(\gamma \simeq 1000 \mathrm{~mJ}$ $\mathrm{mol}^{-1} \mathrm{~K}^{-2}$ ) [1]. The SDW order observed in some samples occurs due to a Fermi-surface instability and is characterized by an incommensurate wave-vector $\vec{Q}=(0.215,0.215,0.530)$ with an ordered magnetic moment of $\sim 0.1 \mu_{B} / \mathrm{Ce}$ [70]. In such samples, superconductivity is induced by applying pressure or annealing the samples in $\mathrm{Cu}$ vapor. Measurements on single crystals of $\mathrm{CeCu}_{2} \mathrm{Si}_{2}$ reveal a modest anisotropy of the upper critical fields $H_{c 2}(T)$ when the magnetic field is aligned parallel and perpendicular to the Ce planes [66]. The initial slopes, $\left(d H_{c 2} / d T\right)_{T_{c}} \simeq-23 \mathrm{~T} / \mathrm{K}$, are isotropic but $H_{c 2}^{\perp}(0) \simeq 2.45 \mathrm{~T}$ and $H_{c 2}^{\|}(0) \simeq 2.05 \mathrm{~T}$ values are observed at zero temperature [66]. The Pauli paramagnetic limit for $\mathrm{CeCu}_{2} \mathrm{Si}_{2}$ is estimated [71] $H_{p 0}=1.84 T_{c} \simeq 1.2 \mathrm{~T}$ using $T_{c}=0.65 \mathrm{~K}$, and the orbital limiting field is calculated using WHH theory [59] to be $H_{c 2}^{*}(0)=0.693\left(-d H_{c 2} / d T\right)_{T_{c}} T_{c} \simeq 10.4 \mathrm{~T}$ using $\left(d H_{c 2} / d T\right)_{T_{c}} \simeq$ $-23 \mathrm{~T} / \mathrm{K}$. Given these estimates, it appears that superconductivity in $\mathrm{CeCu}_{2} \mathrm{Si}_{2}$ is paramagnetically-limited [72]; it is likely that $H_{c 2}(0)>H_{p 0}$ due to strong spin-orbit scattering, which is not accounted for in our estimate of $H_{p 0}$. The quadratic temperature dependence of the specific heat and thermal expansion coefficient [73] and nuclear quadrupole resonance measurements [74] in the superconducting state of $\mathrm{CeCu}_{2} \mathrm{Si}_{2}$ are consistent with the presence of line nodes [60] in the superconducting energy gap $\Delta(\vec{k})$. However, a recent study demonstrated that the specific heat deviates from a quadratic temperature dependence at very low-temperature [75]; this behavior is inconsistent with the presence of line nodes, but the specific heat data within the superconducting state can be accounted for by a scenario involving nodeless multiband superconductivity [75]. Furthermore, the absence of evidence for nodal quasiparticle excitations in the magnetic field dependence of $C$ at $60 \mathrm{mK}$ and the lack of resolvable oscillations of $C$ as a function of angle as the sample is rotated in a field within the $a b$ plane at $0.1 \mathrm{~K}$ and 0.2 $\mathrm{K}$ both cast doubt on the presence of line nodes in $\mathrm{CeCu}_{2} \mathrm{Si}_{2}$ [75]. These results may require us to reevaluate the conventional understanding about electron pairing in $\mathrm{CeCu}_{2} \mathrm{Si}_{2}$. When samples that exhibit superconductivity at ambient pressure are subjected to applied pressure, $T_{c}$ increases up to $\sim 4 \mathrm{GPa}$, and then decreases with increasing pressure [76]. Interestingly, a second dome of superconductivity is observed at higher pressure in slightly Ge-doped samples that is associated with a valence transition of the Ce ions (see Fig. 1) [77, 78]; this intriguing result suggests that superconductivity in $\mathrm{CeCu}_{2} \mathrm{Si}_{2}$ can be supported by both a magnetically-mediated pairing mechanism and an interaction based on spatially-extended density fluctuations.

A sinusoidally-modulated antiferromagnetic order below $T_{N}$ $=4.15 \mathrm{~K}$, characterized by incommensurate wave vector $\vec{Q}=$ $(0.284,0.284,0.543)$ and an ordered magnetic moment of 0.74 $\mu_{B} / \mathrm{Ce}$, is observed at ambient pressure in $\mathrm{CeCu}_{2} \mathrm{Ge}_{2}$ [79]. Following suppression of this antiferromagnetic order by applying pressure, superconductivity was observed in $\mathrm{CeCu}_{2} \mathrm{Ge}_{2}$ with $T_{c} \simeq 0.6 \mathrm{~K}$ near $8 \mathrm{GPa}$ [64]. The upper critical field was measured at an applied pressure of $\sim 10.1 \mathrm{GPa}$ and a value of $H_{c 2}(0) \sim 2 \mathrm{~T}$ was obtained with an initial slope of $\left(d H_{c 2} / d T\right)_{T_{c}} \simeq-11 \mathrm{~T} / \mathrm{K}$ [64]. The value of $H_{c 2}(0)$ is comparable to that of $\mathrm{CeCu}_{2} \mathrm{Si}_{2}$, and is also consistent with paramagnetic limiting $\left(H_{c 2}(0)<<H_{c 2}^{*}(0)\right)$. At ambient pressure and $\sim 1$ $\mathrm{GPa}$, specific heat measurements of $\mathrm{CeCu}_{2} \mathrm{Ge}_{2}$ in the antiferromagnetic state obtain $\gamma \simeq 200 \mathrm{~mJ} \mathrm{~mol}^{-1} \mathrm{~K}^{-2}$ [80]; however, measurements have not yet been performed in the superconducting state of $\mathrm{CeCu}_{2} \mathrm{Ge}_{2}$ to resolve a jump at $T_{c}$. These large values of $\gamma$ and $\left(d H_{c 2} / d T\right)_{T_{c}}$ are consistent with quasiparticles with enhanced masses and heavy-fermion superconductivity in $\mathrm{CeCu}_{2} \mathrm{Ge}_{2}$.

The compound $\mathrm{CePd}_{2} \mathrm{Si}_{2}$ is considered to be an intermedi- 


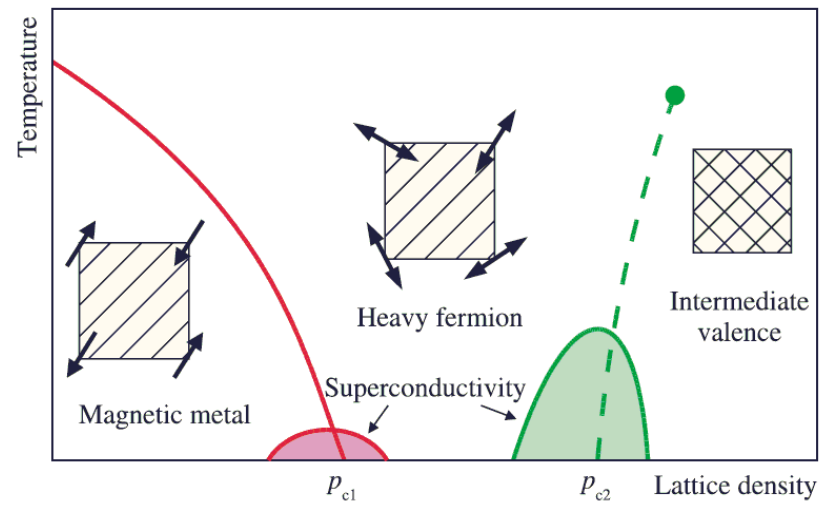

Figure 1: (Color online) Schematic phase diagram of temperature vs. lattice density for a generic Ce-based compound. At low density (lattice density typically tuned by pressure), stable localized magnetic moments participate in long-range antiferromagnetic order below a Néel temperature $T_{N}$. The red line represents the $T_{N}(P)$ phase boundary, which monotonically decreases towards a critical pressure $p_{c 1}$. Unconventional superconductivity, facilitated by magnetically-mediated pairing near $p_{c 1}$, is represented by the red dome, which screens or protects a quantum critical point. With increasing lattice density, the compound crosses over from heavy-fermion behavior to an intermediate valence region. This may proceed through a first-order phase transition (represented by the green dashed line) where a concomitant volume collapse of the unit cell takes place. When the critical end point where the first-order phase transition terminates (green filled circle) is at a sufficiently low temperature, superconductivity may form around the quantum phase transition associated with volume collapse at $p_{c 2}$ (the green dome). After Ref. [77].

ate valence system with antiferromagnetic order, characterized by wave vector $\vec{Q}=(0.5,0.5,0)[81]$, below $T_{N} \simeq 10 \mathrm{~K}$. This antiferromagnetic order is suppressed by applied pressure [82], and vanishes near $\sim 2.8 \mathrm{GPa}$ [57]. In the vicinity of this critical pressure, strong non-Fermi liquid behavior is observed, manifested by $\rho(T) \sim T^{1.2}$, and superconductivity with $T_{c}=0.43 \mathrm{~K}$ emerges (see inset of Fig. 2) [57, 58, 83]. Superconductivity appears as a dome in the temperature-pressure phase diagram centered around 2.8 GPa as is displayed in Fig. 2 [57, 58, 83]. The upper critical fields were measured at $2.67 \mathrm{GPa}$ in a single crystal of $\mathrm{CePd}_{2} \mathrm{Si}_{2}$ and found to be relatively anisotropic with values of $H_{c 2}^{a}(0) \simeq 0.7 \mathrm{~T}$ and $H_{c 2}^{c}(0) \simeq 1.3 \mathrm{~T}$ with large initial slopes of $\left(d H_{c 2}^{a} / d T\right)_{T_{c}} \simeq-12.7 \mathrm{~T} / \mathrm{K}$ and $\left(d H_{c 2}^{c} / d T\right)_{T_{c}} \simeq$ $-16 \mathrm{~T} / \mathrm{K}$ [83]. The Pauli paramagnetic limiting field can be estimated $H_{p 0} \simeq 0.8 \mathrm{~T}$, which is comparable to the observed $H_{c 2}(0)$ values; anisotropic paramagnetic limiting is a much more probable scenario [49] than orbital limiting since $H_{c 2}^{*}(0)$ values are estimated $H_{c 2}^{*, a}(0) \simeq 3.8 \mathrm{~T}$ and $H_{c 2}^{*, c}(0) \simeq 4.8 \mathrm{~T}$ using the $T_{c}$ and $\left(d H_{c 2} / d T\right)_{T_{c}}$ values. These large $\left(d H_{c 2} / d T\right)_{T_{c}}$ values, combined with a relatively large $\gamma=65(2) \mathrm{mJ} \mathrm{mol}^{-1} \mathrm{~K}^{-2}$ value at ambient pressure [84], imply that quasiparticle masses in $\mathrm{CePd}_{2} \mathrm{Si}_{2}$ are enhanced. This value of $\gamma$ was obtained by linearly extrapolating specific heat data, measured in the paramagnetic state and plotted as $C / T$ vs. $T^{2}$, to zero temperature [84]. Along with the case of $\mathrm{CeIn}_{3}$ (see Section 2.2), the heavy-fermion superconductivity exhibited by $\mathrm{CePd}_{2} \mathrm{Si}_{2}$ was invoked to argue strongly in favor of the existence of magnetically-mediated pairing in the vicinity of a magnetic quantum critical point [58].

The compound $\mathrm{CeRh}_{2} \mathrm{Si}_{2}$ exhibits antiferromagnetic order at

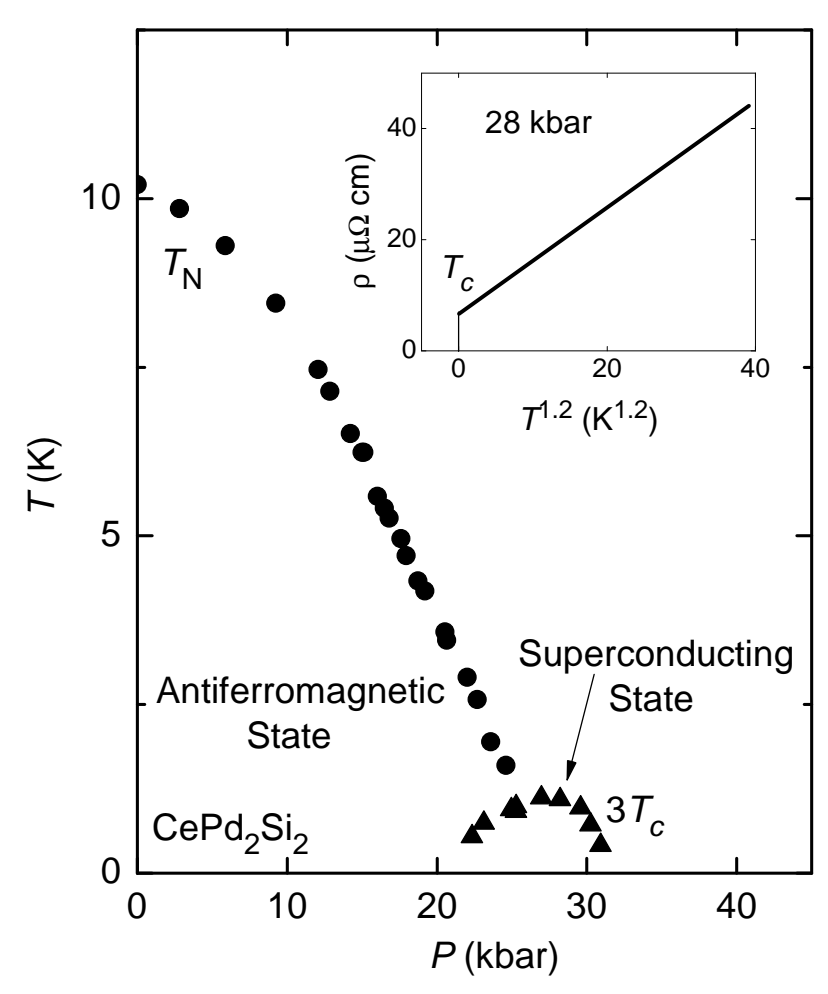

Figure 2: Temperature-pressure phase diagram determined for $\mathrm{CePd}_{2} \mathrm{Si}_{2}$. A dome of superconductivity is observed at low temperature $\left(T_{c}\right.$ values scaled by a factor of 3 for clarity) near the critical pressure where antiferromagnetic order, characterized by $T_{N}$, extrapolates to $0 \mathrm{~K}$. The inset displays electrical resistivity data $\rho(T)$ vs. $T^{1.2}$ at a pressure of $2.8 \mathrm{GPa}$; the data obey a nonFermi liquid $\rho(T) \propto T^{1.2}$ temperature dependence in the normal state. Adapted from Ref. [58].

ambient pressure below $T_{N, 1} \simeq 36 \mathrm{~K}$ [82]; this magnetic order is characterized by a wave vector $\vec{Q}=(1 / 2,1 / 2,0)$ and a large ordered magnetic moment of $1.34-1.42 \mu_{B} / \mathrm{Ce}$ [85]. Below $T_{N, 2} \simeq 24 \mathrm{~K}$, the magnetic structure changes so that it is described by two distinct wave vectors [85]. The low temperature properties of $\mathrm{CeRh}_{2} \mathrm{Si}_{2}$ are consistent with a Fermi liquid and the $\gamma \simeq 23 \mathrm{~mJ} \mathrm{~mol}^{-1} \mathrm{~K}^{-2}$ value, obtained from specific heat measurements within the antiferromagnetic state, is moderately enhanced [86]. Under applied pressure, $\gamma$ increases rapidly to $\gamma \sim 80 \mathrm{~mJ} \mathrm{~mol}^{-1} \mathrm{~K}^{-2}$ near $1.0 \mathrm{GPa}$, above which, it remains only weakly dependent on pressure [86]. Antiferromagnetic order is also suppressed by applied pressure and $T_{N, 1}$ and $T_{N, 2}$ vanish near $1.0 \mathrm{GPa}$ and $0.6 \mathrm{GPa}$, respectively [85]. Near 1.0 GPa, a narrow dome of superconductivity with $T_{c} \simeq 0.42 \mathrm{~K}$ emerges [65]. It is at this pressure that antiferromagnetic order terminates in a first-order phase transition as confirmed by a discontinuous volume change in measurements of thermal expansion under applied pressure [87]. The upper critical field for $\mathrm{CeRh}_{2} \mathrm{Si}_{2}$ has been measured at $\sim 1.1 \mathrm{GPa}$ on a polycrystalline sample to be $H_{c 2}(0) \simeq 0.28 \mathrm{~T}$ with an initial slope of $\left(d H_{c 2} / d T\right)_{T_{c}} \simeq-1 \mathrm{~T} / \mathrm{K}$ [65]. This value of $H_{c 2}(0)$ is very close to the estimated orbital-limiting field $H_{c 2}^{*}(0) \simeq 0.29$ $\mathrm{T}$ and much smaller than $H_{p 0} \simeq 0.8 \mathrm{~T}$. From the upper critical field, it has been estimated that the quasiparticles in $\mathrm{CeRh}_{2} \mathrm{Si}_{2}$ have enhanced effective masses of $m^{*} / m_{0} \simeq 200$ [65]. For addi- 
tional information, a description of the properties of the heavyfermion superconductor $\mathrm{CeRh}_{2} \mathrm{Si}_{2}$ can be found in Ref. [88].

The 1-2-2 compounds $\mathrm{CeCu}_{2} \mathrm{Si}_{2}, \mathrm{CeCu}_{2} \mathrm{Ge}_{2}, \mathrm{CePd}_{2} \mathrm{Si}_{2}$, and $\mathrm{CeRh}_{2} \mathrm{Si}_{2}$ all exhibit antiferromagnetic order that, when suppressed by applied pressure, makes way for heavy-fermion superconductivity to emerge; in contrast to this pattern, the situation for the isostructural compound $\mathrm{CeNi}_{2} \mathrm{Ge}_{2}$ is more complicated and its superconductivity is less well understood. At ambient pressure, $\mathrm{CeNi}_{2} \mathrm{Ge}_{2}$ exhibits spin fluctuations with a wave vector $\vec{Q}=(0.23,0.23,0.5)$ [89] that is comparable to the SDW order in other 1-2-2 compounds; however, long-range order does not develop in $\mathrm{CeNi}_{2} \mathrm{Ge}_{2}$ down to the lowest temperatures. The measured $\gamma \simeq 350 \mathrm{~mJ} \mathrm{~mol}^{-1} \mathrm{~K}^{-2}$ [90] definitely suggests that quasiparticles have enhanced effective masses; we note that $\gamma$ might even be significantly higher depending on how it is defined since $\mathrm{CeNi}_{2} \mathrm{Ge}_{2}$ exhibits non-Fermi liquid behavior at ambient pressure, manifested by $C / T \simeq-\ln (T)$ behavior at low temperature [91]. Traces of superconductivity were observed in $\mathrm{CeNi}_{2} \mathrm{Ge}_{2}$ below $0.1 \mathrm{~K}$, however, attempts to resolve a diamagnetic Meissner signal were unsuccessful [91]. In $\mathrm{CeNi}_{2} \mathrm{Ge}_{2}$ samples with extremely low disorder, complete superconducting transitions were observed resistively below $T_{c} \simeq$ $0.3 \mathrm{~K}$ [69]; however, whether this is bulk superconductivity remains an open question. Traces of superconductivity, presumably distinct from the ambient-pressure superconducting phase, were also observed in measurements of electrical resistivity under applied pressure near $1.5 \mathrm{GPa}$ with $T_{c}=0.22 \mathrm{~K}$ [92, 69]. The $T_{c}$ value associated with this superconducting state increases with increasing pressure up to $T_{c} \simeq 0.4 \mathrm{~K}$ at $2.6 \mathrm{GPa}$ [92]. If this superconductivity is intrinsic, it is possible that it could be related to a Ce valence instability as was observed in slightly $\mathrm{Ge}$-doped $\mathrm{CeCu}_{2} \mathrm{Si}_{2}$ samples (i.e., the phase diagram is similar to the one displayed in Fig. 1) [77, 78].

\section{2. $\mathrm{CeIn}_{3}, \mathrm{CeMIn} 5(\mathrm{M}=\mathrm{Co}, \mathrm{Rh}, \mathrm{Ir})$, and related compounds 2.2.1. Introduction}

The discovery [94] of pressure-induced superconductivity in the heavy-fermion antiferromagnet $\mathrm{CeRhIn}_{5}$ marked the beginning of subsequent discoveries of superconductivity in several related compounds. Each of these superconductors are members of a larger family of layered, tetragonal compounds with compositions $\mathrm{Ce}_{n} M_{m} \mathrm{In}_{3 n+2 m}$, where $M$ is a transition metal such as $\mathrm{Co}$, Rh, Ir, Pd and Pt, with the subset of $\mathrm{Ce} M \mathrm{In}_{5}$ materials being $n=1, m=1$ members with $M=\mathrm{Co}$, Rh, Ir. The basic structural building block of the family is the infinite layer, cubic compound $\mathrm{CeIn}_{3}$, which earlier had been found to become superconducting with applied pressure [58]. Figure 3 compares the pressure-dependent evolution of the Néel temperature $\left(T_{N}\right)$ and $T_{c}$ in $\mathrm{CeRhIn}$ 列 $\mathrm{CeIn}_{3}$. In both cases, a dome of superconductivity appears as $T_{N}$ is tuned toward $T=0 \mathrm{~K}$, but the maximum $T_{c}$ of CeRhIn 5 is more than an order of magnitude higher than that of $\mathrm{CeIn}_{3}$. This comparison suggests two significant conclusions: that fluctuations emerging from the $T=0 \mathrm{~K}$ magnetic quantum critical point may be responsible for Cooper pairing and that, as theoretically predicted [95], the quasi-twodimensional crystal structure and associated electronic structure of CeRhIn $n_{5}$ favors a higher $T_{c}$. Exploring the implications

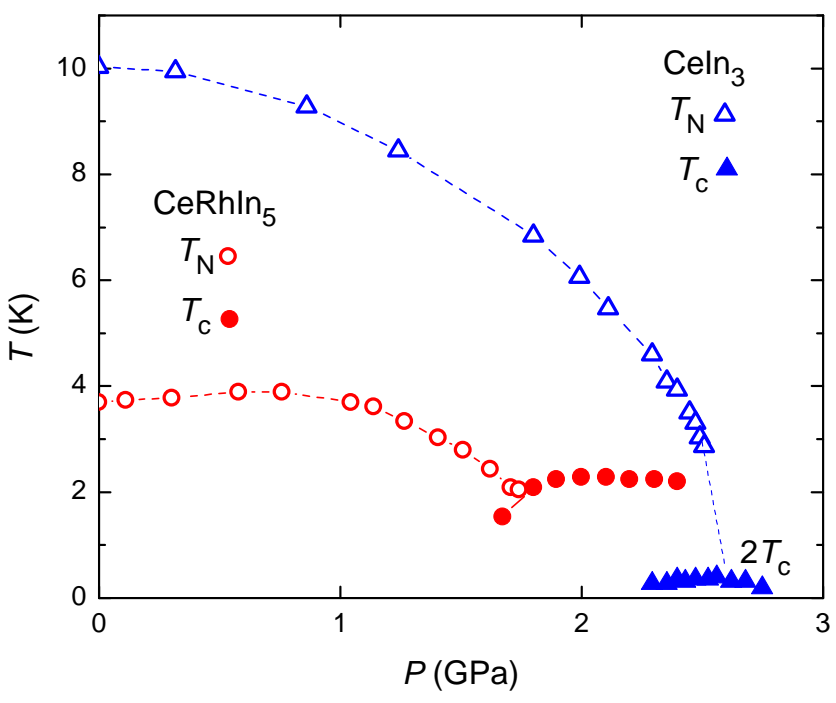

Figure 3: (Color online) Pressure dependence of $T_{N}$ and $T_{c}$ in CeRhIn 5 (unfilled and filled circles, respectively) and $\mathrm{CeIn}_{3}$ (unfilled and filled triangles, respectively). The phase diagrams for $\mathrm{CeIn}_{3}$ and $\mathrm{CeRhIn}_{5}$ were determined from electrical resistivity [58] and specific heat [93] measurements, respectively. Note that the $T_{c}$ values of $\mathrm{CeIn}_{3}$ are multiplied by a factor of 2 for clarity.

of these conclusions and, in particular, the relationship among magnetism, quantum criticality, and superconductivity has been a theme of subsequent studies of the $\mathrm{Ce}_{n} M_{m} \mathrm{In}_{3 n+2 m}$ superconductors.

Soon after the discovery of superconductivity in $\mathrm{CeRhIn}_{5}$, superconductivity was discovered at atmospheric pressure in $\mathrm{CeCoIn}_{5}$ and $\mathrm{CeIrIn}_{5}[96,97]$. These two new members were the first Ce-based heavy-fermion superconductors to be found at atmospheric pressure since the original discovery of $\mathrm{CeCu}_{2} \mathrm{Si}_{2}$ [1], and the $T_{c}$ of $2.3 \mathrm{~K}$ in $\mathrm{CeCoIn}_{5}$ remains the highest among Ce-based heavy-fermion superconductors at atmospheric pressure. $\mathrm{CeRhIn}_{5}, \mathrm{CeCoIn}_{5}$, and CeIrIn ${ }_{5}$ crystallize in the $\mathrm{HoCoGa}_{5}$ structure type $(P 4 / \mathrm{mmm})$, reported first by Grin et al. [98], and can be viewed as $n=1$ layers of CeIn ${ }_{3}$ separated by $m=1$ layers of ' $M \mathrm{In}_{2}$ ' that are stacked sequentially along the tetragonal $c$-axis. In addition to the $\mathrm{Ce} M \mathrm{In}_{5}$ materials, superconductivity is also found in compounds with $n=$ 1,2 or 3 and $m=1$ or 2 and in Pu-based analogs $\mathrm{Pu} M X_{5}$, where $M=\mathrm{Co}, \mathrm{Rh}$ and $X=\mathrm{In}$ or Ga. Table 1 summarizes the basic properties of superconductors in this broader family, all of which have been prepared in single crystal form and have large Sommerfeld coefficients. Non-magnetic variants of most of the Ce compounds have been prepared with La or Y and none is superconducting, which suggests that magnetism carried by the Ce ion is important for the appearance of superconductivity. Inspection of entries in this table also shows that the more structurally-anisotropic systems $(n=1)$ tend to have higher $T_{c}$ 's than those in which there are two $(n=2)$ or three $(n=3)$ adjacent $\mathrm{CeIn}_{3}$ units. This trend is consistent with the proposal that reduced dimensionality enhances the pairing interaction in spin-mediated superconductivity [95]. $\mathrm{Ce}_{2} \mathrm{RhIn}_{8}$ and CeIrIn are exceptions to this pattern, however, and we will return to them later. Of these systems, the $\mathrm{Ce} M \mathrm{In}_{5}(\mathrm{Ce} 115)$ materials 
have been studied most and are the primary topic to be discussed. The properties of the $\mathrm{Pu}$-analogs $\mathrm{Pu} M X_{5}$ are reviewed in a companion article in this issue of Physica $\mathrm{C}$ and are briefly touched upon in Section 4.3 of this article.

Band-structure calculations, confirmed through quantumoscillation measurements, find that the Ce115's are nearly compensated metals with a multi-sheeted electronic structure dominated by warped cylinders characteristic of quasi-twodimensional heavy band states [109, 110]. Though the effective magnetic moment above $\sim 200 \mathrm{~K}$ is close to the Hund's rule value for $\mathrm{Ce}^{+3}$, indicating that the $4 f$ electron of $\mathrm{Ce}$ is localized in each of the Ce115's at high temperatures [94, 96, 97], the electronic structure reveals a different perspective. De Haasvan Alphen (dHvA) measurements find a 'small' Fermi volume in CeRhIn 5 , comparable to that of $\mathrm{LaRhIn}_{5}$, which does not contain any $4 f$ electrons. On the other hand, the Fermi volume of $\mathrm{CeCoIn}_{5}$ and CeIrIn ${ }_{5}$ is 'large', implying that cerium's $4 f$ electron has become part of the Fermi sea in these compounds because of relatively stronger $f-p, d$ hybridization at low temperature $[109,110]$. Nevertheless, a two-dimensionallike Fermi surface topology is common to the Ce115's and would suggest that their physical properties should be relatively anisotropic. This is not the case. The normal state electrical resistivity $\rho$ and magnetic susceptibility $\chi$ of the Ce115's as well as their superconducting properties are only somewhat anisotropic, with $c$-axis $\rho$ and $\chi$ values about twice that of inplane values [111]. The upper critical magnetic fields are similarly anisotropic [111].

\subsubsection{Superconductivity}

Because of a relatively high $T_{c}$ at atmospheric pressure, superconductivity has been characterized most extensively in $\mathrm{CeCoIn}_{5}$. There is compelling evidence from several different types of experiments that the superconducting energy gap in $\mathrm{CeCoIn}_{5}$ has nodes and, more specifically, that the gap symmetry is $d_{x^{2}-y^{2}}$. For example, the thermal conductivity [112] and specific heat [113] exhibit a four-fold modulation as magnetic field is rotated in the tetragonal basal plane, consistent with the presence of line nodes in the gap. More direct probes of the superconducting gap by point-contact [114] and tunneling spectroscopies $[115,116]$ confirm with little doubt a gap with $d_{x^{2}-y^{2}}$ symmetry in $\mathrm{CeCoIn}_{5}$; this symmetry is also proven to exist in the high temperature cuprate superconductors from measurements sensitive to the phase of the order parameter [117]. Also like the cuprates [118], nodal superconductivity in $\mathrm{CeCoIn}_{5}$ develops out of an unusual normal state in which the electronic structure is partially gapped. First implied from electrical resistivity studies [119], this inference has been verified by tunneling spectroscopy $[115,120]$ in which a signature for a pseudogap-like structure appears at temperatures somewhat higher than $T_{c}$. Additionally, the four-fold modulation of thermal conductivity well below $T_{c}$ persists to temperatures at which the pseudogap emerges [112], suggesting that the pseudogap has the same nodal structure as the superconducting gap.

The presence of line nodes in the superconducting gap is reflected as well in power-law temperature dependencies of thermal conductivity $\left(\kappa \propto T^{3}\right.$ [121] or $\kappa \propto T^{2}$ [122]), spe- cific heat ( $\left.C \propto T^{2}[121]\right)$, and the nuclear spin-relaxation rate (1/ $T_{1} \propto T^{3+\epsilon}$ [123]) below $T_{c}$. The slightly greater than $T^{3}$ variation in $1 / T_{1}$ and different power laws for $\kappa$ may be a consequence of the multi-sheeted Fermi-surface topology of the Ce115's or the possibility that a small fraction of electrons at the Fermi surface does not participate in pairing, but these possibilities remain to be determined unambiguously. Nevertheless, in view of conclusions from tunneling spectroscopy, these power laws support the presence of line nodes in $\mathrm{CeCoIn}_{5}$ and also set a benchmark for interpreting similar power laws in CeIrIn 5 and $\mathrm{CeRhIn}_{5}$ [124]. As in CeCoIn 5 , there is a four-fold modulation in the specific heat of CeIrIn 5 [125, 126] and CeRhIn 5 [127] in their superconducting states, reinforcing the interpretation of gap nodes implied from power laws behaviors in them.

Table 2 summarizes the primary superconducting parameters of the Ce115's and includes, where available, corresponding properties of other family members based on Ce. A few comments about table entries are in order. The specific heat jump at $T_{c}$ and ratio of superconducting gap to $T_{c}$ are large in CeCoIn and in the purely superconducting state of CeRhIn 5 at high pressures; this implies strong electron-boson coupling. In contrast, $\Delta C / \gamma T_{c}$ is considerably smaller in other examples. At least in $\mathrm{Ce}_{2} \mathrm{CoIn}_{8}$, in which there are apparently two unidentified transitions above $T_{c}$ [128], and in $\mathrm{Ce}_{3} \mathrm{PdIn}_{11}$ with two magnetic transitions above $T_{c}$ [104], some entropy potentially available for superconductivity is removed by these transitions, which could be responsible in part for smaller jumps in specific heat. This effect is seen clearly in CeRhIn ${ }_{5}$ where the specific heat jump at $T_{c}$ is small in the pressure range with coexisting antiferromagnetic order and superconductivity but is large once magnetic order is suppressed completely [93]. CeIrIn 5 is a particularly interesting case. Its electrical resistivity drops to zero near $1.3 \mathrm{~K}$ with no clearly defined specific heat anomaly and bulk superconductivity only appears at $0.4 \mathrm{~K}$ [97]. Careful Hall effect and magnetoresistance measurements find evidence for a precursor state that extrapolates to $\sim 2 \mathrm{~K}$ in the zero-field limit [129] where there is also a change in slope of $C / T$ [97]. Conceivably, some entropy is associated with the precursor state that otherwise would be available for superconductivity. With applied pressure, the resistive transition to a $\rho=0 \mu \Omega \mathrm{cm}$ state in CeIrIn ${ }_{5}$ is essentially independent of pressure, but the bulk transition increases and eventually coincides with the resistive transition near $3 \mathrm{GPa}$, above which $T_{c}$ decreases with further increasing pressure [130]. This response suggests that some other state is competing with superconductivity as was found in $\mathrm{CeRhIn}_{5}$ as a function of pressure [131]. In this regard, we also note that no specific heat anomaly associated with bulk superconductivity has been found so far in $\mathrm{Ce}_{2} \mathrm{RhIn}_{8}$ at pressures up to $1.6 \mathrm{GPa}$ and temperatures above $0.35 \mathrm{~K}$ [132] even though $T_{N}$ extrapolates to $0 \mathrm{~K}$ near $2.5 \mathrm{GPa}$, which is close to where the resistive transition to $\rho=0 \mu \Omega \mathrm{cm}$ reaches $2 \mathrm{~K}$ [102]. This is unusual and deserves further exploration.

The initial slope of the upper critical magnetic field near $T_{c}$, $\left(d H_{c 2} / d T\right)_{T_{c}}$, is roughly twice as large for fields applied in the $a-b$ plane than perpendicular to it for $\mathrm{CeCoIn}_{5}$ and $\mathrm{CeIrIn}_{5}$ (for both its resistive and bulk transitions) and this is reflected as well in their $T=0 \mathrm{~K}$ upper critical fields $H_{c 2}(0)$. Inter- 
Table 1: Basic properties of members of the $\mathrm{Ce}_{n} M_{m} \mathrm{In}_{3 n+2 m}$ family as well as of the PuMX materials. The tabulated properties are at ambient pressure unless designated by pressure $(P)$ in units of GPa at which $T_{c}$ is a maximum. We define $\mathrm{SC}=$ superconductivity and AFM $=$ antiferromagnetism. The Sommerfeld coefficients are approximate, to order $\pm 10 \%$, in some cases because of a relatively strong temperature dependence of $C / T$ above a phase transition or because of a relative high $T_{c}$, e.g., in $\mathrm{Pu} M \mathrm{Ga}_{5}$ materials.

\begin{tabular}{lcccc}
\hline \hline Compound & Ground State & $T_{N}, T_{c}(\mathrm{~K})$ & $\gamma\left(\mathrm{mJ} \mathrm{mol}^{-1} \mathrm{~K}^{-2}\right)$ & References \\
\hline $\mathrm{CeCoIn}_{5}$ & $\mathrm{SC}$ & 2.3 & 250 & {$[96]$} \\
$\mathrm{CeRhIn}$ & $\mathrm{AFM} / \mathrm{SC}(\mathrm{P})$ & $3.8 / 2.4(2.3 \mathrm{GPa})$ & 430 & {$[94]$} \\
$\mathrm{CeIrIn}_{5}$ & $\mathrm{SC}$ & 0.4 & 750 & {$[97]$} \\
$\mathrm{CePt}_{2} \mathrm{In}_{7}$ & $\mathrm{AFM} / \mathrm{SC}(\mathrm{P})$ & $5.5 / 2.3(3.1 \mathrm{GPa})$ & 340 & {$[99]$} \\
$\mathrm{Ce}_{2} \mathrm{CoIn}_{8}$ & $\mathrm{SC}$ & 0.4 & 500 & {$[100]$} \\
$\mathrm{Ce}_{2} \mathrm{RhIn}_{8}$ & $\mathrm{AFM} / \mathrm{SC}(\mathrm{P})$ & $2.8 / 2.0(2.3 \mathrm{GPa})$ & 400 & {$[101,102]$} \\
$\mathrm{Ce}_{2} \mathrm{PdIn}_{8}$ & $\mathrm{SC}$ & 0.7 & 550 & {$[103]$} \\
$\mathrm{Ce}_{3} \mathrm{PdIn}_{11}$ & $\mathrm{AFM}+\mathrm{SC}$ & $1.6+1.5+0.4(\mathrm{SC})$ & 300 & {$[104]$} \\
$\mathrm{PuCoIn}_{5}$ & $\mathrm{SC}$ & 2.5 & 200 & {$[105]$} \\
$\mathrm{PuRhIn}_{5}$ & $\mathrm{SC}$ & 1.6 & 200 & {$[106]$} \\
$\mathrm{PuCoGa}_{5}$ & $\mathrm{SC}$ & 18.5 & 80 & {$[107]$} \\
$\mathrm{PuRhGa}$ & $\mathrm{SC}$ & 8.7 & $80-150$ & {$[108]$} \\
\hline \hline
\end{tabular}

Table 2: Superconducting parameters of the Ce115's and related materials. $\Delta C / \gamma T_{c}$ : jump in specific heat at $T_{c}$ divided by the Sommerfeld coefficient just above $T_{c} ; 2 \Delta / k_{B} T_{c}$ : twice the zero-temperature superconducting energy gap divided by $T_{c}$ (for CeRhIn 5 at $2.1 \mathrm{GPa}, T_{c}=2.2 \mathrm{~K}$ ); $\left(d H_{c 2} / d T\right)_{T_{c}}$ in units of T/K: slope of the upper critical field near $T_{c}$ for field applied in the plane $(a b)$ and perpendicular to it ( $c$-axis); $\xi(0)$ in units of $\AA$ : Ginzburg-Landau coherence length calculated from the measured zero-temperature upper critical field for field along the $c$-axis $\left(\xi_{a b}(0)\right)$ and in the basal plane $\left(\xi_{c}(0)\right)$; $\lambda$ in units of $\AA$ : magnetic penetration depth perpendicular $\left(\lambda_{a b}\right)$ and parallel to the $c$-axis $\left(\lambda_{c}\right)$. Because of the different $T_{c}$ 's determined by electrical resistance $(\sim 1.3 \mathrm{~K})$ and by a bulk measurement $(0.4 \mathrm{~K})$ through susceptibility $(\chi)$ or specific heat $(C)$, there are associated differences in $\left(d H_{c 2} / d T\right)_{T_{c}}$ and $\xi_{a b, c}(0)$ for CeIrIn ${ }_{5}$. References for each value are provided in

\begin{tabular}{|c|c|c|c|c|c|c|c|c|}
\hline$\overline{\text { Compound }}$ & $\Delta / \gamma T_{c}$ & $2 \Delta / k_{B} T_{c}$ & $d{ }^{2} H_{c 2}^{a b} / d T$ & $d H_{c 2}^{c} / d T$ & $\overline{\xi_{c}(0)}$ & $\overline{\xi_{a b}(0)}$ & $\overline{\lambda_{a b}}$ & $\overline{\lambda_{c}}$ \\
\hline \multirow[t]{2}{*}{$\mathrm{CeCoIn}_{5}$} & $4.5[96]$ & $10[123]$ & $-24[133]$ & $-11[133]$ & $53[134]$ & $82[96]$ & $1900[135]$ & $2700[135]$ \\
\hline & $4.7[133]$ & $6.05[136]$ & & $-8.2[121]$ & & $82[133,134]$ & 1900 [137] & 2800 [138] \\
\hline $\mathrm{CeRhIn}_{5}$ & $4.2[139]$ & $5[140]$ & $-15.5[141]$ & $-15.5[141]$ & $57[141]$ & $48[141]$ & & \\
\hline \multirow[t]{2}{*}{$\mathrm{CeIrIn}_{5}$} & $0.76[97]$ & $5.0[142]$ & $-4.8[97]$ & $-2.5[97]$ & $180[97,109]$ & 260 [109] & $6700[143]$ & \\
\hline & & $5[123]$ & $-10[97]$ & $-5[97]$ & $71[97]$ & 115 [109] & & \\
\hline $\mathrm{CePt}_{2} \mathrm{In}_{7}$ & $1.5[99]$ & & & -12 [99] & & & & \\
\hline $\mathrm{Ce}_{2} \mathrm{CoIn}_{8}$ & $0.2[128]$ & & & & $175[128]$ & 190 [128] & & \\
\hline $\mathrm{Ce}_{2} \mathrm{RhIn}_{8}$ & & & $-9.2[102]$ & & $77[102]$ & & & \\
\hline $\mathrm{Ce}_{2} \mathrm{PdIn}_{8}$ & $1.2[144]$ & & $-14[145]$ & $-14.3[144]$ & $130[144]$ & $120[144]$ & $1740[144]$ & \\
\hline $\mathrm{Ce}_{3} \mathrm{PdIn}_{11}$ & $0.6[104]$ & & $-9.6[104]$ & & & $120[104]$ & $9200[104]$ & \\
\hline
\end{tabular}




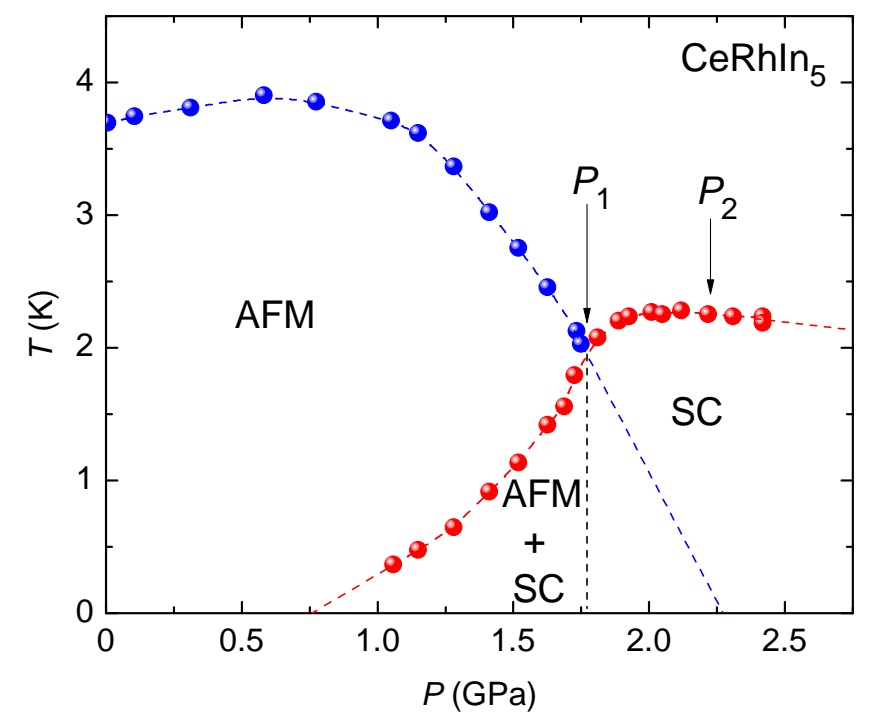

Figure 4: (Color online) Temperature versus pressure phase diagram of $\mathrm{CeRhIn}_{5}$ determined from specific heat measurements [93]. Regions of antiferromagnetic order and superconductivity are indicated by AFM and SC, respectively.

estingly, even though the Fermi-surface topology appears to be similar in $\mathrm{CeCoIn}_{5}$ and in $\mathrm{CeRhIn}_{5}$ at high pressures [146], this anisotropy is reversed in CeRhIn ${ }_{5}$ once magnetic order has been suppressed by pressure [141]. The origin of this anisotropy reversal is not currently understood and remains an important issue to be resolved. Below $0.3 T_{c}$, the upper critical field transition in $\mathrm{CeCoIn}_{5}$ is first order, consistent with strong Pauli limiting [147]. Although there is no clear evidence that the critical field transition becomes first order, it is also Pauli limited in CeRhIn 5 at high pressures [141] and in $\mathrm{Ce}_{2} \mathrm{PdIn}_{8}$ [144]. It also is noteworthy that $H_{c 2}(0)$ is nearly isotropic in the $n=2$, $m=1$ compounds $\mathrm{Ce}_{2} \mathrm{CoIn}_{8}$ and $\mathrm{Ce}_{2} \mathrm{PdIn}_{8}$, which might be expected because of their more three-dimensional crystal and, presumably, electronic structures. From this perspective, the upper critical fields of the $n=3, m=1$ material $\mathrm{Ce}_{3} \operatorname{PdIn}_{11}$ also should be nearly isotropic, but this remains to be seen.

\subsubsection{Magnetism, Quantum Criticality, and Superconductivity}

$\mathrm{CeRhIn}_{5}$ and $\mathrm{CeCoIn}_{5}$ exemplify the complex interplay among magnetism, quantum criticality, and superconductivity that makes them and other members of the $\mathrm{Ce}_{n} M_{m} \mathrm{In}_{3 n+2 m}$ family so fascinating. Figure 4 provides a detailed temperaturepressure phase diagram of CeRhIn $\mathrm{C}_{5}$. Below a critical pressure $P_{1}$, there is a range of pressures where incommensurate antiferromagnetic order coexists microscopically with superconductivity [148]. In this same pressure range, the ordered magnetic moment is only somewhat reduced relative to that expected of $\mathrm{Ce}^{3+}$ in a crystal-field doublet ground state $[149,150]$. A firstorder-like very small change in the $c$-axis incommensuration of the magnetic order appears where bulk superconductivity sets in at $1.5 \mathrm{GPa}$, signaling an interplay between the coexisting antiferromagnetic order and superconductivity [150]. Above $P_{1}$, there is no evidence for coexisting antiferromagnetism in zero applied field, but applying a magnetic field induces mag-

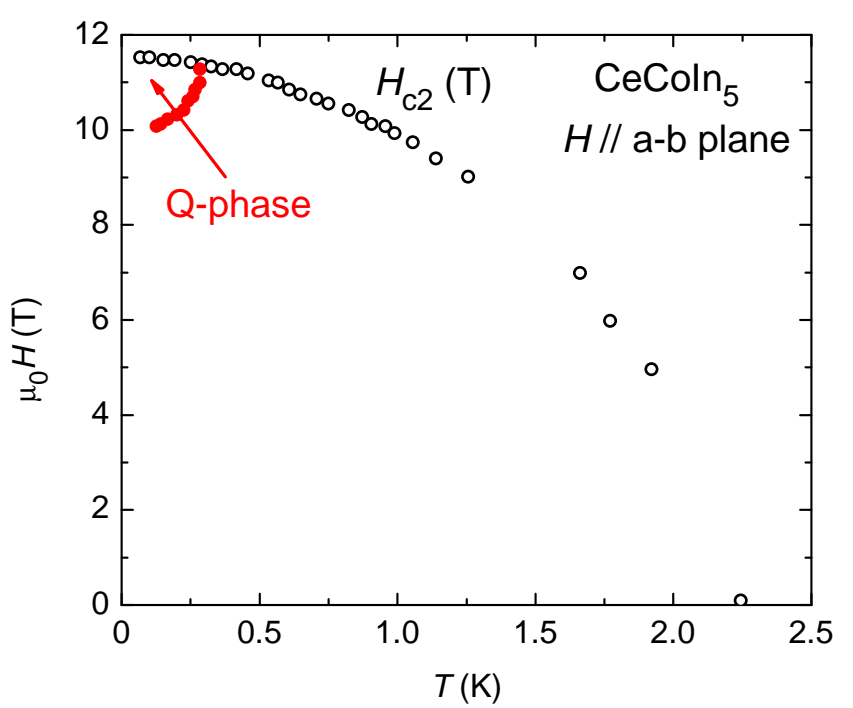

Figure 5: (Color online) Magnetic field-temperature phase diagram for $\mathrm{CeCoIn}_{5}$ determined for $H \| a-b$ plane. The Q-phase is bounded by the first-order upper critical field $H_{c 2}(T)$ and a line of second-order phase transitions indicated by solid circles. After Ref. [154].

netic order in the superconducting state [93, 151]. The applied magnetic field also allows the Néel boundary to be followed toward its $T=0 \mathrm{~K}$ quantum critical point ( $P_{2}$ in Fig. 4) near $2.3 \mathrm{GPa}$, where there is an abrupt increase in the dHvA frequencies to values that characterize the large-volume quasitwo-dimensional cyclotron orbits of $\mathrm{CeCoIn}_{5}$ [146]. Above this dome of unconventional superconductivity, the spin-relaxation rate follows a distinctly non-Fermi-liquid temperature dependence $\left(1 / T_{1} \propto T^{1 / 2}\right)$ [152], the resistivity just above $T_{c}$ increases by over a factor of 20 , and the temperature dependence of the electrical resistivity increases as $T^{0.85}$ [153]. Collectively, these responses suggest that the maximum $T_{c}$ in CeRhIn ${ }_{5}$ is associated with an unconventional type of quantum criticality in which magnetic as well as electronic degrees of freedom become critical at $P_{2}$ [153].

Unconventional superconductivity in $\mathrm{CeCoIn}_{5}$ develops from a non-Fermi-liquid normal state at atmospheric pressure [96, 119, 123]. Its dome-shaped $T_{c}(P)$ [119] suggests, like CeRhIn ${ }_{5}$, that antiferromagnetic order is 'nearby'. Indeed, replacing about $1 \%$ of its In atoms by $\mathrm{Cd}$ induces coexisting superconductivity and commensurate antiferromagnetism with a large ordered moment of $\sim 0.7 \mu_{B}[155,156,157]$. Applying a magnetic field to pure $\mathrm{CeCoIn}$, however, reveals evidence for a SDW-like quantum critical point near or just inside its $T=0 \mathrm{~K}$ upper critical field [158, 159, 160]. The Wiedemann-Franz law is violated near this quantum critical point for transport along the $c$ axis, but is obeyed for in-plane transport [161]. This result points to an anisotropic destruction of the Fermi surface of $\mathrm{CeCoIn}_{5}$ that is reminiscent of the pseudogap state in the underdoped cuprates and is possibly caused by spin fluctuations with a uniaxial character. Careful specific heat measurements reveal a phase transition just below $H_{c 2}(0)$ when the field is applied in the basal plane of $\mathrm{CeCoIn}_{5}$. This phase transition, first identified as a possible Fulde-Ferrell-Larkin-Ovchinnikov (FFLO) 
state [154], subsequently was shown by nuclear magnetic resonance (NMR) measurements to host antiferromagnetic order $[162,163]$. More recent neutron-diffraction experiments confirm field-induced incommensurate magnetic order with a small ordered moment of $0.15 \mu_{B}$ and further show that it is coupled to superconductivity and exists only in the so-called Q-phase illustrated in Fig. 5 [164]. Coupling of the SDW to $d$-wave superconductivity leads to a highly non-trivial state in which triplet $p$-wave superconductivity is induced [165]. The resulting spatially-modulated superconducting state is similar to an FFLO phase originally suggested from specific heat studies, but whether antiferromagnetism coexists with an FFLO state in the Q-phase remains to be determined. Applying pressure increases the $H-T$ phase space occupied by the Q-phase [166], which is surprising because pressure typically tends to suppress SDW order in Ce-based systems. In contrast, replacing only $0.05 \%$ of the In atoms by $\mathrm{Hg}$ wipes out any thermodynamic signature for it [167]. Besides a magnetic field, $\mathrm{Nd}$ substitution in $\mathrm{CeCoIn}_{5}$ induces magnetic order inside the zero-field superconducting state of $\mathrm{CeCoIn}_{5}$ for $\mathrm{Nd}$ concentrations less than 10\% [168]. Neutron diffraction measurements find that the Nd-induced antiferromagnetism has the same ordering wavevector and nearly the same small ordered moment as those in the Q-phase [169], raising the possibility that the two phenomena may be related.

Neodymium concentrations greater than $15 \%$ suppress superconductivity and, at this concentration, the electrical resistivity reaches $\sim 20 \mu \Omega \mathrm{cm}$ [168]. Interestingly, it is this value of the low-temperature electrical resistivity and not pair breaking by the magnetic moment of $\mathrm{Nd}$ that correlates with the suppression of superconductivity. As found in a systematic study of $T_{c}$ suppression by chemical substitution of Ce with various trivalent magnetic and non-magnetic lanthanides, $T_{c}$ reaches zero when the resistivity reaches $\sim 20 \mu \Omega \mathrm{cm}$, irrespective of the substituent $[170,168]$. This correlation suggests [170] an intimate relationship between the quantum criticality of $\mathrm{CeCoIn}_{5}$ and incoherent scattering produced by the introduction of Ce 'vacancies' that manifest themselves as Kondo-impurity scatterers in the superconducting state [171]. Ytterbium substitution for Ce in bulk single crystals of $\mathrm{Ce}_{1-x} \mathrm{Yb}_{x} \mathrm{CoIn}_{5}$ appeared to be a dramatic exception in that $T_{c}$ extrapolated to $0 \mathrm{~K}$ only in the limit $x \rightarrow 1[172,173]$; however, a study of $\mathrm{Ce}_{1-x} \mathrm{Yb}_{x} \mathrm{CoIn}_{5}$ thin films revealed a more rapid suppression rate of $T_{c}$ with $x$ such that superconductivity vanished near $x=0.4$ [174]. The discrepancy between these results was recently resolved via an unconventional application of Vegard's law in which it was determined that the actual $\mathrm{Yb}$ concentration $x_{a c t}$ in bulk single crystals is subnominal (i.e., $x_{\text {act }}<x$ ) [175]. Measurements of the $\mathrm{Yb}$ and Ce valences in $\mathrm{Ce}_{1-x} \mathrm{Yb}_{x} \mathrm{CoIn}_{5}[176,177]$ reveal that $\mathrm{Ce}$ is nearly trivalent for all $x$ and that the $\mathrm{Yb}$ valence decreases rapidly with increasing $x$ from $3+$ to a stable intermediate valence of $2.3+$ for $x \geq 0.2$ [177]. The measured unit-cell volume [172, 173], $V(x)$, is inconsistent with Vegard's law; however, the $\mathrm{Yb}$ concentration was adjusted in a plot of $V$ vs. $x$ until $V\left(x_{a c t}\right)$ was consistent with Vegard's law [175]. From this analysis, a correction function for the true $\mathrm{Yb}$ concentration, $x_{a c t}(x)$, was obtained that is consistent with independent measurements of the $\mathrm{Yb}$ concentration using wave- length dispersive spectroscopy, energy dispersive spectroscopy, and transmission X-ray absorption spectroscopy measurements $[175,173]$. It was found that $x_{a c t}=x / 3$ in bulk single crystals up to $x \sim 0.5$ [175]. The behavior of $T_{c}\left(x_{a c t}\right)$ for the bulk single crystals [175] is consistent with that of thin films of $\mathrm{Ce}_{1-x} \mathrm{Yb}_{x} \mathrm{CoIn}_{5}$ [174], resolving the discrepancy between their respective behaviors. Interestingly, superconductivity still persists to higher concentrations $[175,174]\left(x_{a c t} \simeq 0.4\right)$ than in cases where other other rare-earth ions are substituted for $\mathrm{Ce}$ $\left(x_{\text {act }} \sim 0.2\right)$ [170, 168, 178].

Motivated by the anomalous physical properties exhibited by $\mathrm{Ce}_{1-x} \mathrm{Yb}_{x} \mathrm{CoIn}_{5}[172,173,174]$, numerous studies have been performed to probe this system's superconducting and normal state properties $[179,180,181,182,183]$. Interestingly, near $x$ $=0.2\left(x_{a c t} \simeq 0.067\right)$, an intriguing crossover is observed. A valence transition occurs at this concentration where the $\mathrm{Yb}$ valence, which decreases rapidly from nearly $3+$ to $2.3+$ for $x_{a c t}<0.067$, maintains a stable intermediate valence of $2.3+$ for $x_{a c t} \geq 0.067$ [177]. This valence transition is concomitant with a reconstruction of the Fermi-surface topology wherein the heavy $\alpha$ sheets disappear and the Fermi surface resembles that of $\mathrm{YbCoIn}_{5}$ instead of $\mathrm{CeCoIn}_{5}$ [184]. Furthermore, from careful measurement of the in-plane magnetoresistivity of $\mathrm{Ce}_{1-x} \mathrm{Yb}_{x} \mathrm{CoIn}_{5}$ in magnetic fields up to $14 \mathrm{~T}$ [180], it has been shown that the quantum critical field associated with the fieldinduced quantum critical point $\left(H_{Q C P}=5 \mathrm{~T}\right.$ for $\left.\mathrm{CeCoIn} 5\right)$ is suppressed to $0 \mathrm{~T}$ near $x=0.2$; therefore, the quantum critical point is suppressed by $\mathrm{Yb}$ substitution and the non-Fermi liquid behavior that persists to much higher $\mathrm{Yb}$ concentrations in $\mathrm{Ce}_{1-x} \mathrm{Yb}_{x} \mathrm{CoIn}_{5}$ must be decoupled from its associated quantum criticality [180]. It has also been shown that quantum critical fluctuations near $x=0.2$ are suppressed with increasing pressure [183]. Despite all of these dramatic transitions and crossovers near $x=0.2$, there is no apparent feature in $T_{c}(x)$ at this concentration.

Recent measurements of the London penetration depth $\Delta \lambda$ have provided compelling evidence that the nodes, presumed to be associated with a $d_{x^{2}-y^{2}}$ symmetry of the superconducting energy gap, disappear near $x=0.2$ in $\mathrm{Ce}_{1-x} \mathrm{Yb}_{x} \mathrm{CoIn}_{5}$ [182]. Whereas substitution with $\mathrm{La}$ and $\mathrm{Nd}$ for $\mathrm{Ce}$ results in a powerlaw temperature dependence of $\Delta \lambda \sim T^{n}$ in which the exponent $n$ rapidly approaches $n=2$, indicating $d$-wave superconductivity in the dirty limit, $\mathrm{Yb}$ substitution for Ce results in an exponent of $n \geq 3$ (see Fig. 6) [182]. This observation implies that there is a topological transition of the superconducting energy gap symmetry in $\mathrm{Ce}_{1-x} \mathrm{Yb}_{x} \mathrm{CoIn}_{5}$ from nodal to nodeless near $x=0.2$ [182]. This surprising result might be explained by a composite pairing scenario in which a Lifshitz transition of the nodal Fermi surface is driven by $\mathrm{Yb}$ substitution, forming a fully-gapped $d$-wave molecular superfluid of composite pairs [185]. The $\Delta \lambda \sim T^{4}$ dependence of the penetration depth associated with the sound mode of this condensate agrees with the experimental results reported in Ref. [182].

If the composite pairing scenario for superconductivity in $\mathrm{Ce}_{1-x} \mathrm{Yb}_{x} \mathrm{CoIn}_{5}(x>0.2)$ applies, Ref. [185] predicts that the nodal behavior will return at a second quantum critical point at higher $\mathrm{Yb}$ concentration. The higher $\mathrm{Yb}$ concentration region 


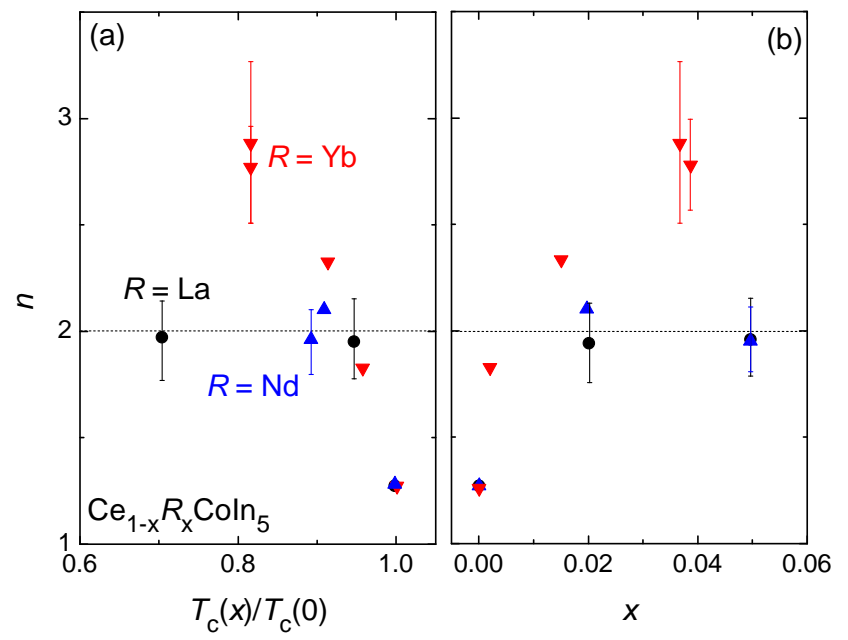

Figure 6: (Color online) Exponents $n$ for power-law behavior of lowtemperature London penetration depth $\Delta \lambda(T)$ data (see text) for the compounds $\mathrm{Ce}_{1-x} R_{x} \operatorname{CoIn}_{5}(R=\mathrm{La}, \mathrm{Nd}, \mathrm{Yb})$, plotted as a function of (a) $T_{c}(x) / T_{c}(0)$ and (b) $x$. Filled circles correspond to $R=\mathrm{La}$, filled upward-facing triangles correspond to $R=\mathrm{Nd}$, and filled downward-facing triangles correspond to $R=\mathrm{Yb}$. Dashed lines serve as guides to the eye emphasizing $n=2$, which is the expected exponent for a $d$-wave superconductor in the dirty limit. After Ref. [182].

of the phase diagram of $\mathrm{Ce}_{1-x} \mathrm{Yb}_{x} \mathrm{CoIn}_{5}$ is, therefore, currently being explored. The evolution of the many-body electronic state from a Kondo lattice consisting of Ce magnetic moments for low $\mathrm{Yb}$ concentration to a dilute array of Ce impurities at high $x$ has been studied [181]. A crossover was observed near $x=0.6$ between a regime characterized by localized Ce magnetic moments and one predominantly consisting of itinerant Yb electronic states [181]. Electronic correlations between the $\mathrm{Yb}$ ions can be inferred, and these $\mathrm{Yb}-\mathrm{Yb}$ correlations appear to be particularly strong for $0.65 \leq x \leq 0.775$ according to measurements of specific heat [181]. The search for a second quantum critical point as well as reconciling the notion of nodeless $d$-wave superconductivity with our understanding of heavyfermion superconductivity make the system $\mathrm{Ce}_{1-x} \mathrm{Yb}_{x} \mathrm{CoIn}_{5}$ a rich and intriguing playground within which to study the evolution and interplay of unconventional superconductivity, electronic structure, valence fluctuations, and quantum criticality.

The phase diagram in Fig. 7 of temperature versus $x$ in the Ce $M_{x} M_{1-x}^{\prime} \mathrm{In}_{5}$ series, where $M=\mathrm{Rh}$, Ir, Co, provides a broad perspective on the relationship between magnetism and superconductivity in the Ce115's [186]. In this figure, phase boundaries indicated by symbols are determined by specific heat for $\mathrm{CeRh}_{x} \mathrm{Co}_{1-x} \mathrm{In}_{5}$ [187] and $\mathrm{CeRh}_{1-x} \mathrm{Ir}_{x} \mathrm{In}_{5}$ [188]; whereas, dotted phase boundaries are deduced from neutron diffraction measurements on $\mathrm{CeRh}_{x} \mathrm{Co}_{1-x} \mathrm{In}_{5}$ [189] and $\mathrm{CeRh}_{1-x} \operatorname{Ir}_{x} \mathrm{In}_{5}$ [190]. With both Co and Ir substitution for Rh, superconductivity appears once the magnetic order is either commensurate (Co doping) or incommensurate coexisting with commensurate antiferromagnetism (Ir doping). In the case of Ir substitutions, nuclear quadrupole resonance experiments show that superconductivity coexists microscopically with magnetic order [191], and this is inferred as well from specific heat studies of Co-substituted crystals [187]. It seems, therefore, that commensurate order

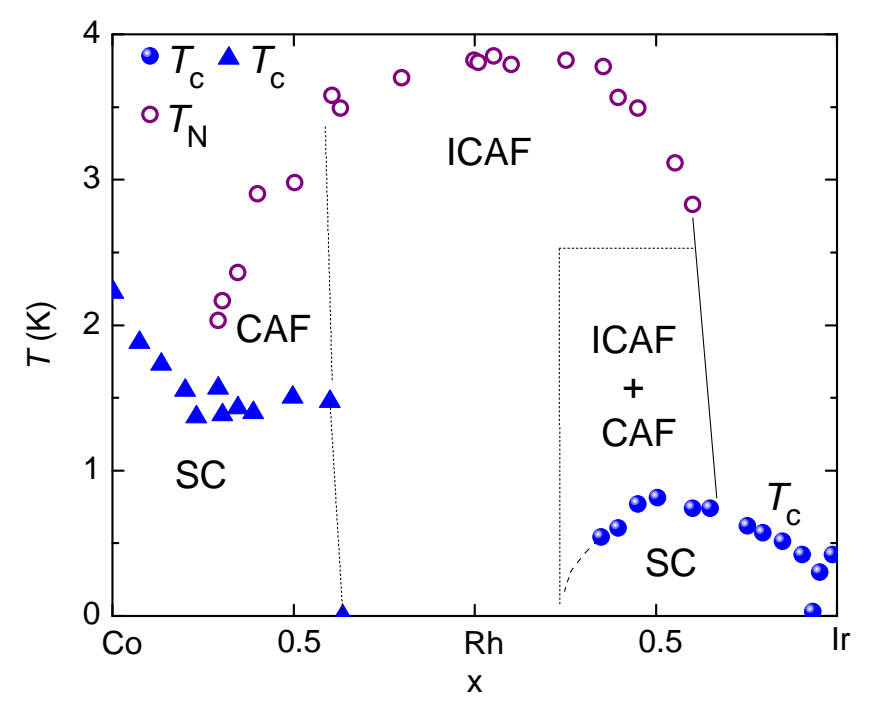

Figure 7: (Color online) Phase transitions in $\mathrm{Ce} M_{x} M_{1-x}^{\prime} \mathrm{In}_{5}$ compounds as $M$ $=\mathrm{Rh}$ is replaced by $M^{\prime}=\mathrm{Ir}$ and Co. In this figure, we define ICAF = incommensurate antiferromagnetism; $\mathrm{CAF}=$ commensurate antiferromagnetism; SC = superconductivity. After Ref. [186].

allows or favors the development of $d$-wave superconductivity in these systems, which appears as well to be the case in $\mathrm{CePt}_{2} \mathrm{In}_{7}$ [192]. Though there are important commonalities in the relationship between superconductivity and magnetism across the series, there are also significant differences. With Ir substitution, a dome of superconductivity appears that is centered somewhat below the critical Ir content at which the Néel boundary extrapolates to $T=0 \mathrm{~K}$. Near $90 \% \mathrm{Ir}, T_{c}$ goes to zero and this notch in $T_{c}(x)$ widens with applied pressure, while $T_{c}$ of CeIrIn ${ }_{5}$ increases and the $T_{c}$ of samples with lower Ir content grows toward $T_{c} \sim 2 \mathrm{~K}$; this value is comparable to that of pure CeRhIn ${ }_{5}$ under pressure [193]. This response to pressure suggests that there may be two superconducting phases in the $\mathrm{CeRh}_{1-x} \mathrm{Ir}_{x} \mathrm{In}_{5}$ series. On the other hand, with increasing Co content, superconductivity appears suddenly at a Co concentration of $\sim 40 \%$, and again, applied pressure enhances $T_{c}$ toward 2 K [194]. The rapid onset of superconductivity at ambient pressure appears to be associated with a jump in the cyclotron frequency of at least one heavy-mass sheet of the Fermi surface to a value typical of the large Fermi volume of pure $\mathrm{CeCoIn}_{5}$ [195]. Though it seems likely that the broad dome of superconductivity in Ir-doped CeRhIn ${ }_{5}$ may be associated with a magnetic quantum critical point, this is not the case with Co-doping where dHvA measurements do not find a diverging mass near $40 \%$ Co [195].

\subsubsection{Superconducting Mechanism and Broader Relationships}

Without a phase-sensitive measurement, it is not possible to prove that superconductivity in the Ce115's is unconventional. Nevertheless, all measurements consistently point to the conclusion that it is and that the pairing has a nodal, spin-singlet character. With superconductivity appearing in proximity to antiferromagnetism in these as well as other members of the broader family and the development of superconductivity out of 
a non-Fermi-liquid normal state, it is reasonable to expect that fluctuations of a quantum-critical nature play a dominant role in producing Cooper pairing [196]. In $\mathrm{CeCoIn}_{5}$, this criticality is of the conventional Hertz-Millis-Moriya type that involves only critical fluctuations of a magnetic order parameter [158], which favors pairing in the $d$-wave channel. In contrast, the critical response of CeRhIn 5 under pressure is consistent with criticality of magnetic as well as electronic degrees of freedom [127]. Such an unconventional type of quantum criticality also favors $d$-wave pairing [197]. Though Fig. 7 implies that superconductivity in CeIrIn 5 is separated from magnetism, NMR and specific heat measurements argue that $\operatorname{CeIrIn}_{5}$ is close to a $T=$ $0 \mathrm{~K}$ instability of a SDW at ambient pressure [198, 199], which again would favor magnetically-mediated $d$-wave pairing.

A neutron-spin resonance develops in the superconducting state of $\mathrm{CeCoIn}_{5}$ [200], with the ratio of resonance energy to superconducting gap being the same as in the cuprates [201]. Further searches for a neutron resonance in CeRhIn under pressure and in CeIrIn ${ }_{5}$ will be challenging, but worthwhile. Besides the spin resonance, results of scanning tunneling spectroscopy [202, 115] strongly support magnetic pairing in $\mathrm{CeCoIn}_{5}$. Remarkably, this magnetically-mediated superconductivity persists in thin films that are just a few monolayers thick [203]. Magnetically-mediated $d$-wave superconductivity and the presence of a pseudogap and spin resonance as well as a non-Fermi-liquid normal state above $T_{c}$ in the Ce115's are strikingly similar to the high- $T_{c}$ cuprates. Though the absolute value of $T_{c}$ is much lower in the Ce115's than in the cuprates, relative to the electronic energy/magnetic scale relevant for superconductivity, the $T_{c}$ 's of the Ce115's are just as high as those in the cuprates [204]. The ease with which superconductivity in the Ce115's can be tuned and with which they can be grown as very high quality crystals, in addition to the perspective provided by dimensional tuning in the broader $\mathrm{Ce}_{n} M_{m} \mathrm{In}_{3 n+2 m}$ family, make these materials particularly useful for unraveling commonalities in heavy-fermion superconductors and unconventional superconductors more broadly.

\subsection{Noncentrosymmetric Ce-based compounds}

Superconductivity in Ce-based heavy-fermion compounds with noncentrosymmetric crystal structures is an exciting, relatively new area of research. Much of the interest in this topic is driven by the rather unique possibility of superconductivity exhibiting an unconventional mixture of $s-$ and $p$-wave pairing that is allowed when inversion symmetry is absent. This fascinating and rich subject has been reviewed recently by Kimura and Bonalde in Ref. [205] and is also the subject of a review article by F. Kneidinger et al. in this special issue of Physica C. Therefore, we will only touch briefly on the properties of four Ce-based heavy-fermion compounds with noncentrosymmetric crystal structures: the compound $\mathrm{CePt}_{3} \mathrm{Si}$ exhibits superconductivity at ambient pressure [206] while pressure-induced superconductivity is observed in $\mathrm{CeRhSi}_{3}, \mathrm{CeIrSi}_{3}$, and $\mathrm{CeCoGe}_{3}$ [207, 208, 209].

The crystal structure of $\mathrm{CePt}_{3} \mathrm{Si}$ is described by tetragonal space group $P 4 m m$ which lacks inversion symmetry [206]. Long-range antiferromagnetic order is observed below $T_{N}=2.2$
$\mathrm{K}$ with superconductivity emerging near $T_{c}=0.75 \mathrm{~K}$ at ambient pressure [206]. As is discussed in Ref. [49], some samples of $\mathrm{CePt}_{3} \mathrm{Si}$ exhibit $T_{c} \simeq 0.45 \mathrm{~K}$ with sharper phase transitions. A Sommerfeld coefficient, $\gamma=390 \mathrm{~mJ} \mathrm{~mol}^{-1} \mathrm{~K}^{-2}$, is measured which is consistent with heavy-fermion behavior [206]. Furthermore, a jump in the specific heat of $\Delta C / \gamma T_{c} \simeq$ 0.25 is observed at $T_{c}$, confirming that superconductivity is bulk in $\mathrm{CePt}_{3} \mathrm{Si}$ [206]. The upper critical fields were found to be $H_{c 2}(0) \sim 5 \mathrm{~T}$ at zero temperature with an initial slope of $\left(d H_{c 2} / d T\right)_{T_{c}} \simeq-8.5 \mathrm{~T} / \mathrm{K}$ from measurements of polycrystalline samples [206]. This value of $H_{c 2}(0)$ is comparable to the estimated [59] orbital limiting field $H_{c 2}^{*}(0) \simeq 4.4 \mathrm{~T}$ and significantly exceeds the estimated [71] Pauli limiting field of $H_{p 0} \simeq 1.4 \mathrm{~T}$. Since $H_{c 2}(0)>>H_{p 0}$, we expect that pairing in $\mathrm{CePt}_{3} \mathrm{Si}$ is unconventional and involves spin-triplet pairs. Upper critical field measurements on a single crystal of $\mathrm{CePt}_{3} \mathrm{Si}$ with $T_{c}=0.63 \mathrm{~K}$ demonstrated very little anisotropy in $H_{c 2}(T)$ curves and the extrapolated $H_{c 2}(0)$ values also exceeded the Pauli paramagnetic limit [210]. For more information concerning the early work on $\mathrm{CePt}_{3} \mathrm{Si}$, see Ref. [211].

Pressure-induced superconductivity was observed in the compounds $\mathrm{CeRhSi}_{3}$ [207], $\mathrm{CeIrSi}_{3}$ [208], and $\mathrm{CeCoGe}_{3}$ [209] that all form in a noncentrosymmetric crystal structure characterized by the tetragonal space group $14 \mathrm{~mm}$. In each case, antiferromagnetic order is suppressed by applied pressure, enabling superconductivity to emerge. Enhanced Sommerfeld coefficient values of $\gamma \simeq 120 \mathrm{~mJ} \mathrm{~mol}^{-1} \mathrm{~K}^{-2}$ [207], $120 \mathrm{~mJ} \mathrm{~mol}^{-1}$ $\mathrm{K}^{-2}$ [208], and $32 \mathrm{~mJ} \mathrm{~mol}^{-1} \mathrm{~K}^{-2}$ [212] for $\mathrm{CeRhSi}_{3}, \mathrm{CeIrSi}_{3}$, and $\mathrm{CeCoGe}_{3}$, respectively, are observed at ambient pressure. These values suggest that the effective quasiparticle masses are enhanced in these compounds. At ambient pressure, the magnetic ordering temperatures are $T_{N} \simeq 1.6 \mathrm{~K}$ and $T_{N} \simeq 5 \mathrm{~K}$ for $\mathrm{CeRhSi}_{3}$ and $\mathrm{CeIrSi}_{3}[207,208]$, respectively, while $\mathrm{CeCoGe}_{3}$ exhibits three successive transitions at $T_{N 1} \simeq 21 \mathrm{~K}, T_{N 2} \simeq 12$ $\mathrm{K}$, and $T_{N 3} \simeq 8 \mathrm{~K}$ [209]. Under applied pressure, antiferromagnetic order is suppressed in each compound and superconductivity emerges. For $\mathrm{CeRhSi}_{3}$, superconductivity is observed at pressures above $\sim 1.2 \mathrm{GPa}$ and exists in a wide dome up to 3 GPa with maximum $T_{c} \simeq 1 \mathrm{~K}$ near $2.3 \mathrm{GPa}$ [207]; the lowerpressure side of the dome appears to coexist with antiferromagnetic order [207]. For $\mathrm{CeIrSi}_{3}$, antiferromagnetic order vanishes for pressures near $2.25 \mathrm{GPa}$, and a dome of superconductivity is observed with maximum $T_{c} \simeq 1.65 \mathrm{~K}$ near $2.6 \mathrm{GPa}$ [208]. Finally, applied pressure suppresses antiferromagnetic order in $\mathrm{CeCoGe}_{3}$ with $T_{N 1}$ (the highest magnetic transition temperature) vanishing near 5.5 GPa [209]. Superconductivity is observed in a broad dome centered near 6.5 GPa with maximum $T_{c} \simeq 0.69 \mathrm{~K}$ [209]. The upper critical fields of these compounds are all enormous and significantly exceed their respective Pauli paramagnetic limits. Initial slopes of $\left(d H_{c 2} / d T\right)_{T_{c}} \simeq-23 \mathrm{~T} / \mathrm{K}$ (2.6 GPa) and $-20 \mathrm{~T} / \mathrm{K}$ (at $6.5 \mathrm{GPa}$ ) for $\mathrm{CeRhSi}_{3}$ [213] and $\mathrm{CeCoGe}_{3}$ [212], respectively, suggest that $H_{c 2}(0)$ values may exceed $30 \mathrm{~T}$ for these compounds when $H \| c$ ! For $\mathrm{CeIrSi}_{3}$, the upper critical field values at $2.65 \mathrm{GPa}$ and zero temperature are $H_{c 2}^{a}(0) \simeq 9.5 \mathrm{~T}$ and $H_{c 2}^{c}(0) \approx 45 \mathrm{~T}$ with initial slopes of $\left(d H_{c 2}^{a} / d T\right)_{T_{c}} \simeq-14.5 \mathrm{~T} / \mathrm{K}$ and $\left(d H_{c 2}^{c} / d T\right)_{T_{c}} \simeq-17 \mathrm{~T} / \mathrm{K}$ (as seen in Fig. 8) [214]. These values of $H_{c 2}(0)$ are also consistent with 


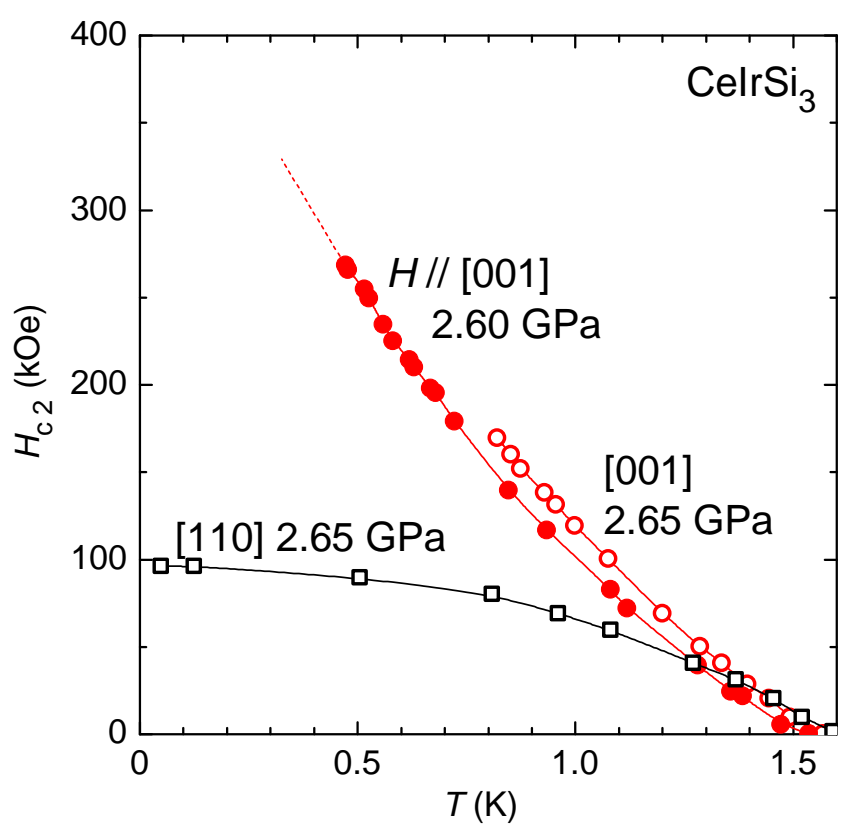

Figure 8: (Color online) The anisotropic upper critical fields $H_{c 2}(T)$ for $\mathrm{CeIrSi}_{3}$. The magnetic field $H$ is applied along the $c$-axis under applied pressures of $2.60 \mathrm{GPa}$ (filled circles) and $2.65 \mathrm{GPa}$ (unfilled circles), and along [110] under an applied pressure of $2.65 \mathrm{GPa}$ (unfilled squares). The dramatic anisotropy in $H_{c 2}(T)$ is apparent and the $H_{c 2}^{c}(0)$ value appears to be close to 40 $\mathrm{T}$ at 2.60 GPa (after Ref. [214]).

spin-triplet pairing and attest to the unconventional nature of pressure-induced superconductivity in these noncentrosymmetric heavy-fermion compounds.

\section{4. $\mathrm{CeNiGe}_{3}, \mathrm{Ce}_{2} \mathrm{Ni}_{3} \mathrm{Ge}_{5}$, and $\mathrm{CePd} \mathrm{Al}_{5}$}

We conclude our discussion of Ce-based heavy-fermion superconducters by discussing three compounds that do not fall neatly into the categories we have just discussed. This includes $\mathrm{CeNiGe}_{3}, \mathrm{Ce}_{2} \mathrm{Ni}_{3} \mathrm{Ge}_{5}$, and $\mathrm{CePd}_{5} \mathrm{Al}_{2}$, and the properties of each compound are briefly addressed below.

Though it has the same 1-1-3 stoichiometry as many of the noncentrosymmetric superconductors discussed in Section 2.3, the compound $\mathrm{CeNiGe}_{3}$ crystallizes with the centrosymmetric orthorhombic space group Cmmm and exhibits antiferromagnetic order below $T_{N} \simeq 5.5 \mathrm{~K}$ at ambient pressure [215]. In an analysis of specific heat data that includes fitting a Schottkyanomaly feature, the Sommerfeld coefficient was reported to be $\gamma \simeq 45 \mathrm{~mJ} \mathrm{~mol}^{-1} \mathrm{~K}^{-2}$ [215]. Analysis of Curie-Weiss behavior in the magnetic susceptibility of $\mathrm{CeNiGe}_{3}$ provides a $\mu_{\text {eff }}$ value that is very close to the expected value for a localized magnetic moment associated with $\mathrm{Ce}^{3+}$ ions [215]. Under applied pressure, $T_{N}$ initially increases up to about $\sim 3 \mathrm{GPa}$ and then rapidly decreases, vanishing near $\sim 5.5 \mathrm{GPa}$ [216]; an incomplete superconducting transition was first reported in $\rho(T)$ measurements of $\mathrm{CeNiGe}_{3}$, and a dome of superconductivity, centered near this critical pressure with maximum $T_{c} \simeq 0.48$ $\mathrm{K}$ as defined by the transition's onset, was obtained in the temperature-pressure phase diagram [216]. Measurements of $\mathrm{CeNiGe}_{3}$ under pressure were later conducted with improved pressure homogeneity, obtaining qualitatively different results [217]. In the study of Kotegawa et al., superconductivity first emerges near $1.7 \mathrm{GPa}$ (coexisting with the antiferromagnetic phase of $\mathrm{CeNiGe}_{3}$ ) and traces out two distinct superconducting domes with maximum $T_{c}$ values of $0.32 \mathrm{~K}$ and $0.43 \mathrm{~K}$ near pressures of $\sim 3.5 \mathrm{GPa}$ and $\sim 6.8 \mathrm{GPa}$, respectively [217]; this higher pressure coincides with the suppression of $T_{N}$ to $0 \mathrm{~K}$ [217]. The observation of a diamagnetic signal in the magnetic susceptibility suggests that the superconductivity associated with the lower pressure dome could be bulk. In contrast, the superconducting transition within the higher pressure dome becomes incomplete in measurements of electrical resistivity conducted in modest magnetic fields [217]; this observation suggests that the higher pressure phase might not be bulk superconductivity [217]. The upper critical field of $\mathrm{CeNiGe}_{3}$ at zero temperature was found to increase significantly under applied pressure from $H_{c 2}(0) \simeq 0.02 \mathrm{~T}$ at $1.9 \mathrm{GPa}$ to $H_{c 2}(0) \simeq 1.56$ $\mathrm{T}$ at $6.8 \mathrm{GPa}$ [217]. The initial slope of the upper critical field experiences a comparable increase from $\left(d H_{c 2} / d T\right)_{T_{c}} \simeq-0.24$ $\mathrm{T} / \mathrm{K}$ at $1.9 \mathrm{GPa}$ to $\left(d H_{c 2} / d T\right)_{T_{c}} \simeq-10.8 \mathrm{~T} / \mathrm{K}$ at $6.8 \mathrm{GPa}$ [217]. Below $3.4 \mathrm{GPa}$, superconductivity is orbitally-limited, but it is probably paramagnetically-limited in the higher pressure dome [217]. Though the question of whether superconductivity at higher pressure is bulk remains an outstanding issue, the large observed value of $\left(d H_{c 2} / d T\right)_{T_{c}} \simeq-10.8 \mathrm{~T} / \mathrm{K}$ at $6.8 \mathrm{GPa}$ and the appearance of superconductivity in the vicinity of a suppressed antiferromagnetic phase are consistent with the usual signatures of heavy-fermion superconductivity.

The compound $\mathrm{Ce}_{2} \mathrm{Ni}_{3} \mathrm{Ge}_{5}$ crystallizes with orthorhombic space group Ibam [218]. A Sommerfeld coefficient of $\gamma \simeq$ $90 \mathrm{~mJ}$ mol-Ce $\mathrm{C}^{-1} \mathrm{~K}^{-2}$ is obtained [219], and antiferromagnetic order, characterized by phase transitions at $T_{N, 1}=5.1 \mathrm{~K}$ and $T_{N, 2}=4.5 \mathrm{~K}$, is exhibited by $\mathrm{Ce}_{2} \mathrm{Ni}_{3} \mathrm{Ge}_{5}[219,218]$. The magnetic entropy recovered at $T_{N, 1}$ is $S_{m a g} \simeq 0.67 R \ln 2$, which is reduced slightly from the expected $S_{\text {mag }}$ for a doublet ground state because of the Kondo effect [219]. A neutron diffraction study on a polycrystalline sample of $\mathrm{Ce}_{2} \mathrm{Ni}_{3} \mathrm{Ge}_{5}$ was only able to resolve the transition at $T_{N, 1}$ and obtained ordered moments of $0.4(1) \mu_{B} / \mathrm{Ce}$ at $1.4 \mathrm{~K}$, polarized antiparallel to one another along the $a$ axis [218]. Under applied pressure, $T_{N, 1}$ is suppressed, vanishing completely near $3.9 \mathrm{GPa}$; it was not possible to track $T_{N, 2}$ as a function of applied pressure due to broadening of the feature associated with this transition [220]. Near $3.9 \mathrm{GPa}$, a dome of superconductivity is observed with maximum $T_{c} \simeq 0.26 \mathrm{~K}$ (defined by the onset) [220]. At $3.6 \mathrm{GPa}$, an upper critical field of $H_{c 2}(0) \simeq 0.7 \mathrm{~T}$ is measured at $0 \mathrm{~K}$ and the initial slope is $\left(d H_{c 2} / d T\right)_{T_{c}} \simeq-8 \mathrm{~T} / \mathrm{K}$ [220]. This value of $H_{c 2}(0)$ slightly exceeds the Pauli paramagnetic limiting field, estimated to be $H_{p 0} \simeq 0.48 \mathrm{~T}$, but is roughly a factor of 2 smaller than the estimate for $H_{c 2}^{*}(0) \simeq 1.4 \mathrm{~T}$. This result implies that superconductivity in $\mathrm{Ce}_{2} \mathrm{Ni}_{3} \mathrm{Ge}_{5}$ is paramagnetically-limited, and the enhanced values of $\gamma$ and $\left(d H_{c 2} / d T\right)_{T_{c}}$ suggest that heavy quasiparticles participate.

The final compound we will discuss in this section is $\mathrm{CePd}_{5} \mathrm{Al}_{2}$. Following the discovery of superconductivity in the isostructural compound $\mathrm{NpPd}_{5} \mathrm{Al}_{2}$ [221] (see Section 4.3), pressure-induced superconductivity was observed in this heavy- 
fermion compound. In $\mathrm{CePd}_{5} \mathrm{Al}_{2}$, two distinct antiferromagnetic states are observed at ambient pressure that are characterized by $T_{N, 1}=3.9 \mathrm{~K}$ and $T_{N, 2}=2.9 \mathrm{~K}$ [222]. The Sommerfeld coefficient value for $\mathrm{CePd}_{5} \mathrm{Al}_{2}$ is $\gamma \simeq 56 \mathrm{~mJ} \mathrm{~mol}^{-1} \mathrm{~K}^{-2}$ at ambient pressure [222], which is consistent with modestlyenhanced quasiparticle masses. Under applied pressure, $T_{N, 1}$ first increases and then decreases with increasing pressure up to $\sim 8-9 \mathrm{GPa}$ [222]; in the vicinity of this critical pressure, a dome of superconductivity is observed. The maximum $T_{c} \simeq 0.57 \mathrm{~K}$ is observed near $10.8 \mathrm{GPa}$ and measurements of the upper critical field values at this pressure with $H \| c$ yield $H_{c 2}^{c}(0) \simeq 0.25 \mathrm{~T}$ with initial slope $\left(d H_{c 2}^{c} / d T\right)_{T_{c}} \simeq-1.04 \mathrm{~T} / \mathrm{K}$ [222]. This value of $H_{c 2}(0)$ is roughly a factor of 2 smaller than the orbital limit, estimated to be $H_{c 2}^{*, c}(0) \simeq 0.41 \mathrm{~T}$ using the values of $T_{c}$ and $\left(d H_{c 2}^{c} / d T\right)_{T_{c}}$. On the other hand, $H_{c 2}(0)<<H_{p 0} \simeq 1 \mathrm{~T}$, so further investigations will be necessary to identify the primary pair-breaking mechanism for superconductivity in $\mathrm{CePd}_{5} \mathrm{Al}_{2}$ in a magnetic field.

\section{U-based heavy-fermion superconductors}

\subsection{Introduction}

Following the discovery of heavy-fermion superconductivity in $\mathrm{CeCu}_{2} \mathrm{Si}_{2}$ in 1979 [1], four superconducting uranium compounds with large $\gamma$ values were identified in the time frame 1983-84: $\mathrm{UBe}_{13}\left(T_{c}=0.9 \mathrm{~K}, \gamma=1.0 \mathrm{~J}\right.$ mol-U $\left.\mathrm{U}^{-1} \mathrm{~K}^{-2}\right)$ [52], $\mathrm{U}_{6} \mathrm{Fe}\left(T_{c}=4.0 \mathrm{~K}, \gamma=24 \mathrm{~mJ}\right.$ mol-U $\left.{ }^{-1} \mathrm{~K}^{-2}\right)$ [225], $\mathrm{UPt}_{3}\left(T_{c}\right.$ $\left.=0.54 \mathrm{~K}, \gamma=450 \mathrm{~mJ} \mathrm{~mol}-\mathrm{U}^{-1} \mathrm{~K}^{-2}\right)$ [53], and $\mathrm{U}_{2} \mathrm{PtC}_{2}\left(T_{c}\right.$ $=1.5 \mathrm{~K}, \gamma=75 \mathrm{~mJ}$ mol- $\mathrm{U}^{-1} \mathrm{~K}^{-2}$ ) [228]. These observations quickly demonstrated that heavy-fermion behavior is a general phenomenon that is not restricted to Ce-based compounds and generated an enormous amount of interest in the physics of heavy-fermion materials. Interestingly, Bucher et al. had already reported superconductivity in $\mathrm{UBe}_{13}$ [50]; however, the superconductivity was attributed to filaments of uranium since it persisted in high magnetic fields. Specific heat measurements by Ott et al. showed that $\mathrm{UBe}_{13}$ displayed bulk superconductivity with an enormous value of $\gamma$ [52] comparable with that of $\mathrm{CeCu}_{2} \mathrm{Si}_{2}$. The compound $\mathrm{U}_{6} \mathrm{Fe}$ was already known to exhibit superconductivity according to the work of Chandrasekar and Hulm [265]. Magnetoresistance measurements by DeLong et al. demonstrated that $\mathrm{U}_{6} \mathrm{Fe}$ has a rather large upper critical field $H_{c 2}(T)$ whose magnitude is consistent with its value of $\gamma$ [225]. The bulk superconductivity of $\mathrm{UPt}_{3}$ observed by Stewart et al. [53] had not previously been reported.

The compounds $\mathrm{UBe}_{13}$ and $\mathrm{UPt}_{3}$ both exhibit unconventional superconductivity in which the pairing of electrons is widely believed to be mediated by magnetic interactions rather than the electron-phonon interaction. The observation of superconducting properties with power-law temperature dependencies provides evidence for anisotropic superconductivity in which the electrons are paired in states with angular momentum greater than zero and the energy gap vanishes at points or on lines on the Fermi surface. An example of the extraordinary properties of the superconducting state in these materials is the upper critical field of $\mathrm{UBe}_{13}$. There is also evidence for multiple superconducting phases, presumably with different order parameter symmetries, in $\mathrm{U}_{1-x} \mathrm{Th}_{x} \mathrm{Be}_{13}$ and $\mathrm{UPt}_{3}$ under pressure or in an applied magnetic field [266, 267, 268, 269].

\subsection{Superconductivity coexisting with or on the border of an- tiferromagnetic order}

\subsection{1. $U M_{2} A l_{3}(M=N i, P d)$}

Coexistence between superconductivity and antiferromagnetic order was discovered in the heavy-fermion compounds $\mathrm{UPd}_{2} \mathrm{Al}_{3}$ and $\mathrm{UNi}_{2} \mathrm{Al}_{3}$ in 1991 [236, 241]. The critical temperatures of $T_{c}=2 \mathrm{~K}$ and $T_{N}=14 \mathrm{~K}$ for $\mathrm{UPd}_{2} \mathrm{Al}_{3}$ and $T_{c}=1.06 \mathrm{~K}$ and $T_{N}=4.6 \mathrm{~K}$ for $\mathrm{UNi}_{2} \mathrm{Al}_{3}$ are well-separated with $T_{c}<T_{N}$ [236, 241]. Each compound forms with the same hexagonal crystal structure described by space group $P 6 / \mathrm{mmm}$. The U-U separations of $4.186 \AA$ and $4.018 \AA$ for $\mathrm{UPd}_{2} \mathrm{Al}_{3}$ and $\mathrm{UNi}_{2} \mathrm{Al}_{3}$, respectively, are well above the Hill limit [270] $(\sim 3.4 \AA)$, indicating that the $5 f$ electron states from $\mathrm{U}^{4+}$ ions do not overlap significantly, and are expected to exhibit a strongly-localized character. However, hybridization with itinerant electron states leads to some amount of itineracy of the $5 f$ electrons, the degree of which is still a matter of debate for these compounds [49]. The splitting of the $\mathrm{U}^{4+} J=4$ multiplet by the CEF has been studied using magnetic susceptibility measurements [271, 272]. Though the splitting energies are different, the energy level schemes are identical for both compounds; each has a $\Gamma_{4}$ singlet ground state with excited states, listed in order of increasing energy, of a $\Gamma_{1}$ singlet, $\Gamma_{6}$ doublet, $\Gamma_{5}$ doublet, $\Gamma_{3}$ singlet, and $\Gamma_{5}$ doublet. The splitting between the ground and first excited states is $33 \mathrm{~K}$ for $\mathrm{UPd}_{2} \mathrm{Ni}_{3}$ and $100 \mathrm{~K}$ for $\mathrm{UNi}_{2} \mathrm{Al}_{3}$, coinciding with local maxima in the magnetic susceptibility data near $35 \mathrm{~K}$ and $100 \mathrm{~K}$, respectively [271, 272].

Commensurate antiferromagnetic order at $T_{N}=14 \mathrm{~K}$ (in zero magnetic field) is observed in $\mathrm{UPd}_{2} \mathrm{Al}_{3}$, characterized by a wave vector $\vec{Q}=(0,0,1 / 2)$ and an ordered magnetic moment of $0.85 \mu_{B} / \mathrm{U}$ [273]. The magnetic entropy released at $T_{N}$ is $S_{m a g}$ $=0.65 R \ln 2$ [237]. In applied magnetic fields, several distinct magnetic phases emerge that are discussed in detail in Ref. [49]. In contrast, the more itinerant character of the $5 f$ electron states in $\mathrm{UNi}_{2} \mathrm{Al}_{3}$ results in an incommensurate SDW order at $T_{N}=$ 4.6 $\mathrm{K}$, characterized by a wave vector $\vec{Q}=(1 / 2 \pm \delta, 0,1 / 2)$ where $\delta=0.110(3)$ and a small ordered magnetic moment of $0.24(10) \mu_{B} / \mathrm{U}[274,275]$. This subtle phase transition produces no feature in the electrical resistivity [276] and was missed entirely in the first neutron-diffraction study of $\mathrm{UNi}_{2} \mathrm{Al}_{3}$ [273]. An entropy of $S_{m a g}=0.12 R \ln 2$ is released at $T_{N}[241,277]$ that is consistent with the smaller ordered magnetic moment in $\mathrm{UNi}_{2} \mathrm{Al}_{3}$.

At low temperature, strong electronic correlations lead to enhanced values of the Sommerfeld coefficient. For $\mathrm{UPd}_{2} \mathrm{Al}_{3}, \gamma$ $=210 \mathrm{~mJ} \mathrm{~mol}^{-1} \mathrm{~K}^{-2}$ is measured in the paramagnetic state and $\gamma=150 \mathrm{~mJ} \mathrm{~mol}{ }^{-1} \mathrm{~K}^{-2}$ is obtained in the temperature range $T_{c}<T<T_{N}$ [236]. These values are comparable to $\gamma=120$ $\mathrm{mJ} \mathrm{mol}^{-1} \mathrm{~K}^{-2}$ obtained for $\mathrm{UNi}_{2} \mathrm{Al}_{3}$ in its normal state [241]. Using a simple single-band model and making other approximations, the enhanced masses of the quasiparticles were estimated to be $m^{*} / m_{0} \sim 66$ for $\mathrm{UPd}_{2} \mathrm{Al}_{3}$ and $\sim 70$ for $\mathrm{UNi}_{2} \mathrm{Al}_{3}$ [236, 241]. 
Table 3: Summary of properties of heavy-fermion superconductors including: space group of crystal structure; superconducting critical temperature $T_{c}$ (asterisk indicates superconductivity induced by applied pressure); Néel $\left(T_{N}\right)$, Curie $\left(T_{C}\right)$, quadrupolar ordering $\left(T_{Q}\right)$, or hidden ordering temperature at ambient pressure; Sommerfeld coefficient $\gamma$ (reported in units of $\mathrm{J} \mathrm{mol}^{-1} \mathrm{~K}^{-2}$ ); jump in specific heat at $T_{c}, \Delta C / \gamma T_{c}$; type of nodes in the superconducting energy gap; upper critical field values at zero temperature $H_{c 2}(0)$ (reported in units of T); and references from which tabulated results were obtained. When $H_{c 2}(0)$ values were only available from studies on a polycrystalline sample, the value is denoted with $\dagger$.

\begin{tabular}{|c|c|c|c|c|c|c|c|c|c|}
\hline Compound & Structure & $T_{c}(\mathrm{~K})$ & $T_{N}, T_{C}, T_{Q}(\mathrm{~K})$ & $\gamma$ & $\Delta C / \gamma T_{c}$ & Nodes & $H_{c 2}^{a b}(0)$ & $H_{c 2}^{c}(0)$ & References \\
\hline $\mathrm{CeCu}_{2} \mathrm{Si}_{2}$ & $I 4 / \mathrm{mmm}$ & $0.6-0.7$ & 0.9 & 1 & 1.4 & Line & 2.05 & 2.45 & {$[1,66,73,74]$} \\
\hline $\mathrm{CeCu}_{2} \mathrm{Ge}_{2}$ & $I 4 / \mathrm{mmm}$ & $0.64^{*}$ & 4.15 & 0.2 & & & $2^{\dagger}$ & & {$[79,64,80]$} \\
\hline $\mathrm{CePd}_{2} \mathrm{Si}_{2}$ & $I 4 / \mathrm{mmm}$ & $0.43^{*}$ & 10 & 0.065 & & & 0.7 & 1.3 & {$[57,58,83,84]$} \\
\hline $\mathrm{CeRh}_{2} \mathrm{Si}_{2}$ & $I 4 / \mathrm{mmm}$ & $0.42^{*}$ & 36,24 & 0.023 & & & $0.28^{\dagger}$ & & {$[82,85,86,65]$} \\
\hline $\mathrm{CeNi}_{2} \mathrm{Ge}_{2}$ & $I 4 / \mathrm{mmm}$ & 0.3 & & 0.35 & & & & & {$[90,69]$} \\
\hline $\mathrm{CeIn}_{3}$ & $P m 3 m$ & $0.19^{*}$ & 10.2 & 0.14 & & Line & 0.45 & 0.45 & {$[58,223]$} \\
\hline $\mathrm{CeCoIn}_{5}$ & $P 4 / \mathrm{mmm}$ & 2.3 & & 0.25 & 4.5 & Line & 11.6 & 4.95 & {$[96,133]$} \\
\hline $\mathrm{CeRhIn}_{5}$ & $P 4 / \mathrm{mmm}$ & $2.4^{*}$ & 3.8 & 0.43 & 4.2 & & 9.7 & 16.9 & {$[94,139,141]$} \\
\hline $\mathrm{CeIrIn}_{5}$ & $P 4 / \mathrm{mmm}$ & 0.4 & & 0.75 & 0.76 & & 1.0 & 0.5 & [97] \\
\hline $\mathrm{CePt}_{3} \mathrm{Si}$ & $P 4 m m$ & 0.75 & 2.2 & 0.39 & 0.25 & Line & 2.7 & 3.2 & {$[206,210]$} \\
\hline $\mathrm{CeIrSi}_{3}$ & $I 4 m m$ & $1.65^{*}$ & 5 & 0.12 & & & 9.5 & 45 & {$[208,214]$} \\
\hline $\mathrm{CeRhSi}_{3}$ & $I 4 m m$ & $1^{*}$ & 1.6 & 0.12 & & & 7 & $>30$ & {$[207,213]$} \\
\hline $\mathrm{CeCoGe}_{3}$ & $I 4 m m$ & $0.69^{*}$ & $21,12,8$ & 0.032 & & & & $>30$ & {$[212,209]$} \\
\hline $\mathrm{CeNiGe}_{3}$ & Cmmm & $0.43^{*}$ & 5.5 & 0.045 & & & 1.56 & & {$[215,216,217]$} \\
\hline $\mathrm{Ce}_{2} \mathrm{Ni}_{3} \mathrm{Ge}_{5}$ & Ibam & $0.26^{*}$ & $5.1,4.5$ & 0.09 & & & 0.7 & & {$[218,219,220]$} \\
\hline $\mathrm{CePd}_{5} \mathrm{Al}_{2}$ & $I 4 / \mathrm{mmm}$ & $0.57^{*}$ & $3.9,2.9$ & 0.056 & & & & 0.25 & [222] \\
\hline $\mathrm{U}_{6} \mathrm{Fe}$ & $\mathrm{I} 4 / \mathrm{mcm}$ & 3.8 & & 0.157 & 2.1 & & $10^{*}$ & & {$[224,225,226]$} \\
\hline $\mathrm{U}_{2} \mathrm{PtC}_{2}$ & $\mathrm{I} 4 / \mathrm{mmm}$ & 1.47 & & 0.15 & & & 7.8 & 9.2 & {$[227,228,229]$} \\
\hline $\mathrm{UBe}_{13}$ & $F m 3 c$ & 0.95 & & 1 & 2.5 & Line & 14 & & {$[52,230]$} \\
\hline $\mathrm{UPt}_{3}$ & $\mathrm{~Pb}_{3} / m m c$ & $0.53,0.48$ & 5 & 0.44 & $0.55,0.27$ & Line, Point & 2.1 & 2.8 & {$[53,231,232,233,234$} \\
\hline $\mathrm{URu}_{2} \mathrm{Si}_{2}$ & $I 4 / \mathrm{mmm}$ & 1.53 & 17.5 & 0.07 & 0.93 & Line & 3 & 14 & {$[55,54,235]$} \\
\hline $\mathrm{UPd}_{2} \mathrm{Al}_{3}$ & $P 6 / \mathrm{mmm}$ & 2.0 & 14 & 0.21 & 1.48 & Line & 3.3 & 3.9 & {$[236,237,238,239,240$} \\
\hline $\mathrm{UNi}_{2} \mathrm{Al}_{3}$ & $P 6 / \mathrm{mmm}$ & 1.06 & 4.6 & 0.12 & 0.4 & & 0.9 & 0.35 & {$[241,242]$} \\
\hline $\mathrm{UGe}_{2}$ & Cmmm & $0.8^{*}$ & 52 & $0.110^{*}$ & $0.2-0.3$ & Line & & & {$[243,244,245,246]$} \\
\hline URhGe & Pnma & 0.25 & 9.5 & 0.164 & 0.45 & & $2,1.3$ & 0.55 & {$[247,248]$} \\
\hline $\mathrm{UCoGe}$ & Pnma & 0.6 & 2.5 & 0.057 & 1 & Point & 5 & 0.5 & {$[249,250,251,252,253$} \\
\hline UIr & $P 2_{1}$ & $0.15^{*}$ & 46 & 0.049 & & & & & {$[254,255]$} \\
\hline $\mathrm{PrOs}_{4} \mathrm{Sb}_{12}$ & $\operatorname{Im} \overline{\mathbf{3}}$ & 1.85 & & 0.5 & 3 & Point & 2.3 & & {$[32,256,257,258]$} \\
\hline $\operatorname{PrTi}_{2} \mathrm{Al}_{20}$ & $F d \overline{3} m$ & $0.2,1.1^{*}$ & 2.0 & 0.1 & & & $>3^{*}$ & & {$[259,260,31]$} \\
\hline $\operatorname{PrV}_{2} \mathrm{Al}_{20}$ & $F d \overline{3} m$ & 0.05 & 0.6 & 0.09 & 0.3 & & 0.014 & & {$[259,62]$} \\
\hline$\beta-\mathrm{YbAlB}_{4}$ & Cmmm & 0.08 & & 0.15 & & & 0.15 & 0.025 & {$[63,261]$} \\
\hline $\mathrm{PuCoGa}_{5}$ & $\mathrm{P} 4 / \mathrm{mmm}$ & 18.5 & & 0.077 & 1.4 & Line & & & {$[107,262,263]$} \\
\hline $\mathrm{PuRhGa}_{5}$ & $P 4 / \mathrm{mmm}$ & 8.7 & & 0.07 & 0.65 & Line & 27 & 15 & {$[108,262,264]$} \\
\hline $\mathrm{NpPd}_{5} \mathrm{Al}_{2}$ & $I 4 / \mathrm{mmm}$ & 4.9 & & 0.2 & 2.33 & Point & 3.7 & 14 & [221] \\
\hline
\end{tabular}


Superconductivity occurs at $T_{c}=2 \mathrm{~K}$ and $T_{c}=1.06 \mathrm{~K}$ in $\mathrm{UPd}_{2} \mathrm{Al}_{3}$ and $\mathrm{UNi}_{2} \mathrm{Al}_{3}$, respectively, and corresponding jumps in the specific heat $\Delta C / \gamma T_{c}$ of 1.48 and 0.4 are observed at their respective $T_{c}$ 's $[236,237,241]$. The response of superconductivity in these compounds to applied pressure differ. In $\mathrm{UPd}_{2} \mathrm{Al}_{3}, T_{c}$ remains nearly unchanged for applied pressures up to $6.5 \mathrm{GPa}$ and is suppressed linearly for $P>6.5 \mathrm{GPa}$ with a gradual rate of $d T_{c} / d P \sim-0.05 \mathrm{~K} / \mathrm{GPa}$ [278]. In contrast, the superconducting state of $\mathrm{UNi}_{2} \mathrm{Al}_{3}$ is suppressed at a relatively rapid rate of $d T_{c} / d P \sim-0.24(3) \mathrm{K} / \mathrm{GPa}$ under low applied pressures [279]. The upper critical fields $H_{c 2}(T)$ have been measured at ambient pressure in experiments on single crystals with the field applied parallel and perpendicular to the basal plane (parallel to $a$ axis). Zero-temperature values for $\mathrm{UPd}_{2} \mathrm{Al}_{3}$ were determined to be $H_{c 2}^{a}(0)=3.3 \mathrm{~T}$ and $H_{c 2}^{c}(0)=3.9 \mathrm{~T}$ with initial slopes of $\left(d H_{c 2}^{a} / d T\right)_{T_{c}} \simeq-4.6 \mathrm{~T} / \mathrm{K}$ and $\left(d H_{c 2}^{c} / d T\right)_{T_{c}} \simeq-5.45$ $\mathrm{T} / \mathrm{K}$ [238, 242]. These $H_{c 2}$ values are close to the Pauli paramagnetic limiting field of $H_{p 0}=3.7 \mathrm{~T}$ and much smaller than values for the orbital critical fields, estimated using the $\mathrm{WHH}$ theory [59] to be $H_{c 2}^{*, a}(0)=6.4 \mathrm{~T}$ and $H_{c 2}^{*, c}(0)=7.6 \mathrm{~T}$. Therefore, the relatively isotropic upper critical fields for $\mathrm{UPd}_{2} \mathrm{Al}_{3}$ are paramagnetically-limited. This result can be contrasted with the observation of significant anisotropy in $H_{c 2}(T)$ for $\mathrm{UNi}_{2} \mathrm{Al}_{3}$, which exhibits orbital limiting [242]. The upper critical fields were determined to be $H_{c 2}^{a}(0) \approx 0.9 \mathrm{~T}$ and $H_{c 2}^{c}(0) \approx 0.35 \mathrm{~T}$ with initial slopes of $\left(d H_{c 2}^{a} / d T\right)_{T_{c}} \simeq-1.14 \mathrm{~T} / \mathrm{K}$ and $\left(d H_{c 2}^{c} / d T\right)_{T_{c}} \simeq$ $-0.42 \mathrm{~T} / \mathrm{K}$ [242]. When these are compared with estimated values for the paramagnetic limiting field, $H_{p 0}=0.18 \mathrm{~T}$, and the orbital critical fields, $H_{c 2}^{*, a}(0)=0.79 \mathrm{~T}$ and $H_{c 2}^{*, c}(0)=0.29 \mathrm{~T}$, the $H_{c 2}(0)$ values for $\mathrm{UNi}_{2} \mathrm{Al}_{3}$ suggest that orbital limiting is the dominant pair-breaking mechanism in a magnetic field. Since $H_{p 0}<H_{c 2}(0) \sim H_{c 2}^{*}(0)$, spin-triplet pairing may be present in $\mathrm{UNi}_{2} \mathrm{Al}_{3}$. This possibility was also suggested in an early study of $H_{c 2}(T)$ curves on polycrystalline samples [276] and ${ }^{27} \mathrm{Al}$ Knight shift measurements provide additional support for spin-triplet pairing in $\mathrm{UNi}_{2} \mathrm{Al}_{3}$ [280].

Indirect probes of the superconducting energy gap symmetry for $\mathrm{UPd}_{2} \mathrm{Al}_{3}$ and $\mathrm{UNi}_{2} \mathrm{Al}_{3}$ have obtained evidence for the presence of nodes where $\Delta(\vec{k})=0$. The observed temperature dependence of the specific heat in the superconducting state of $\mathrm{UPd}_{2} \mathrm{Al}_{3}$ goes as $C=\gamma T+A T^{3}$ [237], indicating a residual contribution to $C / T$ at $0 \mathrm{~K}$ from ungapped states. This finding is consistent with the presence of point nodes in $\Delta(\vec{k})$, inferred from $C \propto T^{3}$ behavior [60]). On the other hand, angle-resolved magnetothermal transport measurements support the presence of a line node in $\Delta(\vec{k})$ for $\mathrm{UPd}_{2} \mathrm{Al}_{3}$ [239]. NMR measurements provide additional support for an anisotropic energy gap containing a line node [240].

Tunneling measurements are extremely challenging to make on heavy-fermion superconductors due to their short superconducting coherence lengths, which require extremely clean sample surfaces to resolve a signal; however, tunneling spectroscopy measurements were successfully performed on epitaxial thin films of $\mathrm{UPd}_{2} \mathrm{Al}_{3}$ [281]. A strong coupling feature between the charge carriers and an antiferromagnetic spin fluctuation was observed in the tunneling conductivity of $\mathrm{UPd}_{2} \mathrm{Al}_{3}$
[281]; when this result is considered together with results from neutron-spectroscopy measurements, a compelling argument for spin-fluctuation mediated pairing in $\mathrm{UPd}_{2} \mathrm{Al}_{3}$ can be made [281].

\subsection{2. $\mathrm{UPt}_{3}$}

The compound $\mathrm{UPt}_{3}$ is isostructural with the nonsuperconducting heavy-fermion compound $\mathrm{CeAl}_{3}$ [9], forming with the hexagonal space group $P 6_{3} / m m c$. While searching for evidence of strong spin fluctuations, bulk superconductivity was observed in $\mathrm{UPt}_{3}$ in 1984 at $T_{c}=0.54 \mathrm{~K}$ [53]. With a measured $\gamma$ value of $420-450 \mathrm{~mJ} \mathrm{~mol}^{-1} \mathrm{~K}^{-2}$ in the normal state [282, 53, 233], $\mathrm{UPt}_{3}$ was quickly compared to the other known heavy-fermion superconductors at the time $\left(\mathrm{UBe}_{13}\right.$ and $\mathrm{CeCu}_{2} \mathrm{Si}_{2}$ ). Effective quasiparticle masses of $m^{*} / m_{0}=187$ and 240 were estimated from analysis of the upper critical fields [283] and far infrared absorptivity [284], respectively, while analysis of quantum oscillation studies obtained effective masses as high as $m^{*} / m_{0}=120[285,286]$. These enhanced effective quasiparticle masses firmly classify $\mathrm{UPt}_{3}$ as a heavyfermion superconductor.

In its normal state, the magnetic susceptibility of $\mathrm{UPt}_{3}$ is anisotropic, exhibiting Curie-Weiss behavior at high temperatures [282] that is also observed in NMR measurements [287]. Studies conducted using numerous probes were initially unable to detect any evidence for magnetic order in $\mathrm{UPt}_{3}$; however, measurements of $\mu \mathrm{SR}$ [231] and neutron scattering were eventually able to resolve evidence for commensurate antiferromagnetic order with a small ordered magnetic moment of $0.02 \mu_{B} / \mathrm{U}$ [232]. The apparent absence of a feature near $T_{N}=5 \mathrm{~K}$ in ${ }^{195} \mathrm{Pt}$ NMR [287] and specific heat [53, 233] measurements has led some to suggest that magnetic order in $\mathrm{UPt}_{3}$ is relatively dynamic.

Evidence for multiple intrinsic superconducting phases in $\mathrm{UPt}_{3}$, separated by 50-60 mK, was observed in high-resolution specific heat measurements [233, 288]. Considering the results from numerous studies, Joynt and Taillefer state that the two transitions in $\mathrm{UPt}_{3}$ occur at $T_{c}^{+}=0.53 \mathrm{~K}$ with specific heat jump $\Delta C / \gamma T_{c}^{+} \simeq 0.55$ and $T_{c}^{-}=0.48 \mathrm{~K}$ with jump $\Delta C / \gamma T_{c}^{-} \simeq 0.27$ [289]. Fisher et al. noted that they could obtain roughly universal values $\Delta C / \gamma T_{c} \simeq 1$ if they normalized the specific-heat jump by the fraction of electrons contributing to the superconducting energy gap $f_{s}=\left(\gamma-\gamma_{0}\right) / \gamma$ where $\gamma_{0} \sim 56-265 \mathrm{~mJ} \mathrm{~mol}^{-1}$ $\mathrm{K}^{-1}$ (depending on the sample) is a residual electronic contribution to specific heat at $0 \mathrm{~K}$ [233]. Studies of the specific heat under applied magnetic field demonstrated that there are actually three distinct superconducting phases in $\mathrm{UPt}_{3}$, denoted $\mathrm{A}$, B, and C (see the $H-T$ phase diagram in Fig. 9) [288].

Early studies on $\mathrm{UPt}_{3}$, prior to the discovery of distinct superconducting phases, observed significant anisotropy in the upper critical fields $H_{c 2}(T)$ [283]. In experiments performed as low as $150 \mathrm{mK}$, the measured $H_{c 2}(0)$ values exceeded the Pauli paramagnetic limiting field, estimated to be $H_{p 0} \simeq 0.957 \mathrm{~T}$, by a factor of nearly 2 and the presence of spin-orbit scattering (not accounted for in the formula $H_{p 0} \simeq 1.84 T_{c}$ [71]) was invoked as the explanation [283]. However, using the measured value of $\left(d H_{c 2} / d T\right)_{T_{c}} \simeq-6.3 \mathrm{~T} / \mathrm{K}$ and $T_{c}$, an orbital upper critical 


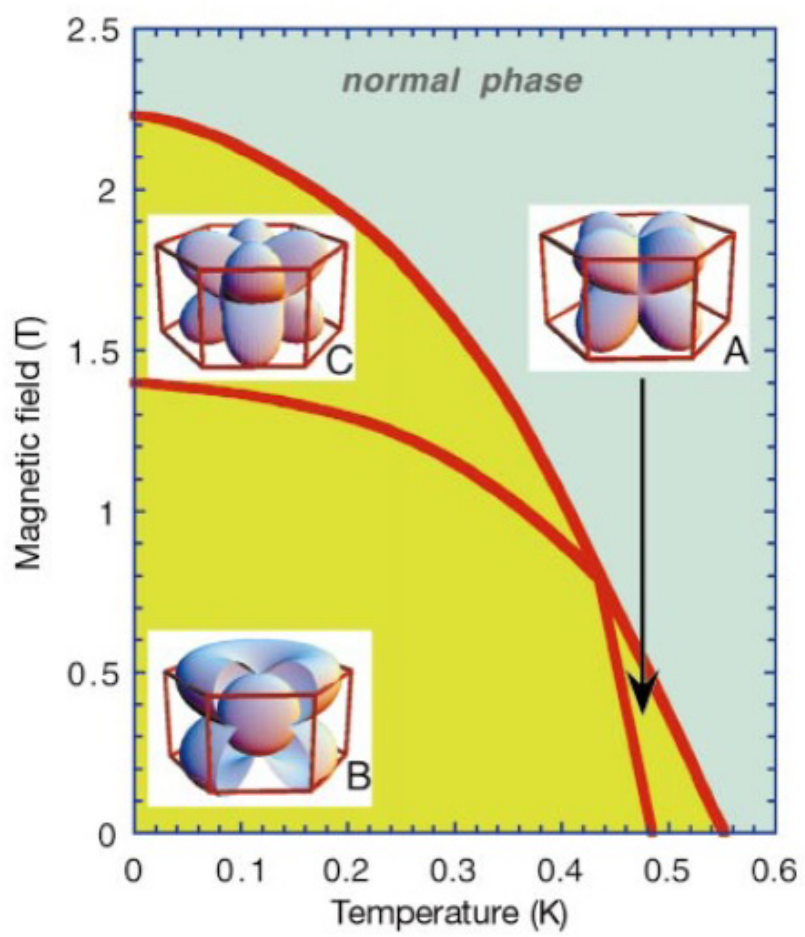

Figure 9: (Color online) The magnetic field-temperature $(H-T)$ phase diagram for $\mathrm{UPt}_{3}$ for $H \| c$ is displayed. Three distinct superconducting phases, denoted A, B, and C, are depicted. The theoretical superconducting energy gap symmetry for each phase is shown schematically for the $E_{2 u}$ theoretical model, and the surrounding frames define the hexagonal crystal symmetry of $\mathrm{UPt}_{3}$. The presence of nodes in the gaps is evident, and is most easily seen in the cut-away schematic of the gap for phase B where the gap intersects the internal sphere representing the Fermi surface (after Ref. [234]).

field of $H_{c 2}^{*}(0)=2.27 \mathrm{~T}$ is estimated [283], which is not dissimilar to $H_{c 2}(0)$ values. Measurements of $H_{c 2}(T)$ for $\mathrm{UPt}_{3}$ to lower temperatures demonstrated that the anisotropy, characterized by $H_{c 2}^{\|}(T)>H_{c 2}^{\perp}(T)$ near $T_{c}$ (where $\|$ and $\perp$ are relative to the crystallographic $c$ axis), reverses below $\sim 0.2 \mathrm{~K}$ so that $H_{c 2}^{\|}(0)<H_{c 2}^{\perp}(0)$ [290]. With the discovery of multiple superconducting phases in $\mathrm{UPt}_{3}[233,288]$, it was realized that each phase has its own characteristic upper critical fields. Therefore, the measured zero-temperature upper critical fields $H_{c 2}(0)$ are associated with the $\mathrm{C}$ phase, while $H_{c 2}\left(T \simeq T_{c}^{+}\right)$values are characteristic of the A phase [289]. The observed anisotropy of $H_{c 2}(T)$ in $\mathrm{UPt}_{3}$ is related to anisotropic mass enhancements of the quasiparticles as confirmed in quantum oscillation studies [286].

Measurements of the specific heat of $\mathrm{UPt}_{3}$ under applied pressure demonstrated that the zero-magnetic field superconducting phases (A and $\mathrm{B}$ ) are suppressed at different rates $\left(d T_{c}^{+} / d P \simeq-0.24(5) \mathrm{K} / \mathrm{GPa}\right.$ and $\left.d T_{c}^{-} / d P \simeq-0.05(1) \mathrm{K} / \mathrm{GPa}\right)$ with the two transitions merging at approximately $0.37 \mathrm{GPa}$ and $419 \mathrm{mK}$ [266]. The ordered magnetic moment from $\mathrm{U}$ ions is suppressed by applying pressure, as determined in neutron scattering experiments, vanishing near 0.3-0.4 GPa at 1.8 K [291]. This apparent correlation between the suppression of antiferromagnetic order and the emergence of a single superconducting state strongly suggests that the subtle and dynamic antiferromagnetic order in $\mathrm{UPt}_{3}$ plays a role in the emergence of the distinct superconducting states.

The coexistence of spin fluctuations and superconductivity and the upper critical field results, namely $H_{c 2}(0)>H_{p 0}$, both motivated early discussions of a possible $p$-wave orderparameter symmetry for $\mathrm{UPt}_{3}[53,283]$. It has also been claimed that the strong sensitivity of the superconducting properties to annealing and other sample synthesis conditions is consistent with this possibility [53,292]. Nodes in the superconducting energy gap are suggested by the observation of a contribution to the specific heat in the superconducting state with linear temperature dependence (i.e., $C / T$ is non-zero as $T \rightarrow$ $0 \mathrm{~K}$ ) and relatively small $\Delta C / \gamma T_{c}$ jumps in the specific heat at $T_{c}^{+}$and $T_{c}^{-}[233,292]$; both observations are consistent with ungapped electron states. The distinct nodal structures of the three superconducting phases of $\mathrm{UPt}_{3}$, proposed based on a study of the flux lattice using neutron scattering measurements, are depicted in Fig. 9 from Ref. [234]. The superconducting order parameter of $\mathrm{UPt}_{3}$ has been discussed in detail in Refs. [293, 289], but it remains a subject of continued experimental interest and attention. Recently, a complex order parameter for $\mathrm{UPt}_{3}$ was observed through angle-resolved measurements of the critical currents in Josephson tunnel-junction experiments; the results were consistent with an $E_{2 u}$ order-parameter symmetry with an odd-parity triplet representation [294]. More recently, the polar Kerr effect was observed only in the B phase of $\mathrm{UPt}_{3}\left(T<T_{c}^{-}\right.$ and $H=0 \mathrm{~T}$ ), providing evidence for a complex-two component order parameter and time-reversal symmetry breaking within the B phase [295].

\subsection{3. $U R u_{2} S i_{2}$}

The compound $\mathrm{URu}_{2} \mathrm{Si}_{2}$ is one of the most intriguing and enigmatic heavy-fermion superconductors known. This compound has the tetragonal $\mathrm{ThCr}_{2} \mathrm{Si}_{2}$ structure, which is shared by many Ce-based heavy-fermion superconductors and a class of Fe pnictide and chalcogenide high-temperature superconductors with the formula $\mathrm{MFe}_{2} \mathrm{X}_{2}$, where $\mathrm{M}$ is an alkali metal, alkaline earth, or lanthanide, and $\mathrm{X}$ is a pnictogen or chalcogen.

At ambient pressure, $\mathrm{URu}_{2} \mathrm{Si}_{2}$ exhibits two ordered phases: a so-called "hidden order" (HO) phase below $T_{o}=17.5 \mathrm{~K}$ and a superconducting phase below $T_{c}=1.5 \mathrm{~K}$, which coexist with one another $[54,55,56]$. The terminology "hidden order" refers to the fact that the identity of the order parameter (OP) of the HO phase has eluded researchers for nearly three decades. Based on an analysis of the mean-field like anomaly in the specific heat of $\mathrm{URu}_{2} \mathrm{Si}_{2}$, two of the authors and their coworkers [55] proposed a partial gapping scenario in which the HO phase, conjectured to be a charge or spin density wave (CDW or SDW), forms a gap of $\sim 130 \mathrm{~K}$ over $\sim 40 \%$ of the Fermi surface, while the remainder of the Fermi surface is gapped by the superconductivity that occurs at lower temperature. Energy gaps with comparable values have been extracted from various physical properties including, for example, electrical resistivity [296], optical conductivity spectra [297], and scanning tunneling microscopy [298, 299]. Neutron scattering experiments on $\mathrm{URu}_{2} \mathrm{Si}_{2}$ single crystal specimens revealed that 
the $\mathrm{U}$ ions exhibit antiferromagnetic order in the HO phase in which the $\mathrm{U}$ magnetic moments are aligned along the $c$-axis direction and have magnitudes of only $0.03 \mu_{B} / \mathrm{U}[300,301]$. However, the reduction in entropy associated with the HO transition is $\sim 0.2 R \ln 2$ [55] is much larger than that which would be expected for the ordering of such small $U$ magnetic moments (hence the terminology "hidden order"). An extensive review of the hidden order, superconductivity, magnetism and other aspects of $\mathrm{URu}_{2} \mathrm{Si}_{2}$ can be found in a recent review article by Mydosh and Oppeneer [302].

The delicate interplay of competing interactions in $\mathrm{URu}_{2} \mathrm{Si}_{2}$ can be "tuned" by varying control parameters such as pressure, magnetic field, and composition of elemental substituents, yielding a variety of correlated electron ground states. Upon the application of pressure, $\mathrm{URu}_{2} \mathrm{Si}_{2}$ undergoes a first order transition [303] from the HO phase to a large moment antiferromagnetic (LMAFM) phase at a critical pressure $P_{c}$, reported by various groups to lie in the range $(0.5-1.5 \mathrm{GPa})$ [304]. The magnetic structure of the LMAFM phase, in which the magnetic moment is $\sim 0.4 \mu_{B} / \mathrm{U}$, is identical to the magnetic structure of the $\mathrm{HO}$ phase $[305,302]$. It is widely believed that the small moment antiferromagnetic (SMAFM) order in the HO phase is extrinsic and associated with a small volume fraction of the LMAFM phase that is produced by local strains that increase the $c / a$ ratio over a critical value [306]. However, some researchers believe that the SMAFM order in the HO phase is intrinsic, since the onset of the SMAFM occurs at $T_{o}$ and it is observed in samples with different residual resistivity values [307].

The application of a large magnetic field $H$ results in a suppression of the $\mathrm{HO}$ phase at $H \approx 35 \mathrm{~T}$ and the emergence of several novel quantum phases that exhibit non-Fermi liquid behavior [308]. The substitution of transition elements for $\mathrm{Ru}$ from neighboring columns in the periodic table have been found to suppress the $\mathrm{HO}$ and to generate other magnetic phases [309, 310, 311, 312], while substitution of elements from the same column of the periodic table have been found to enhance the $\mathrm{HO}[311,313,314]$. The substitution of Rh generates LMAFM phases [312], while the substitution of $\mathrm{Mn}, \mathrm{Tc}$ and Re induces an itinerant heavy electron ferromagnetic phase [309, 310]. Non-Fermi liquid characteristics in the physical properties that persist deep into the ferromagnetic state in the $\mathrm{URu}_{2-x} \mathrm{Re}_{x} \mathrm{Si}_{2}$ system have been reported $[315,316]$. The substitution of $\mathrm{Fe}$ and $\mathrm{Os}$ for $\mathrm{Ru}$ produces a large twofold enhancement of the HO/LMAFM phase boundary with a "kink" at a critical substituent concentration that is apparently associated with a transition from the HO to the LMAFM phase [313, 314]. It has been suggested that the enhancement of the HO/LMAFM phase boundary for Fe substitution is driven by "chemical pressure" generated by substitution of smaller Fe ions for Ru ions [313]; additional evidence supporting this interpretation was presented in a recent study of specific heat and neutron diffraction measurements on $\mathrm{URu}_{2-x} \mathrm{Fe}_{x} \mathrm{Si}_{2}$ single crystals [317]. However, this argument cannot explain the enhancement of the HO/LMAFM phase boundary upon substitution of larger Os ions for $\mathrm{Ru}$ ions [311, 314].

$\mathrm{URu}_{2} \mathrm{Si}_{2}$ exhibits a type of unconventional $d$-wave spin-

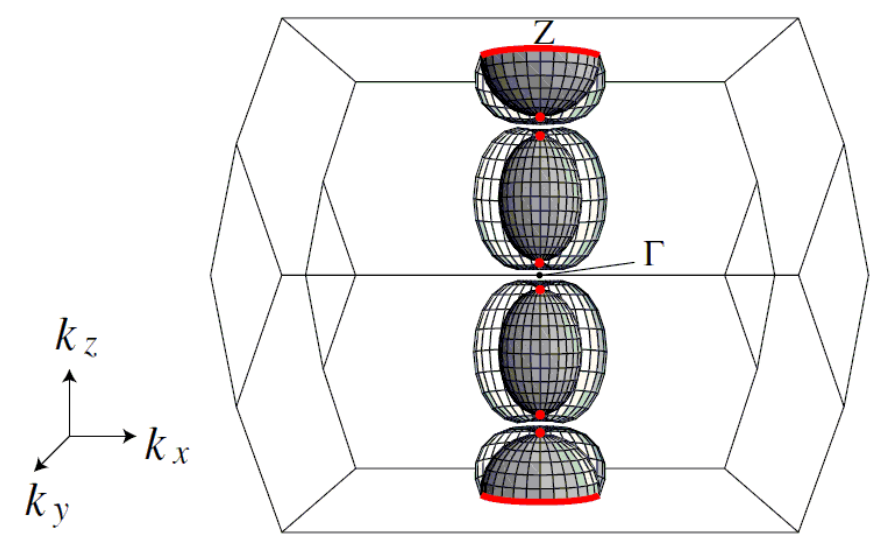

Figure 10: (Color online) Schematic of the Fermi surface (opaque) and superconducting energy gap structure (transparent) of $\mathrm{URu}_{2} \mathrm{Si}_{2}$ after Ref. [328].

singlet multiband superconductivity with two distinct superconducting energy gaps. The jump $\Delta C$ in the specific heat at $T_{c}$ decreases rapidly with pressure and is no longer detectable at pressures within the HO phase $[318,319,320]$. Insofar as this can be interpreted as a measure of bulk superconductivity, it indicates that bulk superconductivity only occurs within the HO phase and not in the LMAFM phase. In contrast, the resistive superconducting transitions extend well into the LMAFM phase, indicating that either filamentary superconductivity or superconducting fluctuations extend into the LMAFM phase [321].

Measurements of the specific heat in the superconducting state at low temperatures reveal a $T^{2}$ dependence that is consistent with line nodes in the energy gap [318, 292]. The ${ }^{29} \mathrm{Si}$ NMR relaxation rate, $T_{1}$, varies as $T^{3}$ in the superconducting state at low temperature, which is also consistent with line nodes [322, 323]. Point-contact spectroscopy measurements on a $\mathrm{URu}_{2} \mathrm{Si}_{2}$ single crystal yield a superconducting energy gap that is not consistent with BCS behavior and evidence for a pseudogap between $T_{c}=1.37 \mathrm{~K}$ and $2 \mathrm{~K}$ [324]. Quantum oscillation [325, 326, 327], electronic transport [328], specific heat [329], and thermal conductivity [328] measurements indicate that $\mathrm{URu}_{2} \mathrm{Si}_{2}$ is a multiband superconductor containing a light spherical hole band and an anisotropic heavy electron band. Angle-resolved thermal conductivity and specific heat measurements have suggested two distinct superconducting gap structures having different nodal topology with horizontal line nodes in the hole band and point nodes in the electron band (see Fig. 10) [328, 329, 330, 331]. From a symmetry group analysis, a chiral $d$-wave state that breaks time-reversal symmetry has been proposed [328]. Evidence for time-reversalsymmetry-breaking has recently been reported by Schemm et al. [332]. There have been several studies of flux line lattice melting phenomena in $\mathrm{URu}_{2} \mathrm{Si}_{2}$ polycrystals [333] and single crystals [334].

\subsection{Interplay of Ferromagnetic Order and Superconductivity}

There are four U-based heavy-fermion compounds in which the interplay between ferromagnetism and superconductivity 
plays a significant role: $\mathrm{UGe}_{2}$ [245], URhGe [247], UCoGe [249], and UIr [254, 255]. The properties of these four heavyfermion superconductors are reviewed in this special issue of Physica $\mathrm{C}$ in the article on ferromagnetic superconductors by Andrew Huxley. Furthermore, the compound UIr crystallizes with a noncentrosymmetric crystal structure, so its properties are also covered in this special issue's article on noncentrosymmetric superconductors by F. Kneidinger et al.. However, in addition to providing some pertinent information concerning the superconducting and normal-state properties of these compounds in Table 3, we would like to touch briefly on the compounds $\mathrm{UGe}_{2}$ and $\mathrm{UCoGe}$ below.

\subsection{1. $U G e_{2}$}

Ferromagnetic order with an ordered magnetic moment of $1.48 \mu_{B} / \mathrm{U}$ develops parallel to the short orthorhombic $a$ axis in $\mathrm{UGe}_{2}$ below $T_{C}=52 \mathrm{~K}$ [243]. The effective magnetic moment, obtained from Curie-Weiss analysis of magnetic susceptibility data, was determined to be $2.7 \mu_{B} / \mathrm{U}$ [243]; this value is smaller than the saturation magnetic moment, which is consistent with itinerant $\mathrm{U} 5 f$ electron states in $\mathrm{UGe}_{2}$ [49]. Analysis of specific heat data at low temperature reveals that $\gamma \simeq 32 \mathrm{~mJ}$ mol $^{-1} \mathrm{~K}^{-2}$, suggesting modestly-enhanced quasiparticle masses in $\mathrm{UGe}_{2}$ [244]. A subtle feature is observed in $\rho(T)$ data at $T_{x} \approx$ $25 \mathrm{~K}$ [335], below which, the ferromagnetic magnetic moment increases, suggesting that there are actually two distinct ferromagnetic phases in $\mathrm{UGe}_{2}$ [336]. Under applied hydrostatic pressure, both $T_{C}$ and $T_{x}$ are suppressed monotonically. The ferromagnetic phase transition below $T_{C}$ becomes first order above $\sim 1.2 \mathrm{GPa}[244]$ and $T_{C}$ extrapolates to $0 \mathrm{~K}$ near $1.6 \mathrm{GPa}$ [245, 244, 337]. The abrupt, first-order decrease of $T_{C}(P)$ at the critical pressure is especially dramatic in measurements on polycrystalline samples [338]. The other temperature scale, $T_{x}$, is expected to vanish near $1.2 \mathrm{GPa}$; however, the suppression is veiled by the emergence of a dome of superconductivity extending from near $1 \mathrm{GPa}$ to $1.6 \mathrm{GPa}$ with maximum $T_{c}=0.8 \mathrm{~K}$ at $\sim 1.2 \mathrm{GPa}[245,244,337]$. Near $1.2 \mathrm{GPa}, \gamma \simeq 110 \mathrm{~mJ} \mathrm{~mol}^{-1}$ $\mathrm{K}^{-2}$ [246], indicating a strong enhancement of the quasiparticle masses relative to the ambient-pressure result. An enhancement of $\gamma(P)$ was observed in the pressure range where superconductivity emerges in polycrystalline samples [339]. The jump at $T_{c}$ in specific heat data, $\Delta C / \gamma T_{c} \simeq 0.2-0.3$, observed under an applied pressure of $1.13 \mathrm{GPa}$, is small but consistent with bulk superconductivity [246].

The superconducting state of $\mathrm{UGe}_{2}$ resides completely within the ferromagnetic phase, with each vanishing under an applied pressure of $1.6 \mathrm{GPa}[245,244,337]$. This observation strongly suggests that ferromagnetic order and superconductivity do not merely coexist, but that the mechanism underlying the emergence of superconductivity in $\mathrm{UGe}_{2}$ depends upon the presence of ferromagnetic order. Attempts to elucidate the nature of the electron pairing in $\mathrm{UGe}_{2}$ have led to odd-parity equal-spin triplet pairing as the most promising candidate [49]. However, studies on polycrystalline samples of $\mathrm{UGe}_{2}$ with $\rho_{0}$ values at least an order of magnitude higher than those in single crystalline samples were able to resolve a pressure-temperature phase diagram [338] that is similar to that in Refs. [245, 244, 337] (constructed from measurements on single crystals) and includes robust, bulk superconductivity as evidenced by jumps in specific heat at $T_{c}$ [339]. These results may be inconsistent with a $p$-wave pairing symmetry for superconductivity in $\mathrm{UGe}_{2}$, which should be extremely sensitive to disorder, especially when the mean free path approaches the coherence length. The small jump $\Delta C / \gamma T_{c}$ in specific heat at $T_{c}$ and a residual value of $C / T$ as $T \rightarrow 0 \mathrm{~K}$ suggest that there are nodes in the superconducting energy gap of $\mathrm{UGe}_{2}$ [246]; more specifically, line nodes are suggested [60] by the linear temperature dependence of $C / T$ in the superconducting state.

\subsection{2. $U C o G e$}

Unlike $\mathrm{UGe}_{2}$ [245] and URhGe [247], for which superconductivity resides deep within ferromagnetically-ordered states with large ordered moments, superconductivity in UCoGe coexists with a weak itinerant ferromagnetic order. In polycrystalline samples of UCoGe and at ambient pressure, ferromagnetic order is observed below $T_{C}=3 \mathrm{~K}$ with an ordered magnetic moment of $0.03 \mu_{B} / \mathrm{U}$ (polarized along the $c$ axis [250]) with superconductivity being observed near $T_{c}=0.8 \mathrm{~K}$ [249]. An effective magnetic moment from Curie-Weiss behavior analysis of $1.7 \mu_{B} / \mathrm{U}$ is much larger than the ordered moment and the magnetic entropy released at $T_{C}\left(S_{\text {mag }} \approx 0.03 R \ln 2\right)$ is small; both results are consistent with weak itinerant ferromagnetic order [249]. $\mu \mathrm{SR}$ measurements demonstrate that this weak ferromagnetism coexists with superconductivity on a microscopic scale [340]. In measurements of specific heat, a Sommerfeld coefficient $\gamma=57 \mathrm{~mJ} \mathrm{~mol}^{-1} \mathrm{~K}^{-2}$ is obtained, suggesting that the effective quasiparticle masses are moderatelyenhanced, and a jump at $T_{c}, \Delta C / \gamma T_{c} \simeq 1$, indicates that superconductivity in UCoGe is bulk [249]. The physical properties of UCoGe are strongly sample-dependent [249] and high-quality single crystals tend to have transition temperatures $T_{C}=2.5 \mathrm{~K}$ and $T_{c}=0.5-0.6 \mathrm{~K}[251,253,252]$.

Significant anisotropy was observed in the upper critical field curves measured on single-crystalline samples of UCoGe [250]. The results include: large value of $H_{c 2}(0) \simeq 5 \mathrm{~T}$ for $H \| a, b$; large anisotropy, $H_{c 2}^{a}(0) \simeq H_{c 2}^{b}(0)>>H_{c 2}^{c}(0)$, of a factor $\sim 10$; a pronounced upturn of $H_{c 2}(T)$ with decreasing temperature along all three principle axes [250]. The upper critical fields $H_{c 2}(T)$ along $H \| a, b$ significantly exceed the Pauli paramagnetic limit. The anisotropy and values of $H_{c 2}(0)$ provide evidence for spin-triplet pairing and a superconducting gap with axial symmetry and point nodes along the $c$ axis [250]. Neutron scattering measurements also suggest nodes are present in the superconducting energy gap along the $c$ axis [341]. Angleresolved NMR measurements on UCoGe demonstrate that magnetic fields applied along the $c$-axis strongly suppress critical ferromagnetic spin fluctuations [342, 253], and this suppression is believed to be intimately coupled to the unusual anisotropic behavior of $H_{c 2}(T)$ in UCoGe [253]. Furthermore, it has been suggested that the critical ferromagnetic spin fluctuations with Ising anisotropy may mediate spin-triplet superconductivity in UCoGe [253].

Under applied pressure, $T_{C}$ is suppressed at a rate of $1.4 \mathrm{~K} / \mathrm{GPa}$, while $T_{c}$ is relatively unaffected up to $2.4 \mathrm{GPa}$ 
as it traces out a superconducting dome with weak curvature [343, 251]. In studies on a single crystal, ferromagnetic order is completely suppressed near $1.4 \mathrm{GPa}$, and unlike in the other ferromagnetic superconductors, superconductivity in UCoGe persists into the paramagnetic state at pressures above $1.4 \mathrm{GPa}$ [251]. From symmetry considerations, it is expected that the superconductivity in the paramagnetic state is distinct from the superconducting state that coexists with weak itinerant ferromagnetism below 1.4 GPa [251]. The spectacular enhancement of the $H_{c 2}(T)$ curves under applied pressure is certainly consistent with such a scenario [251]. Spin-triplet pairing and the presence of a ferromagnetic quantum critical point near $1.4 \mathrm{GPa}$ are strongly implied by these results. It may also be possible to access such a quantum critical point via chemical substitution as has been reported near $x=0.22$ in the alloy system $\mathrm{UCo}_{1-x} \mathrm{Fe}_{x} \mathrm{Ge}[344]$.

\section{4. $U_{6} T, U_{2} P t C_{2}$, and $U B e_{13}$}

As we did in Section 2, we conclude our discussion of Ubased heavy-fermion superconductors with three examples that do not fall neatly into the categories of residing near or coexisting with antiferromagnetic or ferromagnetic order. The first is a family of isostructural compounds $\mathrm{U}_{6} \mathrm{~T}(\mathrm{~T}=\mathrm{Mn}, \mathrm{Fe}, \mathrm{Co}$, $\mathrm{Ni}$ ), the second is the compound $\mathrm{U}_{2} \mathrm{PtC}_{2}$, and the third is the compound $\mathrm{UBe}_{13}$.

The compounds $\mathrm{U}_{6} \mathrm{~T}(\mathrm{~T}=\mathrm{Mn}, \mathrm{Fe}, \mathrm{Co}, \mathrm{Ni})$ are among the earliest superconducting compounds comprised of magnetic $3 d$ transition metals to be discovered [265]. Hill and Matthias [345] observed that the dependence of the $T_{c}$ of pseudobinary $\mathrm{U}_{6} \mathrm{~T}_{1-x} \mathrm{~T}_{x}^{\prime}$ alloys on the $d$-element valence electron count is strikingly similar to the "Slater-Pauling" curve that describes the variation of the saturation magnetic moment with electron density for $\mathrm{T}_{1-x} \mathrm{~T}_{x}^{\prime}$ transition-metal alloys. This led them to speculate that a "magnetic mechanism" was responsible for the superconductivity of the $\mathrm{U}_{6} \mathrm{~T}$ compounds [345]. This intriguing suggestion foreshadowed the current interpretation concerning the pairing mechanism of many unconventional superconductors. Subsequent measurements of the specific heat, magnetic susceptibility, and the upper critical magnetic field [225, 224], revealed that $\mathrm{U}_{6} \mathrm{Fe}$ and $\mathrm{U}_{6} \mathrm{Co}$ are moderately heavy Fermiliquid systems with electronic effective masses $m^{*} / m_{0} \simeq 20$. These properties have led many to compare $\mathrm{U}_{6} \mathrm{Fe}$ with $\mathrm{UPt}_{3}$ and $\mathrm{UBe}_{13}$ and count it among the heavy-fermion superconductors [346]; however, this interpretation is not universally accepted, and many consider $\mathrm{U}_{6} \mathrm{Fe}\left(T_{c} \simeq 3.8 \mathrm{~K}\right)$ and the $\mathrm{Pu}$ - and Np-based superconductors (see Section 4.3) to constitute links between heavy-fermion superconductors and other unconventional superconductors such as the cuprates.

Superconductivity was reported in the compound $\mathrm{U}_{2} \mathrm{PtC}_{2}$ with $T_{c}=1.47 \mathrm{~K}$ by Matthias et al. [227]. Subsequent studies demonstrated that there is no magnetic order in $\mathrm{U}_{2} \mathrm{PtC}_{2}$ [228, 229]; though, evidence for strong ferromagnetic fluctuations has been obtained in the superconducting state from measurements of a modified Korringa law in the spin-lattice relaxation rate $1 / T_{1}$ [229]. The properties of $\mathrm{U}_{2} \mathrm{PtC}_{2}$ have been used to place it in an intermediate position between $\mathrm{U}_{6} \mathrm{Fe}$ and the unambiguous heavy-fermion compounds $\mathrm{UPt}_{3}$ and $\mathrm{UBe}_{13}$ [228].

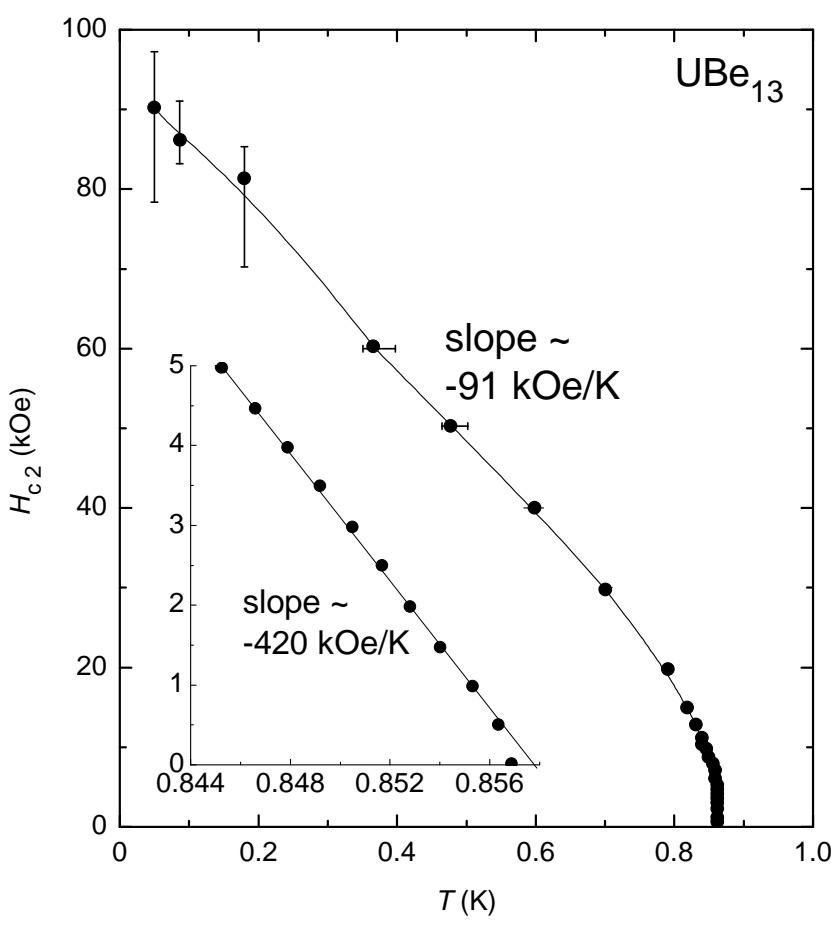

Figure 11: Upper critical field curve for $\mathrm{UBe}_{13}, H_{c 2}(T)$, after Ref. [347].

Specific heat measurements confirm bulk superconductivity by observing a jump at $T_{c}[228,348]$ and provide a Sommerfeld coefficient of $\gamma=75 \mathrm{~mJ}$ mol- $\mathrm{U}^{-1} \mathrm{~K}^{-2}$ [228]. This value of $\gamma$, which is consistent with moderately-enhanced quasiparticle mass, has been used to demonstrate a possible correlation between $\gamma$ and the U-U separation for U-based superconducting compounds [228]. With increasing pressure, the electrical resistivity in the normal state just above $T_{c}$ decreases, and $T_{c}$ is suppressed linearly at a rate of $d T_{c} / d P \simeq-0.53 \mathrm{~K} / \mathrm{GPa}$ [349]. Early measurements of the upper critical fields on polycrystalline samples found a large initial slope of $\left(d H_{c 2} / d T\right)_{T_{c}} \simeq-9$ $\mathrm{T} / \mathrm{K}$ [228]. More recent measurements of $H_{c 2}(T)$ indicate that $H_{c 2}^{c}(0) \simeq 7.8 \mathrm{~T}$ and $H_{c 2}^{a b}(0) \simeq 9.2 \mathrm{~T}$ [229]; these values significantly exceed the estimated Pauli limiting field of $H_{p 0} \simeq 2.7 \mathrm{~T}$, but are comparable to the orbital upper critical field $H_{c 2}^{*}(0) \simeq$ 10.2 T [229], estimated using the WHH theory [59].

When $H_{c 2}(0)>>H_{p 0}$ as it is in $\mathrm{U}_{2} \mathrm{PtC}_{2}$ [229], the possibility of spin-triplet pairing can be considered. NMR measurements of the ${ }^{195} \mathrm{Pt}$ nucleus in $\mathrm{U}_{2} \mathrm{PtC}_{2}$ samples observe a temperatureindependent Knight shift, which is consistent with spin-triplet pairing [229]. Furthermore, $1 / T_{1}$ data follow a $T^{2}$ temperature dependence in the superconducting state, which is consistent with nodes in the energy gap. On the other hand, strong spin orbit scattering can also increase the paramagnetic limiting field and reduce the Knight shift of a spin-singlet superconductor. Moreover, a recent study of the system $\mathrm{U}_{2} \mathrm{Rh}_{1-x} \mathrm{Pt}_{x} \mathrm{C}_{2}$ (the compound $\mathrm{U}_{2} \mathrm{RhC}_{2}$ is an itinerant antiferromagnet with $T_{N} \simeq 22 \mathrm{~K}$ ) provides results that are difficult to reconcile with unconventional superconductivity in $\mathrm{U}_{2} \mathrm{PtC}_{2}$ [348]. Chemical substitution of $\mathrm{Pt}$ with $\mathrm{Rh}$ suppresses $T_{c}$, but not as rapidly as expected for a superconductor with spin-triplet pairing, which should be 
extremely sensitive to the presence of nonmagnetic impurities [348]. Superconductivity is surprisingly robust, persisting from $x=1$ to $x=0.9(10 \% \mathrm{Rh})$, where the estimated mean free path is comparable to the estimated superconducting coherence length (i.e., $l \approx \xi$ ) [348]. In such cases, superconductivity is predicted to be completely suppressed, but $T_{c}$ is only reduced from 1.45 $\mathrm{K}$ at $x=1$ to $1.09 \mathrm{~K}$ at $x=0.9$ [348]. This insensitivity of $T_{c}$ to impurities is hard to understand in the context of any non- $s$ wave type pairing [348], and suggests that additional research must be conducted on $\mathrm{U}_{2} \mathrm{PtC}_{2}$ to characterize its superconducting state.

The properties of the heavy-fermion compound $\mathrm{UBe}_{13}$ are quite extraordinary and bear some similarities with those of $\mathrm{CeCu}_{2} \mathrm{Si}_{2}$. A particularly noteworthy example is the Sommerfeld coefficient $\gamma=C(T) / T$, which increases with decreasing temperature and reaches an enormous value of $\sim 1 \mathrm{~J} \mathrm{~mol}^{-1} \mathrm{~K}^{-2}$ just above $T_{c} \simeq 0.9 \mathrm{~K}$, below which $\mathrm{UBe}_{13}$ is superconducting. The specific heat jump $\Delta C$ at $T_{c}$ is of the order of $\gamma T_{c}$, which demonstrates that the heavy quasiparticles responsible for the large value of $\gamma$ in the normal state are also involved in the superconductivity. The magnetic susceptibility has a CurieWeiss-like temperature dependence at high temperatures, but approaches a constant value $\chi(0)$ as $T \rightarrow 0 \mathrm{~K}$, indicative of a nonmagnetic ground state. Moreover, the value of $\chi(0)$ is consistent with the large value of $\gamma$; i.e., the Wilson-Sommerfeld ratio is of order unity. Magnetic penetration depth measurements on $\mathrm{UBe}_{13}$ yield a $T^{2}$ temperature dependence at low temperature, consistent with an axial state with point nodes in the energy gap [350]. In contrast, NMR measurements [351] reveal relaxation rates that are proportional to $T^{3}$ at low temperatures, indicative of a polar state with line nodes. However, NMR measurements yield $T^{3}$ behavior for nearly all other heavy-fermion superconductors, as noted by Heffner and Norman [267]. The upper critical field curve $H_{c 2}(T)$ of $\mathrm{UBe}_{13}$, shown in Fig. 11, has a very unusual shape. The value of the initial slope $\left(d H_{c 2} / d T\right)_{T_{c}} \simeq-42 \mathrm{~T} / \mathrm{K}$, is enormous and consistent with the large quasiparticle effective mass $m^{*} / m_{0} \simeq 300$ [347]. The $H_{c 2}(T)$ curve rolls over and then exhibits another linear region below $0.7 \mathrm{~K}$, with a smaller slope of $d H_{c 2} / d T \simeq$ $-9.1 \mathrm{~T} / \mathrm{K}$, that persists to very low temperatures of the order of $50 \mathrm{mK}$ [347].

Chemical substitution of $\mathrm{Th}$ for $\mathrm{U}$ in $\mathrm{UBe}_{13}$ yields a striking $T-x$ phase diagram, based on specific heat and other measurements, some of which reveal a region with multiple phase transitions; these phases consist of at least two superconducting regions, which appear to correspond to different types of superconductivity [267]. Additional evidence for the existence of two distinct types of superconductivity, presumably with different order parameter symmetries, are provided by measurements of $T_{c}$ under pressure on samples of $\mathrm{U}_{1-x} \mathrm{Th}_{x} \mathrm{Be}_{13}$ (see Fig. 12(a)) [268] and measurements of $H_{c 2}(T)$ for Gdsubstituted samples of $\mathrm{U}_{1-x} \mathrm{Th}_{x} \mathrm{Be}_{13}$ with different values of $x$ [352] in these two superconducting regions. The properties of this remarkable heavy-fermion superconductor are summarized in several reviews to which the reader is referred for further information [267, 269].

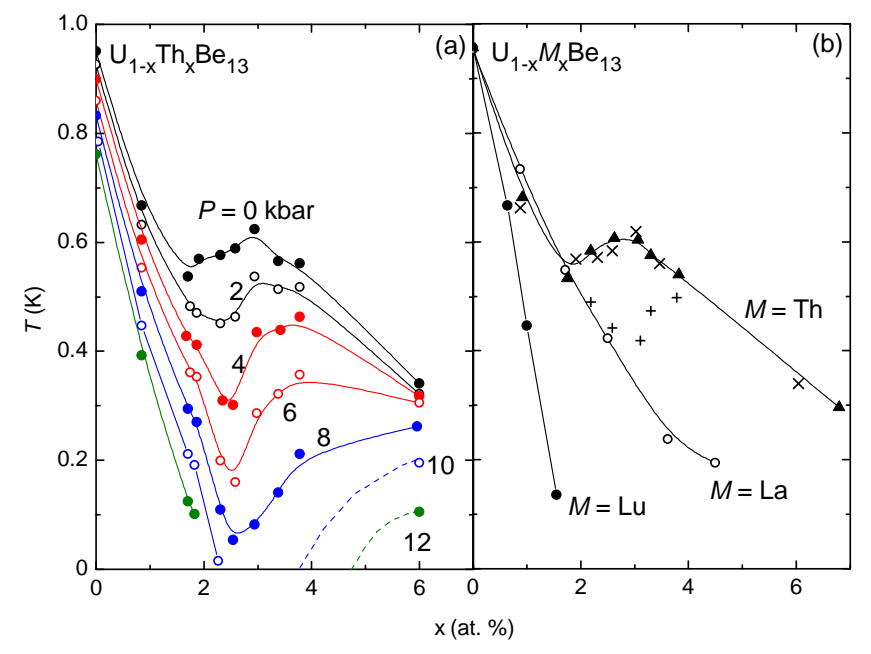

Figure 12: (Color online) (a) Superconducting critical temperature $T_{c}$ for $\mathrm{U}_{1-x} \mathrm{Th}_{x} \mathrm{Be}_{13}$ as a function of $x$ for several applied pressures (explicitly labeled in units of kbar). Lines are guides to the eye. (b) $T_{c}$ for $\mathrm{U}_{1-x} M_{x} \mathrm{Be}_{13}(M=\mathrm{La}$, $\mathrm{Lu}, \mathrm{Th})$ at ambient pressure from measurements of ac magnetic susceptibility. The data for $M=$ Th (filled triangles and crosses) are from two independent studies. Plus symbols represent a feature in measurements of specific heat for $M=$ Th samples that indicates a second phase transition within the superconducting state. Adapted from Ref. [268].

\section{Pr-, Yb-, Pu-, and Np-based heavy-fermion supercon- ductors}

\subsection{Pr-based compounds}

Compared with Ce-, U-, and Yb-based compounds, not many Pr-based heavy-fermion compounds are currently known. A brief description of the necessary materials design it required to discover the first Pr-based heavy-fermion compound, PrInAg [30], is provided in Ref. [353]. The main impediment to the formation of a heavy-fermion ground state in Pr-based compounds is that Pr is a non-Kramer's ion and, for most point symmetries, the singlet ground state that is observed does not supply the necessary degeneracy. A $\Gamma_{3}$ doublet ground state is observed in a few Pr-based heavy-fermion compounds and only occurs when Pr occupies a crystallographic position with cubic point group symmetry. Interestingly, $\Gamma_{3}$ is nonmagnetic, so the heavy-fermion states in these compounds must have a nonmagnetic origin. It is the localized electric quadrupolar moments, associated with the $\Gamma_{3}$ doublet ground state, that play a role analogous to the localized magnetic moments in conventional heavy-fermion compounds, and they can form a Kondo lattice through the two-channel or quadrupolar Kondo effect $[27,28,29]$. Therefore, it is the interplay between quadrupolar degrees of freedom and superconductivity that is typically of interest in Pr-based heavy-fermion superconductors.

\subsection{1. $\mathrm{PrOs}_{4} \mathrm{Sb}_{12}$ and $\mathrm{PrPt}_{4} \mathrm{Ge}_{12}$}

The compound $\mathrm{PrOs}_{4} \mathrm{Sb}_{12}$ is the first Pr-based heavy-fermion superconductor reported and one of the most notable filled skutterudite compounds. This compound has a large electronic specific heat coefficient $\gamma$ of $\sim 500 \mathrm{~mJ} \mathrm{~mol}^{-1} \mathrm{~K}^{-2}[32,354]$. The specific heat jump at the superconducting critical temperature 
$T_{c}$ shows an unusual double peak feature, which has been observed in numerous studies [354, 258, 355]. The ground state of $\mathrm{Pr}$ in the CEF is a singlet, which is separated from a low lying triplet first excited state by only $\sim 7 \mathrm{~K}[356,357,358]$. Various types of measurements in high magnetic fields probing the normal state properties have revealed the existence of a high-field ordered phase (see Fig. 13) [359, 360, 361, 362], which was determined by means of neutron diffraction experiments in high magnetic fields to be an antiferroquadrupolar ordered phase [363]. Since $\mathrm{PrOs}_{4} \mathrm{Sb}_{12}$ has a nonmagnetic ground state, it has been conjectured that the superconducting electron pairing may be mediated by quadrupolar fluctuations [357]. Experiments that probe the superconducting energy gap have provided evidence for both nodal and nodeless energy gaps in $\mathrm{PrOs}_{4} \mathrm{Sb}_{12}$. Transverse muon spin rotation (TF$\mu \mathrm{SR}$ ) experiments yielded a temperature dependence of the penetration depth $\lambda$ for $\mathrm{PrOs}_{4} \mathrm{Sb}_{12}$ that is consistent with an isotropic energy gap [364]. However, scanning tunneling microscopy measurements observed a gap that was open in large regions, discounting the possibility of line nodes [365], zerofield microwave penetration depth measurements revealed behavior best described with point nodes in the superconducting energy gap [256], and small-angle neutron scattering experiments reported distortions in the flux-line lattice that were attributed to gap nodes [366]. Thermal transport measurements on single crystals carried out as a function of magnetic field direction revealed two different superconducting phases. The energy gap has four or more point nodes at high fields (A phase), while it has two point nodes at the low fields (B phase) [257]. More recent studies also suggest that $\mathrm{PrOs}_{4} \mathrm{Sb}_{12}$ is a multiband superconductor [367, 368, 369, 370]. Muon-spin relaxation measurements on $\mathrm{PrOs}_{4} \mathrm{Sb}_{12}$ provide evidence for time-reversal symmetry breaking [371]. The magnetic field $H$ vs temperature $T$ phase diagram of $\mathrm{PrOs}_{4} \mathrm{Sb}_{12}$ is shown in Fig. 13.

A new class of filled skutterudites of the form $\mathrm{RPt}_{4} \mathrm{Ge}_{12}$ has recently been synthesized [372, 373], opening up an entirely new direction for filled skutterudite research. Several members of this new class exhibit superconductivity $(\mathrm{R}=\mathrm{Sr}, \mathrm{Ba}$, Th, La, Pr) where the $\mathrm{R}=$ Pr member has one of the highest values of $T_{c}$ of $\sim 7.9 \mathrm{~K}$ [373]. Recent investigations have suggested that $\mathrm{PrPt}_{4} \mathrm{Ge}_{12}$ exhibits a type of strongly-coupled unconventional superconductivity that has point nodes in the energy gap [374] and breaks time-reversal symmetry [375]. More recent studies suggest that $\operatorname{PrPt}_{4} \mathrm{Ge}_{12}$ is a multiband superconductor [376, 377, 378]. It was also recently demonstrated that introducing magnetic Ce impurities through chemical substitution for Pr either rapidly suppresses one of the energy gaps or induces a crossover from a nodal to nodeless gap symmetry [379]. It is interesting that both $\mathrm{PrPt}_{4} \mathrm{Ge}_{12}$ and $\mathrm{PrOs}_{4} \mathrm{Sb}_{12}$ exhibit similar types of multiband unconventional superconductivity, but display striking differences in electronic structure. The CEF splitting $\delta$ between the Pr ground state singlet and triplet first excited state in the two compounds differs by an order of magnitude; $\delta \approx 7 \mathrm{~K}$ for $\mathrm{PrOs}_{4} \mathrm{Sb}_{12}[356,357]$ and $\delta \approx 130 \mathrm{~K}$ for $\mathrm{PrPt}_{4} \mathrm{Ge}_{12}$ [373, 374, 380]. The electronic correlations are rather strong in $\mathrm{PrOs}_{4} \mathrm{Sb}_{12}$ as evinced by the large electronic specific heat coefficient $\gamma \sim 500 \mathrm{~mJ} \mathrm{~mol}^{-1} \mathrm{~K}^{-2}$, whereas they

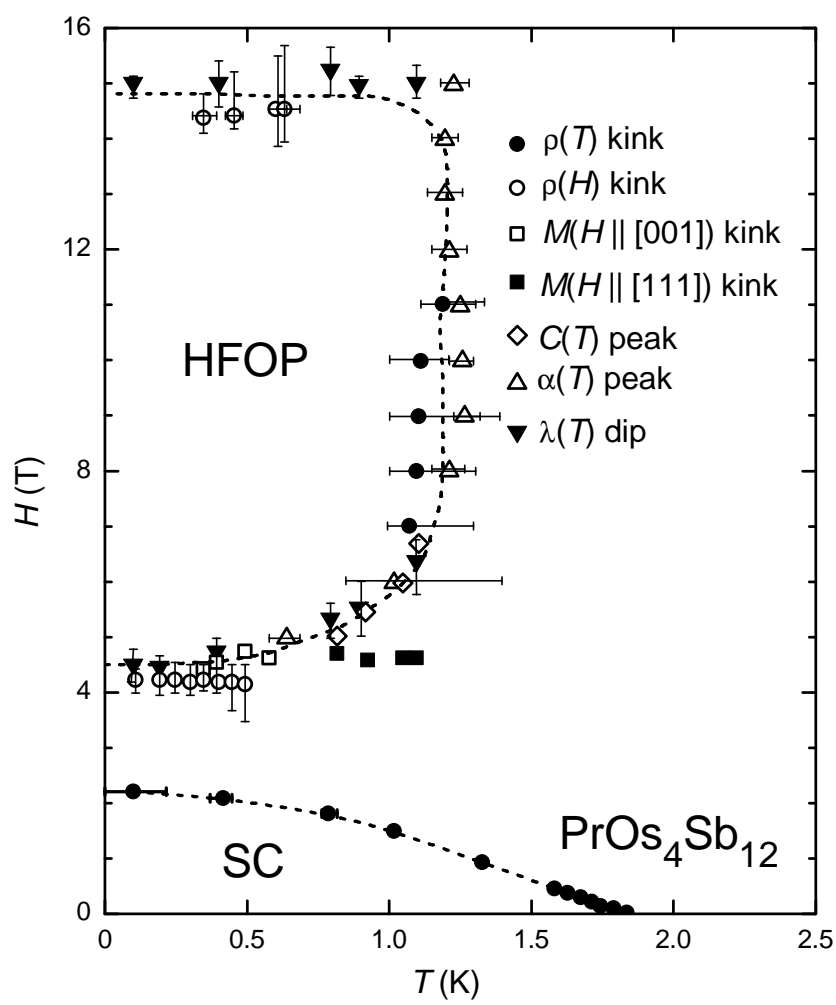

Figure 13: Magnetic field vs. temperature $(H-T)$ phase diagram for $\mathrm{PrOs}_{4} \mathrm{Sb}_{12}$ showing the high-field ordered phase, designated HFOP in the figure, which has been identified with antiferroquadrupolar order [363], and the superconducting phase (after Refs. [361, 356]).

are considerably weaker in $\mathrm{PrPt}_{4} \mathrm{Ge}_{12}$ as reflected in the much smaller value of $\gamma \sim 60 \mathrm{~mJ} \mathrm{~mol}^{-1} \mathrm{~K}^{-2}$ [373].

\subsubsection{Superconductivity in $\operatorname{Pr}_{2} X_{20}$ compounds}

Interest has been steadily growing in members of the $\mathrm{RT}_{2} \mathrm{X}_{20}$ $(\mathrm{R}=$ rare earth; $\mathrm{T}=$ transition metal; $\mathrm{X}=\mathrm{Al}, \mathrm{Zn}, \mathrm{Cd})$ family of compounds that adopt the $\mathrm{CeCr}_{2} \mathrm{Al}_{20}$-type crystal structure characterized by space group $F d \overline{3} m$. Superconductivity was first observed in 2010 among the "1-2-20" compounds, including at $T_{c} \simeq 0.05 \mathrm{~K}$ in $\operatorname{PrIr}_{2} \mathrm{Zn}_{20}$ [381]. Measurements of the specific heat of $\operatorname{PrIr}_{2} \mathrm{Zn}_{20}$ at low temperature reveal a $C / T \simeq \gamma$ value of $\sim 5 \mathrm{~J} \mathrm{~mol}^{-1} \mathrm{~K}^{-2}$ at $0.4 \mathrm{~K}[381,382]$, which was interpreted as evidence for heavy-fermion superconductivity in this compound. $\operatorname{PrIr}_{2} \mathrm{Zn}_{20}$ exhibits Curie-Weiss behavior for $T>30 \mathrm{~K}$ in magnetic susceptibility $\chi(T)$ measurements with $\mu_{e f f} \simeq 3.49(2) \mu_{B} / \operatorname{Pr}$ (associated with paramagnetic $\operatorname{Pr}^{3+}$ ions) [381, 382]; however, analysis of $\chi(T)$ data at low temperature and of a Schottky anomaly in specific heat data provided compelling evidence that is consistent with a $\Gamma_{3}$ nonmagnetic Kramers doublet ground state in $\operatorname{PrIr}_{2} \mathrm{Zn}_{20}$ [382]. Inelastic neutron scattering measurements later confirmed this hypothesis, obtaining a $\Gamma_{3}\left(\Gamma_{4}\right)$ ground state (first excited state) split by $\sim 30 \mathrm{~K}$ [383]. The measured crystal-field excitations are very clear, indicating there are well-localized $4 f$ electron states that have not been broadened by correlation effects as typically occurs in Kondo systems [383]. This observation suggests that any heavy-fermion behavior in $\operatorname{PrIr}_{2} \mathrm{Zn}_{20}$ must originate from 
nonmagnetic degrees of freedom. The $\Gamma_{3}$ ground state carries nonmagnetic quadrupolar degrees of freedom, and antiferroquadrupolar order is observed at $T_{Q} \simeq 0.11 \mathrm{~K}$ [382]; superconductivity emerges from within this ordered state $\left(T_{c}<T_{Q}\right)$ [382]. The entropy released at $T_{Q}$ is only $0.2 R \ln 2$, indicating that quadrupolar fluctuations could play an important role in the formation of Cooper pairs [382]. However, extremely low upper critical field values with upper limit $H_{c 2}(0)<20$ Oe are observed and cyclotron effective masses of order $m_{0}$ are obtained in quantum oscillation measurements [384], indicating that Cooper pairs are not formed from heavy quasiparticles in $\operatorname{PrIr}_{2} \mathrm{Zn}_{20}$. Though it is probably not a heavy-fermion compound, uncovering superconductivity in $\operatorname{PrIr}_{2} \mathrm{Zn}_{20}$ (and in the related compound $\operatorname{PrRh}_{2} \mathrm{Zn}_{20}$ at $T_{c} \simeq 0.06 \mathrm{~K}$ [385]) set the stage for the discovery of heavy-fermion superconductivity in other Pr-based 1-2-20 compounds with $\Gamma_{3}$ ground states.

A nonmagnetic non-Kramers $\Gamma_{3}$ doublet ground state is also observed in the compound $\operatorname{PrTi}_{2} \mathrm{Al}_{20}$. The well-localized quadrupoles associated with this $\Gamma_{3}$ ground state order at $T_{Q} \simeq$ $2.0 \mathrm{~K}$ [259]. A large feature is observed at $T_{Q}$ in the specific heat and a corresponding entropy of nearly $R \ln 2$ is recovered at $T_{Q}$; the low-temperature entropy and $\chi(T)$ behavior are consistent with a $\Gamma_{3}$ ground state [259]. The specific heat feature at $T_{Q}$ broadens under applied magnetic field, suggesting that order is possibly ferroquadrupolar in nature [259]. This hypothesis was confirmed in ultrasound measurements of $\operatorname{PrTi}_{2} \mathrm{Al}_{20}$ that directly probe the quadrupolar susceptibility [386]. CurieWeiss behavior is observed in $\chi(T)$ data for $T>250 \mathrm{~K}$, characterized by $\Theta_{C W} \simeq-40 \mathrm{~K}$ and $\mu_{\text {eff }} \simeq 3.43 \mu_{B} / \operatorname{Pr}$ [259]. Such a large $\Theta_{C W}$ could not be associated with the RKKY energy scale and must instead characterize a Kondo energy scale associated with the excited multiplet states. At lower temperature, a Kondo resonance is observed at $\epsilon_{F}$ in resonant photoemission spectroscopy measurements [387], and the $\rho(T) \sim T^{2}$ behavior for $T_{Q} \lesssim T \lesssim 20 \mathrm{~K}$ and enhanced value of the Sommerfeld coefficient $\gamma \simeq 100 \mathrm{~mJ} \mathrm{~mol}^{-1} \mathrm{~K}^{-2}$ all suggest that $\operatorname{PrTi}_{2} \mathrm{Al}_{20}$ is a moderately-heavy Fermi liquid system [260]. This interpretation is supported by estimating the Kadowaki-Woods ratio $A / \gamma^{2} \simeq 3.3 \times 10^{-6} \mu \Omega \mathrm{cm} \mathrm{K}^{2} \mathrm{~mol}^{2} \mathrm{~mJ}^{-2}$ [260], which is comparable to the quasi-universal values exhibited by heavy Fermiliquid systems [20, 21].

Superconductivity with $T_{c} \simeq 0.2 \mathrm{~K}$ emerges in $\operatorname{PrTi}_{2} \mathrm{Al}_{20}$ at ambient pressure out of this ferroquadrupolar ordered state [260]. A volume fraction of $\sim 60 \%$ was obtained in zerofield cooled measurements of $\chi(T)$, which suggests that superconductivity is bulk [260]. The upper critical field value was measured with $H \|$ [110] in this cubic compound to be $H_{c 2}(0) \simeq 6 \mathrm{mT}$ [260]. The upper critical field curve $H_{c 2}(T)$ agrees with a calculation from WHH theory for orbital limiting except near $T_{c}$ where the experimentally measured initial slope $\left(d H_{c 2} / d T\right)_{T_{c}}$ is slightly smaller than the best-fit value $\left(d H_{c 2} / d T\right)_{T_{c}} \simeq-47 \mathrm{mT} / \mathrm{K}$ [260]; this discrepancy could be related to a multigap scenario for superconductivity in $\operatorname{PrTi}_{2} \mathrm{Al}_{20}$ [260]. The WHH theory predicts $H_{c 2}^{*}(0) \simeq 6.3 \mathrm{mT}$, which agrees much better with the measured $H_{c 2}(0)$ value than the estimated Pauli paramagnetic limit of $H_{p 0} \simeq 335 \mathrm{mT}$ [260]. From analysis of the $H_{c 2}(T)$ curve, the effective quasiparticle mass is estimated to be $m^{*} / m_{0} \sim 16$, which is consistent with a moderate mass enhancement [260].

An effort was made to enhance the hybridization strength in $\operatorname{PrTi}_{2} \mathrm{Al}_{20}$ and to induce a quadrupolar Kondo effect by applying pressure [31]. With increasing pressure, $T_{Q}(P)$ traces out a weak dome with maximum near $6 \mathrm{GPa}$ and superconductivity emerges above 6.7 $\mathrm{GPa}$ [31]; the observed dome of superconductivity has maximum $T_{c} \simeq 1.1 \mathrm{~K}$ at $8.7 \mathrm{GPa}$ [31]. This pressure-induced superconducting state is distinct from the superconductivity observed at ambient pressure $\left(T_{c} \simeq 0.2 \mathrm{~K}\right)$ and emerges from a much heavier Fermi-liquid state [31]. These phases can be seen in Fig. 14(a). Bulk superconductivity in $\operatorname{PrTi}_{2} \mathrm{Al}_{20}$ was suggested by a large diamagnetic response in measurements of ac magnetic susceptibility and confirmed by a jump at $T_{c}$ in the specific heat under applied pressure [31]. At 8.7 GPa, the upper critical field is $H_{c 2}(0)>3 \mathrm{~T}$ with a large initial slope $\left(d H_{c 2} / d T\right)_{T_{c}} \simeq-6.0 \mathrm{~T} / \mathrm{K}$ (see Fig. 14(b)) [31]. The orbital limiting field is estimated using WHH theory [59] to be $H_{c 2}^{*}(0) \simeq 4.7 \mathrm{~T}$ [31], which exceeds the Pauli limit of $H_{p 0} \simeq$ $2 \mathrm{~T}$. Using the upper critical field values at $8.7 \mathrm{GPa}$, an effective mass of $m^{*} / m_{0} \sim 106$ is estimated [31], unambiguously confirming the heavy-fermion character of superconductivity in $\operatorname{PrTi}_{2} \mathrm{Al}_{20}$ under applied pressure. The electrical resistivity in the normal state exhibits a robust $T^{3}$ temperature dependence that may be a consequence of fluctuations of quadrupolar order [31]. It is possible that the heavy-fermion superconductivity in $\mathrm{PrTi}_{2} \mathrm{Al}_{20}$ may emerge in the vicinity of a quantum critical point associated with the suppression of ferroquadrupolar order where strong fluctuations arise due to competition between quadrupolar order and the quadrupolar Kondo effect [31].

$\operatorname{PrV}_{2} \mathrm{Al}_{20}$ is another 1-2-20 compound that exhibits a nonmagnetic $\Gamma_{3}$ non-Kramers doublet ground state. In contrast to $\operatorname{PrTi}_{2} \mathrm{Al}_{20}$, stronger hybridization in $\operatorname{PrV}_{2} \mathrm{Al}_{20}$ leads to heavyfermion behavior at ambient pressure. Quadrupolar order is observed at $T_{Q} \simeq 0.6 \mathrm{~K}$ that is sensitive to sample purity [259]; two clear and distinct transitions are observed at $T_{Q} \simeq 0.75 \mathrm{~K}$ and $T^{*} \simeq 0.65$ in samples with residual resistivity ratio (RRR) $>7$, leading to speculations regarding the possibility of order from octapolar degrees of freedom as well [62]. A broad feature is observed in the specific heat of $\operatorname{PrV}_{2} \mathrm{Al}_{20}$ and the corresponding entropy is found to be $\sim(R / 2) \ln 2$ at $T_{Q}[259,62]$. The response of this feature to applied magnetic field is consistent with antiferroquadrupolar order [259]. Curie-Weiss analysis of $\chi(T)$ data at high temperature yields $\Theta_{C W} \simeq-55 \mathrm{~K}$ with $\mu_{\text {eff }} \simeq 3.57 \mu_{B} / \operatorname{Pr}$ [259]. A large Kondo energy scale is implied by the value of $\Theta_{C W}$. Non-Fermi liquid behavior is observed for $T>T_{Q}$ in the low-temperature physical properties including $C / T \sim T^{-3 / 2}, \chi(T) \sim-T^{1 / 2}$, and $\rho(T) \sim T^{1 / 2}$ temperature dependencies [259]. These behaviors are consistent with predictions for the two-channel or quadrupolar Kondo effect [27, 28, 29], and this possibility is strengthened by the $(R / 2) \ln 2$ entropy released at $T_{Q}$ [62]. The $\rho(T) \simeq T^{1 / 2}$ behavior was observed in samples with a wide-range of purity, indicating that this state is robust (i.e., in contrast to superconductivity) [259]. Partial quenching of the quadrupole moment is manifested in the feature in the specific heat at $T_{Q}$ which is weaker than the corresponding feature in $\operatorname{PrTi}_{2} \mathrm{Al}_{20}$. The Som- 

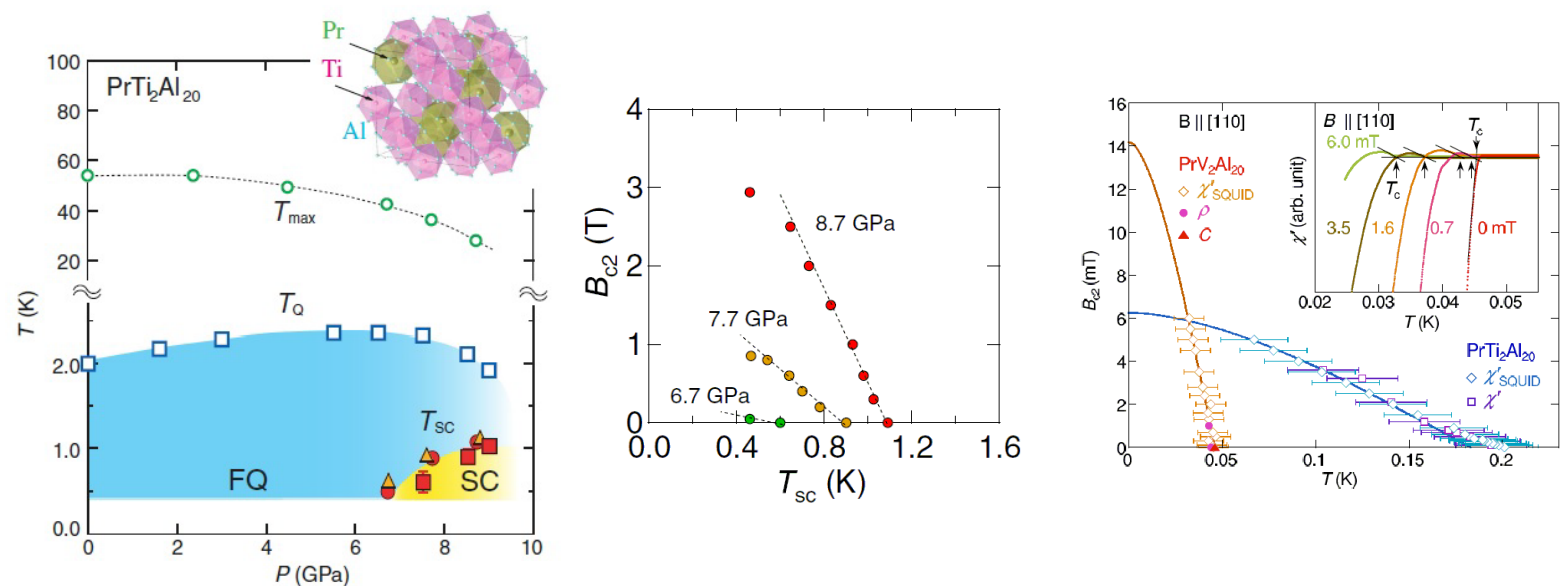

Figure 14: (Color online) (a) Temperature-pressure $(T-P)$ phase diagram for $\operatorname{PrTi}_{2} \mathrm{Al}_{20}$. Unfilled circles and squares represent $T_{\text {max }}$, determined from the maxima in electrical resistivity measurements, and the ferroquadrupole (FQ) ordering temperature $T_{Q}$, respectively. A dome of pressure-induced superconductivity (SC) is observed with maximum near $9 \mathrm{GPa}$ and the $T_{c}$ values are obtained from electrical resistivity (filled circles), ac magnetic susceptibility (filled triangles), and specific heat (filled squares) measurements, respectively. The inset displays the cubic crystal structure of $\operatorname{PrTi}_{2} \mathrm{Al}_{20}$. (b) $\mathrm{The}_{\mathrm{p}}$ uper critical fields $H_{c 2}(T)$ for $\operatorname{PrTi}_{2} \mathrm{Al}_{20}$ under applied pressures of $6.7 \mathrm{GPa}, 7.7 \mathrm{GPa}$, and $8.7 \mathrm{GPa}$, where $T_{c}$ was determined from the temperature where the electrical resistivity vanishes. The increase of $\left(d H_{c 2} / d T\right)_{T_{c}}$ with increasing pressure is dramatic. (c) $H_{c 2}(T)$ for single crystals of $\operatorname{PrV}_{2} \mathrm{Al}_{20}$ and $\operatorname{PrTi}_{2} \mathrm{Al}_{20}($ at ambient pressure) with $H \|[110]$. The square, diamond, circle, and triangle data points are determined from measurements of ac-susceptibility, ac-susceptibility with a SQUID, electrical resistivity, and specific heat, respectively. The solid lines represent fits based on the WHH model [59] for the orbital critical field. The inset displays the real part of the ac-susceptibility for $\mathrm{PrV}_{2} \mathrm{Al}_{20}$ under various magnetic fields and the arrows indicate $T_{c}$ values. Panels (a) and (b) are adapted from Ref. [31] and panel (c) is from Ref. [62].

merfeld coefficient value is $\gamma \simeq 90 \mathrm{~mJ} \mathrm{~mol}^{-1} \mathrm{~K}^{-2}$ [62], which is modestly enhanced and consistent with the relatively strong hybridization in $\operatorname{PrV}_{2} \mathrm{Al}_{20}$.

From this anitferroquadrupolar ordered heavy-fermion state, superconductivity is observed at ambient pressure with $T_{c} \simeq$ $0.05 \mathrm{~K}$ in samples with high purity (RRR 19) [62]. Superconductivity is not observed in samples with $\mathrm{RRR}<5$, suggesting that superconductivity in $\operatorname{PrV}_{2} \mathrm{Al}_{20}$ is unconventional [62]. Magnetic susceptibility measurements indicate superconducting volume fractions are as large as $82 \%$ and a jump in the specific heat at $T_{c}$ of $\Delta C / \gamma T_{c} \simeq 0.3$ [62] both act to confirm the bulk nature of superconductivity in $\operatorname{PrV}_{2} \mathrm{Al}_{20}$. The upper critical field at zero temperature is estimated to be $H_{c 2}(0) \simeq$ $14 \mathrm{mT}$ with an initial slope of $\left(d H_{c 2} / d T\right)_{T_{c}} \simeq-0.41 \mathrm{~T} / \mathrm{K}$ (see Fig. 14(c)), and it appears to follow a $H_{c 2}(T)$ curve calculated using WHH theory [62]. This observation indicates that Cooper pairs are probably orbitally limited. The value of $\left(d H_{c 2} / d T\right)_{T_{c}}$ for $\operatorname{PrV}_{2} \mathrm{Al}_{20}$ is an order of magnitude larger than the corresponding value in $\operatorname{PrTi}_{2} \mathrm{Al}_{20}$ at ambient pressure [260], indicating that quasiparticles have a significantly heavier effective mass. An enhanced effective mass of $m^{*} / m_{0} \sim 140$ for $\mathrm{PrV}_{2} \mathrm{Al}_{20}$ is estimated using the upper critical field [62], which is comparable to that of $\operatorname{PrTi}_{2} \mathrm{Al}_{20}$ under an applied pressure near $8 \mathrm{GPa}$ [31]. This similarity suggests that $\mathrm{PrV}_{2} \mathrm{Al}_{20}$ may also be in close proximity to a quantum critical point associated with quadrupolar order [62]. If this hypothesis is correct, the pairing mechanism for superconductivity in $\operatorname{PrTi}_{2} \mathrm{Al}_{20}$ and $\operatorname{PrV}_{2} \mathrm{Al}_{20}$ is probably the same and is likely associated with quadrupolar fluctuations.

This family of compounds that adopt the $\mathrm{CeCr}_{2} \mathrm{Al}_{20}$-type crystal structure has been a fertile reservoir of new Pr-based heavy-fermion superconductors so far. The exciting recent discovery of Cd-based 1-2-20 compounds [388, 389] includes $\mathrm{PrNi}_{2} \mathrm{Cd}_{20}$ and $\operatorname{PrPd}_{2} \mathrm{Cd}_{20}$, which are currently being studied to ascertain whether heavy-fermion superconductivity associated with potential $\Gamma_{3}$ ground state [390] might also be observed. Regardless of the outcome of those studies, it appears that the point-group symmetry of the crystallographic site filled by the Pr ion and the other properties arising from the crystal structure, make the 1-2-20 compounds an excellent place to search for new Pr-based heavy-fermion superconductors.

\subsection{Yb-based compound: $\beta$-YbAlB 4}

The trivalent $\mathrm{Yb}$ ion has a $4 f^{13}$ electronic configuration, and is therefore often considered to be the hole analogue of trivalent Ce with its $4 f^{1}$ electronic configuration. Naively, this similarity combined with the propensity for valence instability would be expected to produce similar Kondo physics in $\mathrm{Yb}$ compounds; yet, the number of Yb-based heavy-fermion compounds is far smaller than the number of Ce-based heavy-fermion compounds. This interesting empirical fact was discussed by Fisk and Maple who, based on the single Ce-based heavy-fermion superconductor known at the time $\left(\mathrm{CeCu}_{2} \mathrm{Si}_{2}\right)$, speculated that there would not be many $\mathrm{Yb}$-based heavy-fermion compounds that also exhibit superconductivity [391]. This prediction has so far been validated: only a single Yb-based heavy-fermion superconductor has been reported to date.

Superconductivity was observed in 2008 in high-purity samples (RRR 300) of the heavy-fermion compound $\beta$-YbAlB 4 with a $T_{c} \simeq 0.08 \mathrm{~K}[63,261]$. This compound forms with an orthorhombic crystal structure, characterized by the space group Cmmm [63]. The crystal structure is composed of $\mathrm{Yb}$ 
and $\mathrm{Al}$ ions sandwiched between $\mathrm{B}$ layers, which might be expected to produce a two-dimensional Fermi surface; however, local density approximation (LDA) band-structure calculations [392] and quantum oscillation studies [393] demonstrate that $\beta$-YbAlB 4 has a three-dimensional Fermi surface composed of two sheets from heavy bands of $f$-like character.

We begin our discussion of $\beta$-YbAlB 4 by describing its normal-state properties. Calculations suggest that $\beta$-YbAlB 4 should exhibit an antiferromagnetic ground state, however, it remains paramagnetic instead [392]. Magnetic susceptibility $\chi(T)$ measurements demonstrate strong uniaxial anisotropy that is consistent with an Ising system with magnetic moments tending to align along the $c$ axis [63]. With $H \| c$, Curie-Weiss behavior in $\chi(T)$ data is observed for $T>100 \mathrm{~K}$ with $\Theta_{C W} \sim-210$ $\mathrm{K}$ and an effective Ising moment of $\sim 3.1 \mu_{B}$; in contrast, the in-plane magnetic susceptibility is only weakly temperaturedependent with a subtle peak near $200 \mathrm{~K}$ [63]. The electrical resistivity $\rho(T)$ displays a weak coherence peak near $250 \mathrm{~K}$ and goes as $\rho \propto T$ for $1<T<4 \mathrm{~K}$ and $\rho \propto T^{1.5}$ for $T \lesssim 1 \mathrm{~K}$ [63]. These are strong manifestations of non-Fermi liquid behavior in $\beta-\mathrm{YbAlB}_{4}$. The features in $\chi(T)$ and $\rho(T)$ data near $200 \mathrm{~K}$ and $250 \mathrm{~K}$, respectively, reveal a relatively large Kondo energy scale [63]; however, careful measurements of the Hall effect in $\beta$-YbAlB 4 later provided evidence for two distinct Kondo-like energy scales at $40 \mathrm{~K}$ and $200 \mathrm{~K}$ [394]. The Sommerfeld coefficient can be estimated by applying a small magnetic field, causing the diverging $C / T$ data at low temperature to saturate; a value of $\gamma \simeq 150 \mathrm{~mJ} \mathrm{~mol}^{-1} \mathrm{~K}^{-2}$ is obtained by this procedure [63]. An effective mass of the quasiparticles $m^{*} / m_{0} \sim 180$ can be estimated [261] while values of $m^{*} / m_{0} \sim 5-14$ are obtained in Shubnikov-de Haas experiments [393]. These latter values of $m^{*} / m_{0}$ have been suppressed by the high magnetic fields used in the quantum oscillation measurements as we discuss below.

Astoundingly, the intriguing non-Fermi liquid state observed in the physical properties at low temperature [63] is accessed in $\beta$-YbAlB 4 without any external tuning [395]. Furthermore, this behavior is apparently robust [63], being observed in samples with RRR values between 75-300 including samples that do not exhibit superconductivity [261]. Fermi-liquid behavior is rapidly restored in $\beta-\mathrm{YbAlB}_{4}$ by applying a magnetic field, with the $\rho \propto T^{1.5}$ behavior only being observed in early experiments when $H<0.1 \mathrm{~T}$ [63]; this upper limit for the crossover field was later reduced to $0.2 \mathrm{mT}$ [395]. By fitting the Fermiliquid $\rho \simeq A T^{2}$ behavior in several magnetic fields $(H \| c)$, it is observed that $A \sim H^{1 / 2}$, which is consistent with the presence of a quantum critical point at zero-field [63]. Furthermore, unconventional quantum criticality is manifested in $T / H$ scaling of the thermodynamic free energy over a wide range of temperatures and magnetic fields (see Fig. 15) and a diverging effective quasiparticle mass $m^{*} \sim H^{-1 / 2}$ [395]. When these unconventional signatures of quantum criticality are combined with the intermediate $\mathrm{Yb}$ valence in $\beta$-YbAlB 4 (discussed below), the zero-field quantum critical point must be unconventional as well [395]. A model to describe the intrinsic quantum criticality observed in $\beta$-YbAlB 4 has been proposed by Ramires et al. [396].

The valence of $\mathrm{Yb}$ in $\beta$ - $\mathrm{YbAlB}_{4}$ was initially predicted to be

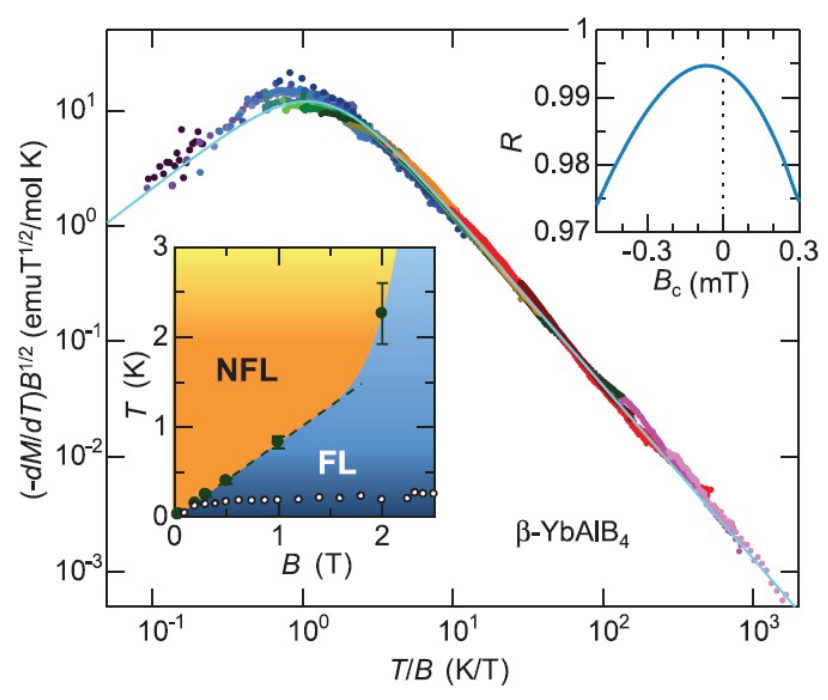

Figure 15: (Color online) Scaling of the thermodynamic free energy of $\beta$ $\mathrm{YbAlB}_{4}$, represented by $-(d M / d T) B^{1 / 2}$ as obtained from experimental measurements of magnetic susceptibility $M$, over several decades of the parameter $T / B$ (where $T$ is temperature and $B$ is magnetic field). This scaling suggests that a quantum critical point (QCP) is found at zero field. If a QCP exists at $B_{c} \neq 0 \mathrm{~T}$, the free energy will scale with a parameter $T /\left|B-B_{c}\right|$. The best fits of $-(d M / d T) B^{1 / 2}$ using this more generalized scaling parameter place an upper bound of $\left|B_{c}\right|<0.2 \mathrm{mT}$ as is seen in the upper inset showing Pearson's correlation coefficient $R$ vs. $B_{c}$. The lower left inset displays the magnetic field-temperature $(B-T)$ phase diagram of $\beta$-YbAlB 4 . The filled circles are determined from the peak temperatures in $-d M / d T$, and denote a crossover between non-Fermi liquid and Fermi liquid behavior. The dashed line indicates $k_{B} T \sim g \mu_{B} B$. The unfilled circles depict the temperatures, below which, the electrical resistivity shows a quadratic temperature dependence (after Ref. [395]).

intermediate in LDA calculations [392], and hard x-ray photoemission measurements subsequently observed signatures of an intermediate $\mathrm{Yb}$ valence in the $\mathrm{Yb} 3 d$ spectra [397]. From measurements at $20 \mathrm{~K}$, the $\mathrm{Yb}$ valence was determined to be $\mathrm{Yb}^{2.75(2)+}$, which is much lower than the $2.9+$ to $3+\mathrm{Yb}$ valences typically observed in other Yb-based heavy-fermion compounds [397]. This intermediate $\mathrm{Yb}$ valence, which is a consequence of strong hybridization between $4 f$ and conduction electron states, is consistent with the relatively large Kondo energy scale of order 200-250 K [395]. The valence fluctuations in $\beta-\mathrm{YbAlB}_{4}$ are expected to play a role in producing the non-Fermi liquid behavior, which is intriguingly similar to the behavior of the intermediate valence materials $\mathrm{YbRh}_{2} \mathrm{Si}_{2}$ and $\mathrm{YbRh}_{2}\left(\mathrm{Si}_{0.95} \mathrm{Ge}_{0.05}\right)_{2}$ [398]. Adding credibility to this possibility, an electron spin resonance (ESR) signal is observed in $\beta$-YbAlB 4 [399] that is similar to ESR results for $\mathrm{YbRh}_{2} \mathrm{Si}_{2}$, begging the question of whether there is a connection between this ESR signal and quantum criticality in these intermediate valence compounds [399, 400]. While each compound exhibits a similar diverging Nernst coefficient in the vicinity of their respective quantum critical points, the response of the ratio of thermoelectric power divided by temperature, $S / T$, and $\gamma$ for each is different [401]. This latter result suggests that comparisons between these two compounds need to be made carefully, but the present observations offer intriguing support for a rela- 
tionship between valence fluctuations and the non-Fermi liquid behavior in $\beta$-YbAlB 4 .

Superconductivity in $\beta$ - $\mathrm{YbAlB}_{4}$ emerges from this nonFermi liquid state with a $T_{c}$ that depends sensitively on sample purity (superconductivity is only present in samples with RRR > 100) [63]. This sensitivity to disorder is consistent with an unconventional superconductivity with non- $s$-wave character [63]. Measurements of the magnetization in a $0.1 \mathrm{mT}$ magnetic field obtained a superconducting volume fraction of $15 \%$ $45 \%$ in zero-field cooled measurements $[63,261]$. The reduced values of the volume fraction are partially related to the long magnetic penetration depth relative to the small dimensions of the sample [261]. To date, a jump in the specific heat has not been reported for $\beta$-YbAlB 4 , but these magnetic susceptibility results may be consistent with bulk superconductivity.

The upper critical fields $H_{c 2}(T)$ of $\beta-\mathrm{YbAlB}_{4}$ are anisotropic with an anomalous suppression at low temperature that is likely associated with paramagnetic effects related to the $c$-axis magnetic susceptibility [261]. The initial slopes are measured to be $\left(d H_{c 2}^{a} / d T\right)_{T_{c}} \simeq-2.6 \mathrm{~T} / \mathrm{K}$ and $\left(d H_{c 2}^{c} / d T\right)_{T_{c}} \simeq-1.4 \mathrm{~T} / \mathrm{K}$ with extrapolated values at zero temperature of $H_{c 2}^{a}(0) \simeq 0.15 \mathrm{~T}$ and $H_{c 2}^{c}(0) \simeq 0.025 \mathrm{~T}$ [261]. An estimate for the orbital critical field yields $H_{c 2}^{*, a}(0) \simeq 0.15 \mathrm{~T}$, which is in excellent agreement with the observed results for $H_{c 2}^{a}(0)$ [261]. However, $H_{c 2}^{c}(0)<H_{c 2}^{*, c} \simeq 0.076 \mathrm{~T}<H_{p 0} \simeq 0.15 \mathrm{~T}$, indicating the presence of other pair-breaking effects beyond simple orbital and paramagnetic effects [261]. Superconducting coherence lengths at zero temperature of $\xi_{a}(0) \simeq 66 \mathrm{~nm}$ and $\xi_{c}(0) \simeq 33 \mathrm{~nm}$ are estimated using the Ginzburg-Landau formula for an anisotropic superconductor [261]. In a sample that did not exhibit superconductivity above $30 \mathrm{mK}$, the mean free path is only a factor of 4 times larger than $\xi_{a}(0)$, indicating that superconductivity in $\beta$-YbAlB 4 only appears in the clean limit [261].

\subsection{Pu- and Np-based heavy-fermion superconductors}

Around the same time as the discovery of the first heavyfermion compound, $\mathrm{CeAl}_{3}$ [9], it was recognized that a quadratic temperature dependence of the electrical resistivity at low temperatures and large values of the Sommerfeld coefficient $\gamma$ could be found in transuranium compounds like $\mathrm{PuAl}_{2}$ [402, 403]. These early studies demonstrated that such compounds could exhibit heavy Fermi-liquid states; though, this was not completely appreciated at the time. Superconductivity at ambient pressure was discovered in transuranium compounds with $T_{c}=18.5 \mathrm{~K}$ in PuCoGa 5 [107], $T_{c}=8.7 \mathrm{~K}$ in PuRhGa [108], and $T_{c}=4.9 \mathrm{~K}$ in $\mathrm{NpPd}_{5} \mathrm{Al}_{2}$ [221]. The properties of these compounds have been reviewed in Refs. [404, 111, 262] and the $\mathrm{Pu}$-based systems are also addressed in a dedicated article by Sarrao et al. in this special issue of Physica C. Therefore, we will only briefly summarize the highlights. Along with compounds like $\mathrm{U}_{6} \mathrm{~T}$ ( $\mathrm{T}=$ transition metal), these compounds may constitute a link that bridges traditional Ce and U-based heavyfermion superconductors and other classes of unconventional superconductors including the cuprates.

The compounds $\mathrm{PuCoGa}_{5}$ and $\mathrm{PuRhGa}_{5}$ crystallize in a tetragonal crystal structure characterized by space group
$P 4 / \mathrm{mmm}$ and are isostructural with the Ce-based " 115 " compounds discussed in Section 2.2. These compounds exhibit comparable, modestly-enhanced Sommerfeld coefficient values of $\gamma \simeq 77 \mathrm{~mJ} \mathrm{~mol}^{-1} \mathrm{~K}^{-2}$ and $70 \mathrm{~mJ} \mathrm{~mol}^{-1} \mathrm{~K}^{-2}$ for PuCoGa 5 and PuRhGa , respectively [107, 262]. These values can be compared to $\gamma \simeq 50 \mathrm{~mJ} \mathrm{~mol}^{-1} \mathrm{~K}^{-2}$ for $\delta$-Pu [107]. Bulk superconductivity is demonstrated by the jumps observed at $T_{c}$ in the specific heat measurements. For PuCoGa 5 , a jump of $\Delta C / \gamma T_{c} \simeq 1.4$ [107] is very close to the BCS value, while a smaller jump of $\Delta C / \gamma T_{c} \simeq 0.65$ is observed for $\mathrm{PuRhGa}_{5}$ [264]. In the superconducting state of each compound, the quadratic temperature dependence of the specific heat provides evidence for line nodes in the superconducting energy gap [264, 263]. The initial slope of the upper critical field of $\mathrm{PuCoGa}_{5}$ is $\left(d H_{c 2} / d T\right)_{T_{c}} \simeq-5.9 \mathrm{~T} / \mathrm{K}$, which implies an enormous $H_{c 2}^{*}(0) \simeq 74 \mathrm{~T}$ [107]; measurements of the initial slope using measurements of specific heat suggest even larger values of $\left(d H_{c 2}^{a} / d T\right)_{T_{c}} \simeq-10 \mathrm{~T} / \mathrm{K}$ and $\left(d H_{c 2}^{c} / d T\right)_{T_{c}} \simeq-8 \mathrm{~T} / \mathrm{K}$ [264]. For $\mathrm{PuRhGa}_{5}$, the initial slopes of the upper critical fields are smaller at $\left(d H_{c 2}^{a} / d T\right)_{T_{c}} \simeq-3.5$ to $-4 \mathrm{~T} / \mathrm{K}$ and $\left(d H_{c 2}^{c} / d T\right)_{T_{c}} \simeq$ $-2 \mathrm{~T} / \mathrm{K}$ [264, 262]. The values of the upper critical at zero temperature for $\mathrm{PuRhGa}_{5}$ are measured to be $H_{c 2}^{a}(0) \simeq 27 \mathrm{~T}$ and $H_{c 2}^{c}(0) \simeq 15 \mathrm{~T}$, which are comparable to estimates of the orbital-limiting fields [262]. Superconductivity in these compounds degrades with time due to self-irradiation effects. For example, the rate of change $\Delta T_{c} / \Delta t$ is $\sim-0.24 \mathrm{~K} / \mathrm{month}$ and $\sim$ $0.39 \mathrm{~K} /$ month for $\mathrm{PuCoGa}_{5}$ and $\mathrm{PuRhGa}_{5}$, respectively [49].

The compound $\mathrm{NpPd}_{5} \mathrm{Al}_{2}$ crystallizes with the tetragonal crystal structure with space group $I 4 / \mathrm{mmm}$. Like the Pu-based superconductors, $\mathrm{NpPd}_{5} \mathrm{Al}_{2}$ is paramagnetic with an enhanced Sommerfeld coefficient of $\gamma \simeq 200 \mathrm{~mJ} \mathrm{~mol}^{-1} \mathrm{~K}^{-2}$ [221]. In the normal state, non-Fermi liquid behavior is observed as manifested by a linear temperature dependence of the electrical resistivity, followed by a transition to superconductivity at $T_{c} \simeq$ $4.9 \mathrm{~K}$ [221]. At $T_{c}$, a jump of $\Delta C / \gamma T_{c} \simeq 2.33$ is observed in the specific heat, confirming the bulk nature of strongly-coupled superconductivity [221]. For $T<T_{c}$, the cubic temperature dependence of the specific heat suggests the presence of point nodes in the superconducting energy gap structure [221]. The strongly-anisotropic initial slopes of the upper critical fields are $\left(d H_{c 2}^{a} / d T\right)_{T_{c}} \simeq-6.4 \mathrm{~T} / \mathrm{K}$ and $\left(d H_{c 2}^{c} / d T\right)_{T_{c}} \simeq-31 \mathrm{~T} / \mathrm{K}$, and the measured values of the upper critical fields of $\mathrm{NpPd}_{5} \mathrm{Al}_{2}$ at zero temperature are $H_{c 2}^{a}(0) \simeq 3.7 \mathrm{~T}$ and $H_{c 2}^{c}(0) \simeq 14 \mathrm{~T}$ [221]. This value of $H_{c 2}^{c}(0)$ is strongly paramagnetically-limited $\left(H_{p 0} \simeq 9\right.$ T) relative to the expected orbital critical field of $H_{c 2}^{*, c}(0) \simeq 105$ $\mathrm{T}$ [221].

\section{Concluding remarks}

\subsection{General characteristics of superconductivity in heavy- fermion compounds}

Throughout this selective review, we have frequently encountered the same, or strikingly similar, properties in the superconducting and normal states of the of heavy-fermion compounds that we have discussed. These characteristics, many of which are presented in Table 3, constitute a basic set of properties that 
are shared by all superconducting heavy-fermion compounds. They can be divided into six properties that we briefly describe below.

(1) The superconducting transition temperatures $T_{c}$ are generally of order $1 \mathrm{~K}$ with some being as low as $0.05 \mathrm{~K}$ (like in $\operatorname{PrV}_{2} \mathrm{Al}_{20}$ [62]) and some as high as 2.3-2.4 $\mathrm{K}$ (like in $\mathrm{CeCoIn}_{5}$ [96] and $\mathrm{CeRhIn}_{5}$ [94] under ambient and applied pressures, respectively). In this review, we have also briefly discussed a few compounds with $T_{c}$ values that are significantly higher, including $\mathrm{U}_{6} \mathrm{Fe}\left(T_{c} \simeq 3.8 \mathrm{~K}\right)$ [225] and the actinidebased compounds $\mathrm{PuCoGa}_{5}\left(T_{c} \simeq 18.5 \mathrm{~K}\right)$ [107], $\mathrm{PuRhGa}_{5}$ $\left(T_{c} \simeq 8.7 \mathrm{~K}\right)$ [108], and $\mathrm{NpPd}_{5} \mathrm{Al}_{2}\left(T_{c} \simeq 4.9 \mathrm{~K}\right)$ [221]. However, despite sharing many compelling similarities with superconducting heavy-fermion compounds, these latter compounds may rather be links that connect the heavy-fermion compounds to the cuprates and other classes of unconventional superconductors.

(2) The specific heat jumps at $T_{c}$ in heavy-fermion compounds are of order $\gamma T_{c}$. Since heavy-fermion compounds are characterized by significantly-enhanced values of $\gamma$, this result reveals that it is the heavy quasiparticles that pair in their superconducting states. The measured $\Delta C / \gamma T_{c}$ values typically range between $0.2-4.5$ (see Table 3 ). Unfortunately, there are many compounds for which $\Delta C / \gamma T_{c}$ has not yet been measured because of experimental challenges associated with measuring specific heat at either extremely low temperature (i.e., when $T_{c}$ values are very low) or under applied pressure (i.e., when superconductivity is pressure-induced).

(3) The measured upper critical fields $H_{c 2}(T)$ in heavyfermion compounds have very large initial slopes $\left(d H_{c 2} / d T\right)_{T_{c}}$ near $T_{c}$ (see Table 2 for some examples). These values of $\left(d H_{c 2} / d T\right)_{T_{c}}$ are generally on the order $-1 \mathrm{~T} / \mathrm{K}$ to $-10 \mathrm{~T} / \mathrm{K}$ with particularly high values for $\mathrm{CeCu}_{2} \mathrm{Si}_{2}(-23 \mathrm{~T} / \mathrm{K})$ [66], $\mathrm{CeRhSi}_{3}$ $(-23 \mathrm{~T} / \mathrm{K}$ at $2.6 \mathrm{GPa})$ [213], $\mathrm{CeCoIn}_{5}(-24 \mathrm{~T} / \mathrm{K}$ for $H \| a b)$ [133], and $\mathrm{UBe}_{13}(-42 \mathrm{~T} / \mathrm{K})$ [347]. Such large $\left(d H_{c 2} / d T\right)_{T_{c}}$ values indicate that the orbital upper critical fields at zero temperature, $H_{c 2}^{*}(0)$, are also quite large; as a consequence, superconductivity in many heavy-fermion compounds is paramagnetically limited (i.e., $H_{c 2}(0) \simeq H_{p 0}<H_{c 2}^{*}(0)$ ).

(4) The large values of $\left(d H_{c 2} / d T\right)_{T_{c}}$ and $H_{c 2}^{*}(0)$ in heavyfermion compounds also indicate that the Ginzburg-Landau coherence lengths at zero temperature, $\xi(0)$, tend to be short; $\xi(0)$ values of order $10-100 \AA$ are typical. Such short coherence lengths are consistent with the heavy effective masses, $m^{*}$, of the quasiparticles involved in pairing, since $\xi(0) \propto 1 / m^{*}$. The Ginzburg-Landau parameter, which is the ratio of the London penetration depth $\lambda$ to $\xi$, is $\lambda / \xi>>1 / \sqrt{2}$ in heavy-fermion compounds, indicating that they are extreme type II superconductors.

(5) Superconductivity in heavy-fermion compounds is unambiguously unconventional. In the superconducting state, experimental evidence is consistent with quasiparticle pairs that are characterized by either $d$-wave, spin singlet $(L=2, S=$ 0 ) or $p$-wave, spin triplet $(L=1, S=1)$ states. Pairing in most of the heavy-fermion compounds is believed to be $d$ wave, but potential evidence for spin-triplet pairing has been observed in some compounds profiled in this article including
$\mathrm{CePt}_{3} \mathrm{Si}, \mathrm{CeIrSi}{ }_{3}, \mathrm{UNi}_{2} \mathrm{Al}_{3}, \mathrm{UGe}_{2}, \mathrm{UCoGe}$, and $\mathrm{U}_{2} \mathrm{PtC}_{2}$ (see their respective sections). This evidence generally comes from NMR measurements of a temperature-independent Knight shift and/or by measuring $H_{c 2}(0)$ values that dramatically exceed the Chandrasekhar-Clogston limit. The symmetry of the superconducting energy gap has not been established by phase-sensitive measurements in any of the heavy-fermion compounds as it has been in the cuprates [117], but point-contact [114] and tunneling spectroscopy $[115,116]$ measurements of $\mathrm{CeCoIn}_{5}$ are consistent with a $d_{x^{2}-y^{2}}$ gap symmetry. In nearly all of the heavyfermion compounds, power-law behavior in the temperature dependence of the specific heat, penetration depth, thermal conductivity, and other physical properties in the superconducting states are used to infer the existence of point or line nodes in the energy gaps [60]; additional information about the symmetry of $\Delta(\vec{k})$ and details concerning the nodal structure are sometimes obtained by studying modulations of the specific heat or thermal conductivity as a single crystal is rotated relative to a magnetic field with fixed orientation. The evidence from such studies on heavy-fermion compounds has been nearly universally consistent with anisotropic and nodal superconducting energy gaps; however, we note that evidence for nodeless gaps has been presented recently from measurements of the specific heat for $\mathrm{CeCu}_{2} \mathrm{Si}_{2}$ [75] and London penetration depth in the system $\mathrm{Ce}_{1-x} \mathrm{Yb}_{x} \mathrm{CoIn}_{5}[182,185]$. These results may force us to re-examine the conventional understanding of quasiparticle pairing in these systems and emphasize that there is still a lot of work remaining to obtain a comprehensive understanding of superconductivity in heavy-fermion compounds.

(6) The final characteristic that we will discuss is the proximity of the superconducting ground state to another ordered phase that tends to involve the magnetic or, less frequently, electric quadrupole degrees of freedom. These ordered phases are highly tunable, and in the case of heavy-fermion compounds, are susceptible to being suppressed by applied pressure or chemical substitution. This is evident in the significant number of heavy-fermion compounds within which superconductivity is pressure induced, emerging only after the ordered state has been suppressed. Such compounds have a superscripted asterisk following their $T_{c}$ values in Table 3 as well as an entry in the neighboring column to the right that provides the transition temperature of the magnetic or quadrupolar ordered phase at ambient pressure. For some compounds such as CeRhIn ${ }_{5}$, magnetic order need not be completely suppressed for superconductivity to emerge, and there are limited regions of phase space within which superconductivity and magnetic order coexist (see the phase diagram in Fig. 4 for example). When superconductivity is observed at ambient pressure, nature has already conveniently suppressed the nearby ordered phase. Compounds in this category exhibit an instability at ambient pressure towards ordering of the magnetic or quadrupole degrees of freedom. Sometimes the ordered phase that is on the verge of forming can be persuaded to emerge by modifying the chemical composition as in the spin-density wave order in $\mathrm{CeCu}_{2} \mathrm{Si}_{2}[66,70]$, exists at "negative pressure" like antiferromagnetic order in $\mathrm{CeCoIn}_{5}$, or is accessed by applying pressure like the antiferromagnetic or- 
der in $\mathrm{URu}_{2} \mathrm{Si}_{2}$ [304]. The abundance of spin (or quadrupolar) fluctuations that are found in these unstable environments are widely believed to play a role in mediating quasiparticle pairing in the superconducting state. Similarly, non-Fermi liquid behavior in the normal-state physical properties of many such heavy-fermion compounds is probably facilitated by the strong quantum fluctuations associated with the nearby quantum critical point $[15,8,39]$.

\subsection{Comparison of superconductivity in heavy-fermion com- pounds with that in the cuprates and iron pnic- tide/chalcogenide compounds}

Now that we have outlined and articulated some of the characteristics of the superconducting state in heavy-fermion compounds, we can briefly compare these properties with those of other classes of unconventional superconductors including the cuprates and iron pnictide/chalcogenide compounds. Many instructive review articles have been published that discuss the superconducting properties of the iron pnictide and iron chalcogenide compounds [405, 406, 407, 408] as well as the cuprates [409, 410, 411]; furthermore, there are articles dedicated to each of these classes of superconducting materials in this special issue of Physica C. Similarities between these families of unconventional superconductors, including the quasi-twodimensional layer components of their crystal structures, comparable phase diagrams, observations of a spin resonance in the superconducting states from inelastic neutron scattering measurements, and similar superconducting energy gap symmetries, have been discussed for some time [412, 204, 413]. We briefly address some of these similarities below.

The superconducting cuprates and iron pnictide/chalcogenide compounds are well-known for having layered crystal structures in which superconductivity resides within the $\mathrm{CuO}_{2}$ planes $[409,410,411]$ or corrugated $\mathrm{Fe} /$ pnictogen (or Fe/chalcogenide) layers [405, 406, 407, 408], respectively. Their quasi-two-dimensional crystal structures are also believed to play a significant role in the high $T_{c}$ values that are encountered in these families of compounds. In contrast, a quasi-two-dimensional, layered crystal structure is not observed universally among the superconducting heavy-fermion compounds; however, $\mathrm{CeCoIn}_{5}$ does serve as an interesting example wherein such a discussion is relevant. The cubic compound $\mathrm{CeIn}_{3}$ has an antiferromagnetic ground state that can be suppressed by applied pressure, leading to the emergence of superconductivity with $T_{c} \simeq 0.2 \mathrm{~K}$ [58]. This material is sometimes said to contain an infinite number of $\mathrm{CeIn}_{3}$ layers. The structurally-related compound, $\mathrm{CeCoIn}_{5}$, has a tetragonal crystal structure that is composed of alternating $\mathrm{CeIn}_{3}$ and $\mathrm{CoIn}_{2}$ layers. Instead of an antiferromagnetic ground state, $\mathrm{CeCoIn}_{5}$ exhibits superconductivity at ambient pressure with a $T_{c}$ that is an order of magnitude larger than in $\mathrm{CeIn}_{3}$ (i.e., $T_{c} \simeq 2.3 \mathrm{~K}$ ) [96]. In analogy with the cuprates and iron pnictide/chalcogenide compounds, its layered, quasi-twodimensional crystal structure is sometimes invoked to explain the large enhancement of $T_{c}$ in $\mathrm{CeCoIn}_{5}$ relative to that in CeIn 3 .
We have already discussed in the previous section that heavyfermion compounds are characterized by phase diagrams in which there is a close proximity (or sometimes coexistence) between superconductivity and a nearby magnetic or quadrupolar ordered state. This is also a common and defining element of the phase diagrams in superconducting cuprates and ironpnictide/chalcogenide compounds. As their antiferromagnetic or spin-density wave ordered states are suppressed by a nonthermal control parameter such as pressure or chemical composition, the magnetic phase boundaries tend to zero temperature, often terminating in a quantum critical point [409, 410, 411, 405, 406, 407, 408]; though, there are cases, such as in $\mathrm{LaFeAsO}_{1-x} \mathrm{~F}_{x}$, in which the ordered phase terminates in a firstorder phase transition [414] and there is no quantum critical point. When it is present, the quantum critical point is typically protected by a dome of superconductivity. In the phase space near the quantum critical point, the physical properties in the normal state are unconventional as manifested in the "strange metal" phase in the cuprates or non-Fermi liquid behavior in heavy-fermion compounds. At values of the control parameter greater than where the quantum critical point is located, there is usually a crossover to a Fermi liquid ground state. Generic examples of some of the phase diagrams that are encountered in these classes of materials are provided in Ref. [8].

There are also broad similarities between the superconducting energy gap symmetries of these unconventional classes of superconductors [204]. Superconductivity in the cuprates and most heavy-fermion compounds is believed to involve $d$ wave pairing. The energy gaps associated with such pairing are nodal and undergo sign changes between regions on the Fermi surface or surfaces separated by wave vector $\vec{Q}$ (i.e., $\operatorname{Sgn} \Delta(\vec{k}+\vec{Q})=-\operatorname{Sgn} \Delta(\vec{k}))$ where $\vec{Q}$ is associated with spin-fluctuations that are peaked at that momentum. The iron pnictide/chalcogenide compounds are generally thought to exhibit $s^{ \pm}$-pairing, which also involves a sign change of the gap; though, any nodes are typically accidental. The normally repulsive interactions involving spin fluctuations are rendered attractive when there is such a sign change in the energy gap, enabling unconventional quasiparticle pairing by spin fluctuations in these compounds [204]. When $\vec{k}=\vec{Q}$, a spin resonance feature is expected in inelastic neutron scattering experiments [204]. Such spin resonance features have been experimentally observed in the superconducting states of $\mathrm{CeCoIn}_{5}$ [200], $\mathrm{YBa}_{2} \mathrm{Cu}_{3} \mathrm{O}_{7}$ [415, 416, 417], $\mathrm{Bi}_{2} \mathrm{Sr}_{2} \mathrm{CaCu}_{2} \mathrm{O}_{8+\delta}$ [418], and $\mathrm{Ba}\left(\mathrm{Fe}_{0.925} \mathrm{Co}_{0.075}\right)_{2} \mathrm{As}_{2}$ [419]; these results in examples from each class of unconventional superconductor strongly support a common pairing mechanism via spin fluctuations.

We complete our discussion concerning the similarities between the classes of unconventional superconductors by considering an intriguing empirical observation plotted in Fig. 16. The $T_{c}$ values for various superconductors are plotted as a function of $T_{0}$, the frequency spread of the wave-vector-dependent part of the spin fluctuations (or a characteristic spin-fluctuation temperature), on a log-log plot. The line in Fig. 16 serves as a guide to the eye to emphasize an apparent relationship between 


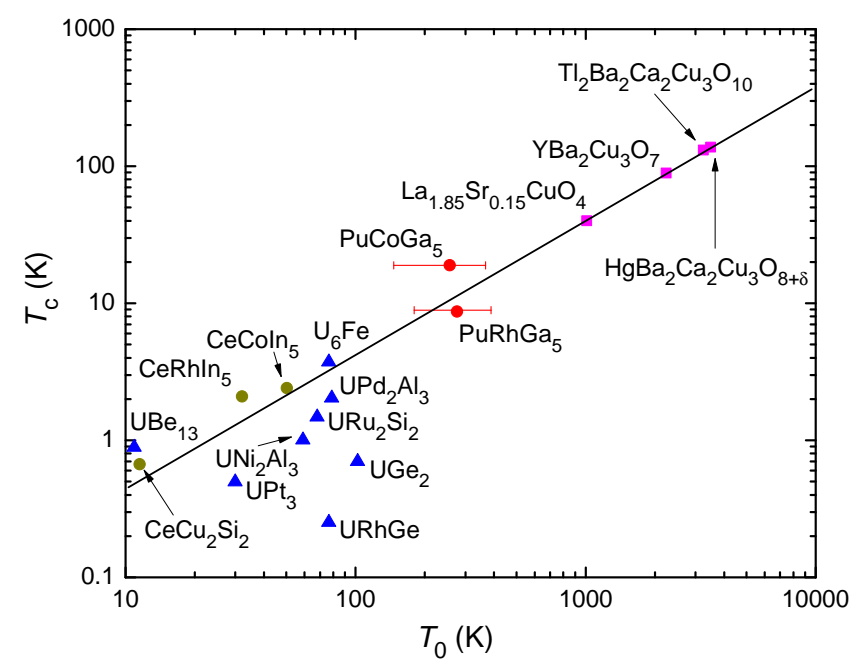

Figure 16: (Color online) Superconducting critical temperature $T_{c}$ plotted vs. characteristic spin fluctuation temperature $T_{0}$ on a log-log scale for several U-based compounds (blue triangles), Ce-based compounds (gold circles), $\mathrm{Pu}-$ based compounds (red circles), and cuprates (magenta squares). A roughly linear relationship between $T_{c}$ and $T_{0}$ is evident for the compounds in this plot, suggesting a relationship between spin fluctuations and superconductivity that is emphasized by a line that serves as a guide to the eye. Figure adapted from Refs. [420, 111].

$T_{c}$ and $T_{0}$. In practice, $T_{0}$ is obtained from an analysis of data from nuclear quadrupole resonance, specific heat, or electrical resistivity measurements $[420,421,111]$. We wish to emphasize two points concerning Fig. 16. First, there is a striking and continuous relationship between $T_{0}$ and $T_{c}$ among these compounds. Second, compounds like PuCoGa, $\mathrm{PuRhGa}_{5}$, and $\mathrm{U}_{6} \mathrm{Fe}$ fall between the heavy-fermion compounds and cuprates, bridging the void between them. Therefore, they share an intimate connection with the two classes of unconventional superconductors that bound them in Fig. 16. We might also briefly add that the effective quasiparticle mass $m^{*}$ that is often used to classify heavy-fermion compounds exists on a continuum. Furthermore, the hybridization between the localized $f$-and conduction-electron states, which is embodied in the FriedelAnderson model, includes both the Kondo limit (integral $f$ electron shell occupation number) and the valence-fluctuation limit (non-integral $f$-electron shell occupation number), both of which can lead to the heavy-fermion extreme. In this sense, the term "heavy-fermion physics" is probably a misnomer, since it represents one limit of a more general problem. In any case, this empirical relationship between $T_{c}$ and $T_{0}$ is consistent with a common pairing mechanism based on spin fluctuations for all of the compounds included in Fig. 16.

\subsection{Outlook for research on superconductivity in heavy- fermion compounds}

The current understanding of superconductivity in heavyfermion compounds is built upon 35 years of intense experimental and theoretical investigations. While many questions have been answered, there are numerous intriguing and unresolved issues to address, and more superconducting compounds to be discovered. In this selective review, we have attempted to provide a basic assessment of the current understanding of the normal- and superconducting-state properties of heavy-fermion compounds. We end by attempting to provide an outlook for the future of research in this fascinating and rich field of physics.

Until a comprehensive understanding of unconventional superconductivity is obtained, one obvious and vital way for the field to advance is to discover new heavy-fermion compounds with superconducting ground states, and to investigate their properties. There is a plot in Ref. [49] showing the number of known $f$-electron compounds with superconducting ground states (most of them are classified as heavy-fermion compounds in this article) as a function of year. From this plot, it appears that a new compound was discovered every two years (on average) between 1979 and 2000, after which, an average of nearly three new compounds were discovered per year up to 2009. This is strong evidence that components of a successful strategy for discovering new superconductors have already been identified and are being utilized. The future approach to discovering superconductivity in new heavy-fermion compounds should naturally build upon these components, which include studying strongly-correlated electron compounds based on $\mathrm{Ce}, \mathrm{Pr}, \mathrm{Yb}$, and $\mathrm{U}$, and subjecting such materials to measurements at lowtemperature under ambient and applied pressures. Measurements under applied pressure are particularly important when compounds exhibit magnetic (or electric quadrupole) ordered ground states that can be suppressed. New search strategies are also being identified; for example, the relatively recent discoveries of superconductivity in the heavy-fermion compounds $\operatorname{PrTi}_{2} \mathrm{Al}_{20}$ [31], $\operatorname{PrV}_{2} \mathrm{Al}_{20}$ [62], and $\beta$ - $\mathrm{YbAlB}_{4}$ [63] were only made possible by synthesizing and studying very high-quality single crystals. Superconductivity is not observed in samples of these compounds for which the measured residual resistivity ratio values fall below a sample-specific threshold. These examples demonstrate that exercising careful control of synthesis procedures to produce ultra-clean samples with low disorder may be extremely important when searching for superconductivity in some heavy-fermion compounds. It may also be rewarding to expand the search for superconductivity into relatively unexplored potential reservoirs of heavy-fermion compounds such as among the transition-metal oxides; though, we note that neither of the two known cases $\left(\mathrm{LiV}_{2} \mathrm{O}_{4}\right.$ [40] and $\mathrm{CaCu}_{3} \mathrm{Ru}_{4} \mathrm{O}_{12}$ [41]) exhibit superconductivity. The future of research on superconductivity in heavy-fermion compounds is full of promise, and there is every reason to believe that investigations of newly-discovered compounds as well as studies designed to obtain a more comprehensive understanding of known compounds will contribute to resolving the long-standing puzzle of how unconventional superconductivity works.

\section{Acknowledgements}

Work at the University of California, San Diego was performed with financial support from the U.S. Department of Energy, Office of Basic Energy Sciences, Division of Materials Sciences and Engineering under Award Grant No. DE-FG0204-ER46105, the National Science Foundation under Grant No. 
DMR-1206553, and the National Nuclear Security Administration under the Stewardship Science Academic Alliance program through the U.S. Department of Energy Grant No. DENA0001841. Work at Los Alamos was performed under the auspices of the U. S. Department of Energy and was supported by the U.S. DOE Office of Basic Energy Sciences, Division of Materials Sciences and Engineering.

[1] F. Steglich, J. Aarts, C. D. Bredl, W. Lieke, D. Meschede, W. Franz, H. Schäfer, Superconductivity in the presence of strong Pauli paramagnetism: $\mathrm{CeCu}_{2} \mathrm{Si}_{2}$, Phys. Rev. Lett. 43 (1979) 1892.

[2] L. N. Cooper, Bound electron pairs in a degenerate Fermi gas, Phys. Rev. 104 (1956) 1189.

[3] J. Bardeen, L. N. Cooper, J. R. Schrieffer, Microscopic theory of superconductivity, Phys. Rev. 106 (1957) 162.

[4] J. Bardeen, L. N. Cooper, J. R. Schrieffer, Theory of superconductivity, Phys. Rev. 108 (1957) 1175.

[5] L. D. Landau, Theory of Fermi-liquids, Sov. Phys. JETP 3 (1957) 920.

[6] L. D. Landau, Oscillations in a Fermi-liquid, Sov. Phys. JETP 5 (1957) 101.

[7] L. D. Landau, On the theory of the Fermi-liquid, Sov. Phys. JETP 8 (1959) 70.

[8] M. B. Maple, R. E. Baumbach, N. P. Butch, J. J. Hamlin, M. Janoschek, Non-Fermi liquid regimes and superconductivity in the low temperature phase diagrams of strongly correlated $d$ - and $f$-electron materials, J. Low Temp. Phys. 161 (2010) 4.

[9] K. Andres, J. E. Graebner, H. R. Ott, $4 f$-virtual-bound-state formation in $\mathrm{CeA}_{3}$ at low temperatures, Phys. Rev. Lett. 35 (1975) 1779.

[10] C. Kittel, Introduction to Solid State Physics, Eighth Edition, John Wiley \& Sons, 2005

[11] P. W. Anderson, Localized magnetic states in metals, Phys. Rev. 124 (1961) 41.

[12] W. J. de Haas, J. de Boer, G. J. van den Berg, The electrical resistance of gold, copper, and lead at low temperatures, Physica 1 (1933) 1115.

[13] J. Kondo, Resistance minimum in dilute magnetic alloys, Prog. Theor. Phys. 32 (1964) 37.

[14] J. R. Schrieffer, P. A. Wolff, Relation between the Anderson and Kondo Hamiltonians, Phys. Rev. 149 (1966) 491.

[15] H. v. Löhneysen, A. Rosch, M. Vojta, P. Wölfle, Fermi-liquid instabilities at magnetic quantum phase transitions, Rev. Mod. Phys. 79 (2007) 1015.

[16] P. Nozières, A "Fermi-liquid" description of the Kondo problem at low temperatures, J. Low Temp. Phys. 17 (1974) 31.

[17] K. G. Wilson, Renormalization group: Critical phenomena and Kondo problem, Rev. Mod. Phys. 47 (1975) 773.

[18] A. C. Hewson, The Kondo Problem to Heavy Fermions, Cambridge University Press, Cambridge, England, 1993.

[19] P. Coleman, Heavy fermions: electrons at the edge of magnetism, in: H. Krönmuller, S. Parkin (Eds.), Handbook of Magnetism and Advanced Magnetic Materials, Vol. 1, John Wiley and Sons, Ltd., 2007.

[20] K. Kadowaki, S. B. Woods, Universal relationship of the resistivity and specific heat in heavy-fermion compounds, Solid State Comm. 58 (1986) 507.

[21] N. Tsujii, H. Kontani, K. Yoshimura, Universality in heavy fermion systems with general degeneracy, Phys. Rev. Lett. 94 (2005) 057201.

[22] T. Pruschke, R. Bulla, M. Jarrell, Low-energy scale of the periodic Anderson model, Phys. Rev. B 61 (2000) 12799.

[23] S. Nakatsuji, S. Yeo, L. Balicas, Z. Fisk, P. Schlottmann, P. G. Pagliuso, N. O. Moreno, J. L. Sarrao, J. D. Thompson, Intersite coupling effects in a Kondo lattice, Phys. Rev. Lett. 89 (2002) 106402.

[24] S. Nakatsuji, D. Pines, Z. Fisk, Two fluid description of the Kondo lattice, Phys. Rev. Lett. 92 (2004) 016401.

[25] Y.-F. Yang, Z. Fisk, H.-O. Lee, J. D. Thompson, D. Pines, Scaling the Kondo lattice, Nature 454 (2008) 611.

[26] C. M. Varma, Mixed-valence compounds, Rev. Mod. Phys. 48 (1976) 219.

[27] D. L. Cox, Quadrupolar Kondo effect in uranium heavy-electron materials?, Phys. Rev. Lett. 59 (1987) 1240.

[28] D. L. Cox, M. Makivic, Phenomenology of two channel Kondo alloys and compounds, Physica B 199 \& 200 (1994) 391.
[29] D. L. Cox, A. Zawadowski, Exotic Kondo effects in metals: magnetic ions in a crystalline electric field and tunnelling centres, Adv. Phys. 47 (1998) 599.

[30] A. Yatskar, W. P. Beyermann, R. Movshovich, P. C. Canfield, Possible correlated-electron behavior from quadrupolar fluctuations in $\operatorname{PrInAg} \mathrm{A}_{2}$, Phys. Rev. Lett. 77 (1996) 3637.

[31] K. Matsubayashi, T. Tanaka, A. Sakai, S. Nakatsuji, Y. Kubo, Y. Uwatoko, Pressure-induced heavy fermion superconductivity in the nonmagnetic quadrupolar system $\operatorname{PrTi}_{2} \mathrm{Al}_{20}$, Phys. Rev. Lett. 109 (2012) 187004.

[32] E. D. Bauer, N. A. Frederick, P.-C. Ho, V. S. Zapf, M. B. Maple, Superconductivity and heavy fermion behavior in $\mathrm{PrOs}_{4} \mathrm{Sb}_{12}$, Phys. Rev. B 65 (2002) 100506(R).

[33] Y. Aoki, T. Tayama, T. Sakakibara, K. Kuwahara, K. Isawa, M. Kohgi, W. Higemoto, D. E. MacLaughlin, H. Sugawara, H. Sato, The unconventional superconductivity of Skutterudite $\mathrm{PrOs}_{4} \mathrm{Sb}_{12}$ : Time-reversal symmetry breaking and adjacent field-induced quadrupole ordering, J. Phys. Soc. Jpn. 76 (2007) 051006.

[34] J. Jensen, A. R. Mackintosh, Rare Earth Magnetism: Structures and Excitations, International Series of Monographs on Physics, Clarendon Press, Oxford, 1991.

[35] M. A. Ruderman, C. Kittel, Indirect exchange coupling of nuclear magnetic moments by conduction electrons, Phys. Rev. 96 (1954) 99.

[36] T. Kasuya, A theory of metallic ferro- and antiferromagnetism on Zener's model, Prog. Theor. Phys. 16 (1956) 45.

[37] K. Yosida, Magnetic properties of Cu-Mn alloys, Phys. Rev. 106 (1957) 893.

[38] S. Doniach, The Kondo lattice and weak antiferromagnetism, Physica B \& C 91 (1977) 231.

[39] P. Gegenwart, Q. Si, F. Steglich, Quantum criticality in heavy-fermion metals, Nat. Phys. 4 (2008) 186.

[40] S. Kondo, D. C. Johnston, C. A. Swenson, F. Borsa, A. V. Mahajan, L. L. Miller, T. Gu, A. I. Goldman, M. B. Maple, D. A. Gajewski, E. J. Freeman, N. R. Dilley, R. P. Dickey, J. Merrin, K. Kojima, G. M. Luke, Y. J. Uemura, O. Chmaissem, J. D. Jorgensen, $\mathrm{LiV}_{2} \mathrm{O}_{4}$ : A heavy fermion transition metal oxide, Phys. Rev. Lett. 78 (1997) 3729.

[41] W. Kobayashi, I. Terasaki, J. Takeya, I. Tskukada, Y. Ando, A novel heavy-fermion state in $\mathrm{CaCu}_{3} \mathrm{Ru}_{4} \mathrm{O}_{12}$, J. Phys. Soc. Jpn. 73 (2004) 2373.

[42] G. R. Stewart, Heavy-fermion systems, Rev. Mod. Phys. 56 (1984) 755.

[43] H. R. Ott, in: Progress in Low Temperature Physics, Vol. XI, Elsevier, 1987.

[44] N. Grewe, F. Steglich, Heavy fermions, in: K. A. Gschneidner Jr., L. Eyring (Eds.), Handbook on the Physics and Chemistry of Rare Earths, Vol. 14, Elsevier, 1991, Ch. 97.

[45] M. B. Maple, Three decades of prospecting for novel electronic states and phenomena in $f$-electron materials, J. Alloys and Comp. 303-304 (2000) 1 .

[46] M. B. Maple, Strongly correlated electron phenomena in f-electron materials, J. Phys. Soc. Jpn. 74 (2005) 222.

[47] J. Flouquet, On the heavy fermion road, in: W. P. Halperin (Ed.), Progress in Low Temperature Physics, Vol. XV, Elsevier, 2005, Ch. 2.

[48] P. S. Riseborough, G. M. Schmiedeshoff, J. L. Smith, Heavy-fermion superconductivity, in: K.-H. Bennemann, J. B. Ketterson (Eds.), Superconductivity: Novel Superconductors, Vol. 2, Springer-Verlag, 2008, Ch. 19.

[49] C. Pfleiderer, Superconducting phases of $f$-electron compounds, Rev. Mod. Phys. 81 (2009) 1551.

[50] E. Bucher, J. P. Maita, G. W. Hull, R. C. Fulton, A. S. Cooper, Electronic properties of beryllides of the rare earth and some actinides, Phys. Rev. B 11 (1975) 440.

[51] D. D. Osheroff, R. C. Richardson, D. M. Lee, Evidence for a new phase of solid $\mathrm{He}^{3}$, Phys. Rev. Lett. 28 (1972) 885.

[52] H. R. Ott, H. Rudigier, Z. Fisk, J. L. Smith, $\mathrm{UBe}_{13}$ : An unconventional actinide superconductor, Phys. Rev. Lett. 50 (1983) 1595.

[53] G. R. Stewart, Z. Fisk, J. O. Willis, J. L. Smith, Possibility of coexistence of bulk superconductivity and spin fluctuations in $\mathrm{UPt}_{3}$, Phys. Rev. Lett. 52 (1984) 679

[54] T. T. M. Palstra, A. A. Menovsky, J. van den Berg, A. J. Dirkmaat, P. H. Kes, G. J. Nieuwenhuys, J. A. Mydosh, Superconducting and magnetic transitions in the heavy-fermion system $\mathrm{URu}_{2} \mathrm{Si}_{2}$, Phys. Rev. Lett. 55 (1985) 2727. 
[55] M. B. Maple, J. W. Chen, Y. Dalichaouch, T. Kohara, C. Rossel, M. S Torikachvili, M. W. McElfresh, J. D. Thompson, Partially gapped Fermi surface in the heavy-electron superconductor $\mathrm{URu}_{2} \mathrm{Si}_{2}$, Phys. Rev. Lett. 56 (1986) 185

[56] W. Schlabitz, J. Baumann, B. Pollit, U. Rauchschwalbe, H. M. Mayer, U. Ahlheim, C. D. Bredl, Superconductivity and magnetic order in a strongly interacting Fermi-system: $\mathrm{URu}_{2} \mathrm{Si}_{2}$, Z. Phys. B 62 (1986) 171.

[57] F. M. Grosche, S. R. Julian, N. D. Mathur, G. G. Lonzarich, Magnetic and superconducting phases of $\mathrm{CePd}_{2} \mathrm{Si}_{2}$, Physica B 223 \& 224 (1996) 50.

[58] N. D. Mathur, F. M. Grosche, S. R. Julian, I. R. Walker, D. M. Freye, R. K. W. Haselwimmer, G. G. Lonzarich, Magnetically mediated superconductivity in heavy fermion compounds, Nature 394 (1998) 39

[59] N. R. Werthamer, E. Helfand, P. C. Hohenberg, Temperature and purity dependence of the superconducting critical field, $H_{c 2}$. III. electron spin and spin-orbit effects, Phys. Rev. 147 (1966) 295.

[60] M. Sigrist, K. Ueda, Phenomenological theory of unconventional superconductivity, Rev. Mod. Phys. 63 (1991) 239.

[61] M. B. Maple, E. D. Bauer, V. S. Zapf, J. Wosnitza, Unconventional superconductivity in novel materials, in: K. H. Bennemann, J. B. Ketterson (Eds.), The Physics of Superconductors, Vol. II, Springer-Verlag, 2004, Ch. 8.

[62] M. Tsujimoto, Y. Matsumoto, T. Tomita, A. Sakai, S. Nakatsuji, Heavyfermion superconductivity in the quadrupole ordered state of $\operatorname{PrV}_{2} \mathrm{Al}_{20}$, Phys. Rev. Lett. 113 (2014) 267001.

[63] S. Nakatsuji, K. Kuga, Y. Machida, T. Tayama, T. Sakakibara, Y. Karaki, H. Ishimoto, S. Yonezawa, Y. Maeno, E. Pearson, G. G. Lonzarich, L. Balicas, H. Lee, Z. Fisk, Superconductivity and quantum criticality in the heavy-fermion system $\beta$-YbAlB 4 , Nature Phys. 4 (2008) 603.

[64] D. Jaccard, K. Behnia, J. Sierro, Pressure induced heavy fermion superconductivity of $\mathrm{CeCu}_{2} \mathrm{Ge}_{2}$, Phys. Lett. A 163 (1992) 475.

[65] R. Movshovich, T. Graf, D. Mandrus, J. D. Thompson, J. L. Smith, Z. Fisk, Superconductivity in heavy-fermion $\mathrm{CeRh}_{2} \mathrm{Si}_{2}$, Phys. Rev. B 53 (1996) 8241

[66] W. Assmus, M. Herrmann, U. Rauchschwalbe, S. Riegel, W. Lieke, H. Spille, S. Horn, G. Weber, F. Steglich, G. Cordier, Superconductivity in $\mathrm{CeCu}_{2} \mathrm{Si}_{2}$ single crystals, Phys. Rev. Lett. 52 (1984) 469.

[67] O. Trovarelli, M. Weiden, R. Müller-Reisener, M. Gómez-Berisso, P. Gegenwart, M. Deppe, C. Geibel, J. G. Sereni, F. Steglich, Evolution of magnetism and superconductivity in $\mathrm{CeCu}_{2}\left(\mathrm{Si}_{1-x} \mathrm{Ge}_{x}\right)_{2}$, Phys. Rev. B 56 (1997) 678.

[68] F. G. Aliev, N. B. Brandt, V. V. Moshchalkov, S. M. Chudinov, Superconductivity in $\mathrm{CeCu}_{2} \mathrm{Si}_{2}$, Solid State Comm. 45 (1983) 215.

[69] F. M. Grosche, P. Agarwal, S. R. Julian, N. J. Wilson, R. K. W. Haselwimmer, S. J. S. Lister, N. D. Mathur, F. V. Carter, S. S. Saxena, G. G. Lonzarich, Anomalous low temperature states in $\mathrm{CeNi}_{2} \mathrm{Ge}_{2}$ and $\mathrm{CePd}_{2} \mathrm{Si}_{2}$, J. Phys.: Condens. Matter 12 (2000) L533.

[70] O. Stockert, E. Faulhaber, G. Zwicknagl, N. Stüßer, H. S. Jeevan, M. Deppe, R. Borth, R. Küchler, M. Loewenhaupt, C. Geibel, F. Steglich, Nature of the A phase in $\mathrm{CeCu}_{2} \mathrm{Si}_{2}$, Phys. Rev. Lett. 92 (2004) 136401.

[71] A. M. Clogston, Upper limit for the critical field in hard superconductors, Phys. Rev. Lett. 9 (1962) 266.

[72] U. Rauchschwalbe, W. Lieke, C. D. Bredl, F. Steglich, J. Aarts, K. M. Martini, A. C. Mota, Critical fields of the "heavy-fermion" superconductor $\mathrm{CeCu}_{2} \mathrm{Si}_{2}$, Phys. Rev. Lett. 49 (1982) 1448.

[73] M. Lang, R. Modler, U. Ahlheim, R. Helfrich, P. H. P. Reinders, F. Steglich, W. Assmus, W. Sun, G. Bruls, D. Weber, B. Lüthi, Cooperative effects in $\mathrm{CeCu}_{2} \mathrm{Si}_{2}$, Phys. Scripta T39 (1991) 135.

[74] Y. Kawasaki, K. Ishida, S. Kawasaki, T. Mito, G. Zheng, Y. Kitaoka, C. Geibel, F. Steglich, Exotic superconductivity in the coexistent phase of antiferromagnetism and superconductivity in $\mathrm{CeCu}_{2}\left(\mathrm{Si}_{0.98} \mathrm{Ge}_{0.02}\right)_{2}$ : A Cu-NQR study under hydrostatic pressure, J. Phys. Soc. Jpn. 73 (2004) 194.

[75] S. Kittaka, Y. Aoki, Y. Shimura, T. Sakakibara, S. Seiro, C. Geibel, F. Steglich, H. Ikeda, K. Machida, Multiband superconductivity with unexpected deficiency of nodal quasiparticles in $\mathrm{CeCu}_{2} \mathrm{Si}_{2}$, Phys. Rev. Lett. 112 (2014) 067002.

[76] B. Bellarbi, A. Benoit, D. Jaccard, J. M. Mignot, H. F. Braun, High-pressure valence instability and $T_{c}$ maximum in superconducting $\mathrm{CeCu}_{2} \mathrm{Si}_{2}$, Phys. Rev. B 30 (1984) 1182.
[77] H. Q. Yuan, F. M. Grosche, M. Deppe, C. Geibel, G. Sparn, F. Steglich, Observation of two distinct superconducting phases in $\mathrm{CeCu}_{2} \mathrm{Si}_{2}$, Science 302 (2003) 2104

[78] A. T. Holmes, D. Jaccard, K. Miyake, Signatures of valence fluctuations in $\mathrm{CeCu}_{2} \mathrm{Si}_{2}$ under high pressure, Phys. Rev. B 69 (2004) 024508.

[79] G. Knopp, A. Loidl, K. Knorr, L. Pawlak, M. Duczmal, R. Caspary, U. Gottwick, H. Spille, F. Steglich, A. P. Murani, Magnetic order in a Kondo lattice: a neutron scattering study of $\mathrm{CeCu}_{2} \mathrm{Ge}_{2}$, Z. Phys. B 77 (1989) 95 .

[80] R. A. Fisher, J. P. Emerson, R. Caspary, N. E. Phillips, F. Steglich, The low-temperature specific heat of $\mathrm{CeCu}_{2} \mathrm{Ge}_{2}$ at 0 and $9.5 \mathrm{kbar}$, Physica $\mathrm{B}$ 194-196 (1994) 459

[81] B. H. Grier, J. M. Lawrence, V. Murgai, R. D. Parks, Magnetic ordering in $\mathrm{Ce}_{2} \mathrm{Si}_{2}(M=\mathrm{Ag}, \mathrm{Au}, \mathrm{Pd}, \mathrm{Rh})$ compounds as studied by neutron diffraction, Phys. Rev. B 29 (1984) 2664

[82] J. D. Thompson, R. D. Parks, H. Borges, Effect of pressure on the Néel temperature of Kondo-lattice systems, J. Mag. Mag. Mat. 54-57 (1986) 377.

[83] I. Sheikin, E. Steep, D. Braithwaite, J.-P. Brison, S. Raymond, D. Jaccard, J. Flouquet, Superconductivity, upper critical field and anomalous normal state in $\mathrm{CePd}_{2} \mathrm{Si}_{2}$ near the quantum critical point, J. Low Temp. Phys. 122 (2001) 591.

[84] R. A. Steeman, E. Frikkee, R. B. Helmholdt, A. A. Menovsky, J. van den Berg, G. J. Nieuwenhuys, J. A. Mydosh, $\mathrm{CePd}_{2} \mathrm{Si}_{2}$ : A reduced-moment antiferromagnet, Solid State Comm. 66 (1988) 103.

[85] S. Kawarazaki, M. Sato, Y. Miyako, N. Chigusa, K. Watanabe, N. Metoki, Y. Koike, M. Nishi, Ground-state magnetic structure of $\mathrm{CeRh}_{2} \mathrm{Si}_{2}$ and the response to hydrostatic pressure as studied by neutron diffraction, Phys. Rev. B 61 (2000) 4167.

[86] T. Graf, J. D. Thompson, M. F. Hundley, R. Movshovich, Z. Fisk, D. Mandrus, R. A. Fisher, N. E. Phillips, Comparison of $\mathrm{CeRh}_{2} \mathrm{Si}_{2}$ and $\mathrm{CeRh}_{2-x} \mathrm{Ru}_{x} \mathrm{Si}_{2}$ near their magnetic-nonmagnetic boundaries, Phys. Rev. Lett. 78 (1997) 3769.

[87] A. Villaume, D. Aoki, Y. Haga, G. Knebel, R. Boursier, J. Flouquet, Collapse of antiferromagnetism in $\mathrm{CeRh}_{2} \mathrm{Si}_{2}$ : volume versus entropy, $\mathrm{J}$. Phys.: Condens. Matter 20 (2008) 015203.

[88] R. Settai, T. Takeuchi, Y. Ōnuki, Recent advances in Ce-based heavyfermion superconductivity and Fermi surface properties, J. Phys. Soc. Jpn. 76 (2007) 051003

[89] B. Fåk, J. Flouquet, G. Lapertot, T. Fukuhara, H. Kadowaki, Magnetic correlations in single-crystalline $\mathrm{CeNi}_{2} \mathrm{Ge}_{2}$, J. Phys.:Condens. Matter 12 (2000) 5423.

[90] G. Knopp, A. Loidl, R. Caspary, U. Gottwick, C. D. Bredl, H. Spille, F. Steglich, A. P. Murani, Specific heat, resistivity and neutron scattering studies in the Kondo lattice $\mathrm{CeNi}_{2} \mathrm{Ge}_{2}$, J. Mag. Mag. Mater. 74 (1988) 341.

[91] P. Gegenwart, F. Kromer, M. Lang, G. Sparn, C. Geibel, F. Steglich, Non-Fermi-liquid effects at ambient pressure in a stoichiometric heavyfermion compound with very low disorder: $\mathrm{CeNi}_{2} \mathrm{Ge}_{2}$, Phys. Rev. Lett. 82 (1999) 1293.

[92] S. J. S. Lister, F. M. Grosche, F. V. Carter, R. K. W. Haselwimmer, S. S. Saxena, N. D. Mathur, S. R. Julian, G. G. Lonzarich, Quantum critical behaviour in the $\mathrm{CePd}_{2} \mathrm{Si}_{2} / \mathrm{CeNi}_{2} \mathrm{Ge}_{2}$ system, Z. Phys. B 103 (1997) 263.

[93] T. Park, F. Ronning, H. Q. Yuan, M. B. Salamon, R. Movshovich, J. L. Sarrao, J. D. Thompson, Hidden magnetism and quantum criticality in the heavy fermion superconductor CeRhIn ${ }_{5}$, Nature 440 (2006) 65.

[94] H. Hegger, C. Petrovic, E. G. Moshopoulou, M. F. Hundley, J. L. Sarrao, Z. Fisk, J. D. Thompson, Pressure-induced superconductivity in quasi2D CeRhIn 5 , Phys. Rev. Lett. 84 (2000) 4986.

[95] P. Monthoux, G. G. Lonzarich, Magnetically mediated superconductivity: Crossover from cubic to tetragonal lattice, Phys. Rev. B 66 (2002) 224504

[96] C. Petrovic, P. G. Pagliuso, M. F. Hundley, R. Movshovich, J. L. Sarrao, J. D. Thompson, Z. Fisk, P. Monthoux, Heavy-fermion superconductivity in CeCoIn 5 at 2.3 K, J. Phys.: Condens. Matter 13 (2001) L337.

[97] C. Petrovic, R. Movshovich, M. Jaime, P. G. Pagliuso, M. F. Hundley, J. L. Sarrao, Z. Fisk, J. D. Thompson, A new heavy-fermion superconductor CeIrIn 5 : A relative of the cuprates?, Europhys. Lett. 53 (2001) 354.

[98] Y. N. Grin, Y. P. Yarmolyuk, E. I. Giadyshevskii, Crystal structures of 
$\mathrm{R}_{2} \mathrm{CoGa}_{8}$ compounds $(\mathrm{R}=\mathrm{Sm}, \mathrm{Gd}, \mathrm{Tb}, \mathrm{Dy}, \mathrm{Ho}, \mathrm{Er}, \mathrm{Tm}, \mathrm{Lu}, \mathrm{Y})$ and $\mathrm{RCoGa}_{5}$ compounds $(\mathrm{R}=\mathrm{Gd}, \mathrm{Tb}, \mathrm{Dy}, \mathrm{Ho}, \mathrm{Er}, \mathrm{Tm}, \mathrm{Lu}, \mathrm{Y})$, Sov. Phys. Crystallogr. 24 (1979) 137.

[99] V. A. Sidorov, X. Lu, T. Park, H. Lee, P. H. Tobash, R. E. Baumbach, F. Ronning, E. D. Bauer, J. D. Thompson, Pressure phase diagram and quantum criticality of $\mathrm{CePt}_{2} \mathrm{In}_{7}$ single crystals, Phys. Rev. B 88 (2013) 020503(R).

[100] G. Chen, S. Ohara, M. Hedo, Y. Uwatoko, K. Saito, M. Sorai, I. Sakamoto, Observation of superconductivity in heavy-fermion compounds of $\mathrm{Ce}_{2} \mathrm{CoIn}_{8}$, J. Phys. Soc. Jpn. 71 (2002) 2836.

[101] A. L. Cornelius, P. G. Pagliuso, M. F. Hundley, J. L. Sarrao, Fieldinduced magnetic transitions in the quasi-two-dimensional heavyfermion antiferromagnets $\mathrm{Ce}_{n} \mathrm{RhIn}_{3 n+2}(n=1$ or 2), Phys. Rev. B 64 (2001) 144411.

[102] M. Nicklas, V. A. Sidorov, H. A. Borges, P. G. Pagliuso, C. Petrovic, Z. Fisk, J. L. Sarrao, J. D. Thompson, Magnetism and superconductivity in $\mathrm{Ce}_{2} \mathrm{RhIn}_{8}$, Phys. Rev. B 67 (2003) 020506(R).

[103] D. Kaczorowski, D. Gnida, A. P. Pikul, V. H. Tran, Heavy-fermion superconductivity in $\mathrm{Ce}_{2} \mathrm{PdIn}_{8}$, Solid State Comm. 150 (2010) 411.

[104] M. Kratochvílová, J. Prokleška, K. Uhlírová, M. Dušek, V. Sechovsky, J. Custers, Ambient pressure superconductivity emerging in the local moment antiferromagnetic phase of $\mathrm{Ce}_{3} \mathrm{PdIn}_{11}$, arXiv:1403.7010v2 [cond-mat.str-el].

[105] E. D. Bauer, M. M. Altarawneh, P. H. Tobash, K. Gofryk, O. E. AyalaValenzuela, J. N. Mitchell, R. D. McDonald, C. H. Mielke, F. Ronning, J.-C. Griveau, E. Colineau, R. Eloirdi, R. Caciuffo, B. L. Scott, O. Janka, S. M. Kauzlarich, J. D. Thompson, Localized $5 f$ electrons in superconducting PuCoIn 5 : consequences for superconductivity in $\mathrm{PuCoGa}_{5}, \mathrm{~J}$. Phys.: Condens. Matter 24 (2012) 052206.

[106] E. D. Bauer, unpublished (2014).

[107] J. L. Sarrao, L. A. Morales, J. D. Thompson, B. L. Scott, G. R. Stewart, F. Wastin, J. Rebizant, P. Boulet, E. Colineau, G. H. Lander, Plutoniumbased superconductivity with a transition temperature above $18 \mathrm{~K}$, Nature 420 (2002) 297.

[108] F. Wastin, P. Boulet, J. Rebizant, E. Colineau, G. H. Lander, Advances in the preparation and characterization of transuranium systems, J. Phys.: Condens. Matter 15 (2003) S2279.

[109] H. Shishido, R. Settai, D. Aoki, S. Ikeda, H. Nakawaki, N. Nakamura, T. Iizuka, Y. Inada, K. Sugiyama, T. Takeuchi, K. Kindo, T. C. Kobayashi, Y. Haga, H. Harima, Y. Aoki, T. Namiki, H. Sato, Y. Ōnuki, Fermi surface, magnetic and superconducting properties of $\mathrm{LaRhIn}_{5}$ and $\mathrm{CeTIn}_{5}$ (T: Co, Rh and Ir), J. Phys. Soc. Jpn. 71 (2002) 162.

[110] N. Harrison, U. Alver, R. G. Goodrich, I. Vekhter, J. L. Sarrao, P. G. Pagliuso, N. O. Moreno, L. Balicas, Z. Fisk, D. Hall, R. T. Macaluso, J. Y. Chan, $4 f$-electron localization in $\mathrm{Ce}_{x} \mathrm{La}_{1-x} M \mathrm{In}_{5}$ with $M=\mathrm{Co}, \mathrm{Rh}$, or Ir, Phys. Rev. Lett. 93 (2004) 186405.

[111] J. L. Sarrao, J. D. Thompson, Superconductivity in cerium- and plutonium-based '115' materials, J. Phys. Soc. Jpn. 76 (2007) 051013.

[112] K. Izawa, H. Yamaguchi, Y. Matsuda, H. Shishido, R. Settai, Y. Onuki, Angular position of nodes in the superconducting gap of quasi-2D heavy-fermion superconductor $\mathrm{CeCoIn}_{5}$, Phys. Rev. Lett. 87 (2001) 057002.

[113] K. An, T. Sakakibara, R. Settai, Y. Onuki, M. Hiragi, M. Ichioka, K. Machida, Sign reversal of field-angle resolved heat capacity oscillations in a heavy fermion superconductor CeCoIn 5 and $d_{x^{2}-y^{2}}$ pairing symmetry, Phys. Rev. Lett. 104 (2010) 037002.

[114] L. H. Greene, W. K. Park, J. L. Sarrao, J. D. Thompson, Pointcontact spectroscopy of $\mathrm{CeCoIn}_{5}$ : Andreev reflection studies of the normal-metal-heavy-fermion superconductor interface, Physica B 378380 (2006) 671

[115] B. B. Zhou, S. Misra, E. H. da Silva Neto, P. Aynajian, R. E. Baumbach, J. D. Thompson, E. D. Bauer, A. Yazdani, Visualizing nodal heavy fermion superconductivity in CeCoIn 5 , Nature Phys. 9 (2013) 474.

[116] M. P. Allan, F. Massee, D. K. Morr, J. V. Dyke, A. Rost, A. P. Mackenzie, C. Petrovic, J. C. Davis, Imaging Cooper pairing of heavy fermions in $\mathrm{CeCoIn}_{5}$, Nature Phys. 9 (2013) 468.

[117] D. A. Wollman, D. J. Van Harlingen, W. C. Lee, D. M. Ginsberg, A. J. Leggett, Experimental determination of the superconducting pairing state in YBCO from the phase coherence of YBCO-Pb dc SQUIDs, Phys. Rev. Lett. 71 (1993) 2134.

[118] T. Timusk, B. Statt, The pseudogap in high-temperature superconduc- tors: an experimental survey, Rep. Prog. Phys. 62 (1999) 61.

[119] V. A. Sidorov, M. Nicklas, P. G. Pagliuso, J. L. Sarrao, Y. Bang, A. V. Balatsky, J. D. Thompson, Superconductivity and quantum criticality in CeCoIn 5 , Phys. Rev. Lett. 89 (2002) 157004.

[120] S. Wirth, Y. Prots, M. Wedel, S. Ernst, S. Kirchner, Z. Fisk, J. D. Thompson, F. Steglich, Y. Grin, Structural investigations of CeIrIn 5 and $\mathrm{CeCoIn}_{5}$ on macroscopic and atomic length scales, J. Phys. Soc. Jpn. 83 (2014) 061009.

[121] R. Movshovich, M. Jaime, J. D. Thompson, C. Petrovic, Z. Fisk, P. G. Pagliuso, J. L. Sarrao, Unconventional superconductivity in CeIrIn 5 and CeCoIn 5 : Specific heat and thermal conductivity studies, Phys. Rev. Lett. 86 (2001) 5152.

[122] M. A. Tanatar, J. Paglione, S. Nakatsuji, D. G. Hawthorn, E. Boaknin, R. W. Hill, F. Ronning, M. Sutherland, L. Taillefer, C. Petrovic, P. C. Canfield, Z. Fisk, Unpaired electrons in the heavy-fermion superconductor CeCoIn 5 , Phys. Rev. Lett. 95 (2005) 067002.

[123] Y. Kohori, Y. Yamato, Y. Iwamoto, T. Kohara, E. D. Bauer, M. B. Maple, J. L. Sarrao, NMR and NQR studies of the heavy fermion superconductors $\mathrm{Ce} T \operatorname{In}_{5}(T=$ Co and Ir), Phys. Rev. B 64 (2001) 134526.

[124] J. D. Thompson, M. Nicklas, A. Bianchi, R. Movshovich, A. Llobet, W. Bao, A. Malinowski, M. F. Hundley, N. O. Moreno, P. G. Pagliuso, J. L. Sarrao, S. Nakatsuji, Z. Fisk, R. Borth, E. Lengyel, N. Oeschler, G. Sparn, F. Steglich, Magnetism and unconventional superconductivity in $\mathrm{Ce}_{n} \mathrm{M}_{m} \mathrm{In}_{3 n+2 m}$ heavy-fermion crystals, Physica B 329-333 (2003) 446.

[125] X. Lu, H. Lee, T. Park, F. Ronning, E. D. Bauer, J. D. Thompson, Heatcapacity measurements of energy-gap nodes of the heavy-fermion superconductor CeIrIn ${ }_{5}$ deep inside the pressure-dependent dome structure of its superconducting phase diagram, Phys. Rev. Lett. 108 (2012) 027001.

[126] S. Kittaka, Y. Aoki, T. Sakakibara, A. Sakai, S. Nakatsuji, Y. Tsutsumi, M. Ichioka, K. Machida, Superconducting gap structure of CeIrIn from field-angle-resolved measurements of its specific heat, Phys. Rev. B 85 (2012) 060505(R).

[127] T. Park, E. D. Bauer, J. D. Thompson, Probing the nodal gap in the pressure-induced heavy fermion superconductor CeRhIn ${ }_{5}$, Phys. Rev. Lett. 101 (2008) 177002.

[128] M. Hedo, N. Kurita, Y. Uwatoko, G. Chen, S. Ohara, I. Sakamoto, Superconducting properties of new heavy fermion superconductor $\mathrm{Ce}_{2} \mathrm{CoIn}_{8}$, J. Mag. Mag. Mater. 272-276 (2004) 146.

[129] S. Nair, S. Wirth, M. Nicklas, J. L. Sarrao, J. D. Thompson, Z. Fisk, F. Steglich, Precursor state to unconventional superconductivity in CeIrIn 5 , Phys. Rev. Lett. 100 (2008) 137003.

[130] J. D. Thompson, Z. Fisk, Progress in heavy-fermion superconductivity: Ce115 and related materials, J. Phys. Soc. Jpn. 81 (2012) 011002.

[131] T. Park, H. Lee, I. Martin, X. Lu, V. A. Sidorov, K. Gofryk, F. Ronning, E. D. Bauer, J. D. Thompson, Textured superconducting phase in the heavy fermion CeRhIn 5 , Phys. Rev. Lett. 108 (2012) 077003.

[132] E. Lengyel, J. L. Sarrao, G. Sparn, F. Steglich, J. D. Thompson, Heat capacity of $\mathrm{Ce}_{2} \mathrm{RhIn}_{8}$ under hydrostatic pressure, J. Mag. Mag. Mater. 272-276 (2004) 52.

[133] S. Ikeda, H. Shishido, M. Nakashima, R. Settai, D. Aoki, Y. Haga, H. Harima, Y. Aoki, T. Namiki, H. Sato, Y. Ōnuki, Unconventional superconductivity in $\mathrm{CeCoIn}_{5}$ studied by the specific heat and magnetization measurements, J. Phys. Soc. Jpn. 70 (2001) 2248.

[134] T. P. Murphy, D. Hall, E. C. Palm, S. W. Tozer, C. Petrovic, Z. Fisk, R. G. Goodrich, P. G. Pagliuso, J. L. Sarrao, J. D. Thompson, Anomalous superconductivity and field-induced magnetism in $\mathrm{CeCoIn}_{5}$, Phys. Rev. B 65 (2002) 100514(R).

[135] E. E. M. Chia, D. J. Van Harlingen, M. B. Salamon, B. D. Yanoff, I. Bonalde, J. L. Sarrao, Nonlocality and strong coupling in the heavy fermion superconductor $\mathrm{CeCoIn}_{5}$ : A penetration depth study, Phys. Rev. B 67 (2003) 014527.

[136] W. K. Park, J. L. Sarrao, J. D. Thompson, L. H. Greene, Andreev reflection in heavy-fermion superconductors and order parameter symmetry in CeCoIn 5 , Phys. Rev. Lett. 100 (2008) 177001.

[137] R. J. Ormeno, A. Sibley, C. E. Gough, S. Sebastian, I. R. Fisher, Microwave conductivity and penetration depth in the heavy fermion superconductor $\mathrm{CeCoIn}_{5}$, Phys. Rev. Lett. 88 (2002) 047005.

[138] S. Özcan, D. M. Broun, B. Morgan, R. K. W. Haselwimmer, J. L. Sarrao, S. Kamal, C. P. Bidinosti, P. J. Turner, M. Raudsepp, J. R. Waldram, London penetration depth measurements of the heavy-fermion super- 
conductor $\mathrm{CeCoIn}_{5}$ near a magnetic quantum critical point, Europhys. Lett. 62 (2003) 412 .

[139] T. Park, J. D. Thompson, Magnetism and superconductivity in strongly correlated CeRhIn 5 , New J. Phys. 11 (2009) 055062.

[140] T. Mito, S. Kawasaki, G. q. Zheng, Y. Kawasaki, K. Ishida, Y. Kitaoka, D. Aoki, Y. Haga, Y. Onuki, Pressure-induced anomalous magnetism and unconventional superconductivity in $\mathrm{CeRhIn}_{5}:{ }^{115} \mathrm{In}-\mathrm{NQR}$ study under pressure, Phys. Rev. B 63 (2001) 220507(R).

[141] Y. Ida, R. Settai, Y. Ota, F. Honda, Y. Ōnuki, Field-induced antiferromagnetism and upper critical field in pressure-induced superconductor CeRhIn $_{5}$, J. Phys. Soc. Jpn. 77 (2008) 084708.

[142] G. q. Zheng, K. Tanabe, T. Mito, S. Kawasaki, Y. Kitaoka, D. Aoki, Y. Haga, Y. Onuki, Unique spin dynamics and unconventional superconductivity in the layered heavy fermion compound CeIrIn ${ }_{5}: \mathrm{NQR}$ evidence, Phys. Rev. Lett. 86 (2001) 4664.

[143] W. Higemoto, A. Koda, R. Kadono, Y. Kawasaki, Y. Haga, D. Aoki, R. Settai, H. Shishido, Y. Ōnuki, $\mu$ SR studies on heavy fermion superconductors CeIrIn 5 and CeCoIn 5 , J. Phys. Soc. Jpn. 71 (2002) 1023.

[144] Y. Tokiwa, P. Gegenwart, D. Gnida, D. Kaczorowski, Quantum criticality near the upper critical field of $\mathrm{Ce}_{2} \mathrm{PdIn}_{8}$, Phys. Rev. B 84 (2011) 140507(R).

[145] J. K. Dong, H. Zhang, X. Qiu, B. Y. Pan, Y. F. Dai, T. Y. Guan, S. Y Zhou, D. Gnida, D. Kaczorowski, S. Y. Li, Field-induced quantum critical point and nodal superconductivity in the heavy-fermion superconductor $\mathrm{Ce}_{2}$ PdIn $_{8}$, Phys. Rev. X 1 (2011) 011010.

[146] H. Shishido, R. Settai, H. Harima, Y. Ōnuki, A drastic change of the Fermi surface at a critical pressure in $\mathrm{CeRhIn}_{5}$ : dHvA study under pressure, J. Phys. Soc. Jpn. 74 (2005) 1103

[147] A. Bianchi, R. Movshovich, N. Oeschler, P. Gegenwart, F. Steglich, J. D. Thompson, P. Pagliuso, J. L. Sarrao, First-order superconducting phase transition in CeCoIn 5 , Phys. Rev. Lett. 89 (2002) 137002.

[148] S. Kawasaki, T. Mito, Y. Kawasaki, G. q. Zheng, Y. Kitaoka, D. Aoki, Y. Haga, Y. Ōnuki, Gapless magnetic and quasiparticle excitations due to the coexistence of antiferromagnetism and superconductivity in CeRhIn ${ }_{5}$ : A study of ${ }^{115}$ In NQR under pressure, Phys. Rev. Lett. 91 (2003) 137001.

[149] A. Llobet, J. S. Gardner, E. G. Moshopoulou, J.-M. Mignot, M. Nicklas, W. Bao, N. O. Moreno, P. G. Pagliuso, I. N. Goncharenko, J. L. Sarrao, J. D. Thompson, Magnetic structure of $\mathrm{CeRhIn}_{5}$ as a function of pressure and temperature, Phys. Rev. B 69 (2004) 024403.

[150] N. Aso, K. Ishii, H. Yoshizawa, T. Fujiwara, Y. Uwatoko, G.-F. Chen, N. K. Sato, K. Miyake, Switching of magnetic ordering in $\mathrm{CeRhIn}_{5}$ under hydrostatic pressure, J. Phys. Soc. Jpn. 78 (2009) 073703.

[151] G. Knebel, D. Aoki, D. Braithwaite, B. Salce, J. Flouquet, Coexistence of antiferromagnetism and superconductivity in $\mathrm{CeRhIn}_{5}$ under high pressure and magnetic field, Phys. Rev. B 74 (2006) 020501(R).

[152] Y. Kohori, H. Taira, H. Fukazawa, T. Kohara, Y. Iwamoto, T. Matsumoto, M. B. Maple, ${ }^{115}$ In NQR studies of CeRhIn 5 and CeCoIn 5 under high pressure, J. Alloys Comp. 408-412 (2006) 51.

[153] T. Park, V. A. Sidorov, F. Ronning, J.-X. Zhu, Y. Tokiwa, H. Lee, E. D. Bauer, R. Movshovich, J. L. Sarrao, J. D. Thompson, Isotropic quantum scattering and unconventional superconductivity, Nature 456 (2008) 366.

[154] A. Bianchi, R. Movshovich, C. Capan, P. G. Pagliuso, J. L. Sarrao, Possible Fulde-Ferrell-Larkin-Ovchinnikov superconducting state in CeCoIn 5 , Phys. Rev. Lett. 91 (2003) 187004.

[155] L. D. Pham, T. Park, S. Maquilon, J. D. Thompson, Z. Fisk, Reversible tuning of the heavy-fermion ground state in $\mathrm{CeCoIn}_{5}$, Phys. Rev. Lett. 97 (2006) 056404.

[156] R. R. Urbano, B.-L. Young, N. J. Curro, J. D. Thompson, L. D Pham, Z. Fisk, Interacting antiferromagnetic droplets in quantum critical CeCoIn 5 , Phys. Rev. Lett. 99 (2007) 146402.

[157] S. Nair, O. Stockert, U. Witte, M. Nicklas, R. Schedler, K. Kiefer, J. D. Thompson, A. D. Bianchi, Z. Fisk, S. Wirth, F. Steglich, Magnetism and superconductivity driven by identical $4 f$ states in a heavy-fermion metal, Proc. Natl. Acad. Sci. 107 (2010) 9537.

[158] A. Bianchi, R. Movshovich, I. Vekhter, P. Pagliuso, J. L. Sarrao, Avoided antiferromagnetic order and quantum critical point in CeCoIn 5 , Phys. Rev. Lett. 91 (2003) 257001

[159] J. Paglione, M. A. Tanatar, D. G. Hawthorn, E. Boaknin, R. W. Hill, F. Ronning, M. Sutherland, L. Taillefer, C. Petrovic, P. C. Canfield,
Field-induced quantum critical point in $\mathrm{CeCoIn}_{5}$, Phys. Rev. Lett. 91 (2003) 246405

[160] S. Zaum, K. Grube, R. Schäfer, E. D. Bauer, J. D. Thompson, H. v. Löhneysen, Towards the identification of a quantum critical line in the $(p, B)$ phase diagram of $\mathrm{CeCoIn}_{5}$ with thermal-expansion measurements, Phys. Rev. Lett. 106 (2011) 087003.

[161] M. A. Tanatar, J. Paglione, C. Petrovic, L. Taillefer, Anisotropic violation of the Wiedemann-Franz law at a quantum critical point, Science 316 (2007) 1320

[162] B.-L. Young, R. R. Urbano, N. J. Curro, J. D. Thompson, J. L. Sarrao, A. B. Vorontsov, M. J. Graf, Microscopic evidence for field-induced magnetism in CeCoIn 5 , Phys. Rev. Lett. 98 (2007) 036402.

[163] G. Koutroulakis, M. D. Stewart, Jr., V. F. Mitrović, M. Horvatić, C. Berthier, G. Lapertot, J. Flouquet, Field evolution of coexisting superconducting and magnetic orders in CeCoIn ${ }_{5}$, Phys. Rev. Lett. 104 (2010) 087001

[164] M. Kenzelmann, T. Strässle, C. Niedermayer, M. Sigrist, B. Padmanabhan, M. Zolliker, A. D. Bianchi, R. Movshovich, E. D. Bauer, J. L. Sarrao, J. D. Thompson, Coupled superconducting and magnetic order in $\mathrm{CeCoIn}_{5}$, Science 321 (2008) 1652.

[165] S. Gerber, M. Bartkowiak, J. L. Gavilano, E. Ressouche, N. Egetenmeyer, C. Niedermayer, A. D. Bianchi, R. Movshovich, E. D. Bauer, J. D. Thompson, M. Kenzelmann, Switching of magnetic domains reveals spatially inhomogeneous superconductivity, Nature Phys. 10 (2014) 126.

[166] C. F. Miclea, M. Nicklas, D. Parker, K. Maki, J. L. Sarrao, J. D. Thompson, G. Sparn, F. Steglich, Pressure dependence of the FuldeFerrell-Larkin-Ovchinnikov state in CeCoIn ${ }_{5}$, Phys. Rev. Lett. 96 (2006) 117001

[167] Y. Tokiwa, R. Movshovich, F. Ronning, E. D. Bauer, P. Papin, A. D. Bianchi, J. F. Rauscher, S. M. Kauzlarich, Z. Fisk, Anisotropic effect of $\mathrm{Cd}$ and $\mathrm{Hg}$ doping on the Pauli limited superconductor $\mathrm{CeCoIn}_{5}$, Phys. Rev. Lett. 101 (2008) 037001.

[168] R. Hu, Y. Lee, J. Hudis, V. F. Mitrovic, C. Petrovic, Composition and field-tuned magnetism and superconductivity in $\mathrm{Nd}_{1-x} \mathrm{Ce}_{x} \mathrm{CoIn}_{5}$, Phys. Rev. B 77 (2008) 165129.

[169] S. Raymond, S. M. Ramos, D. Aoki, G. Knebel, V. P. Mineev, G. Lapertot, Magnetic order in $\mathrm{Ce}_{0.95} \mathrm{Nd}_{0.05} \mathrm{CoIn}_{5}$ : The Q-phase at zero magnetic field, J. Phys. Soc. Jpn. 83 (2014) 013707

[170] J. Paglione, T. A. Sayles, P.-C. Ho, J. R. Jeffries, M. B. Maple, Incoherent non-Fermi-liquid scattering in a Kondo lattice, Nature Phys. 3 (2013) 703.

[171] E. D. Bauer, Y. feng Yang, C. Capan, R. R. Urbano, C. F. Miclea, H. Sakai, F. Ronning, M. J. Graf, A. V. Balatsky, R. Movshovich, A. D. Bianchi, A. P. Reyes, P. L. Kuhns, J. D. Thompson, Z. Fisk, Electronic inhomogeneity in a Kondo lattice, Proc. Natl. Acad. Sci. 108 (2011) 6857.

[172] L. Shu, R. E. Baumbach, M. Janoschek, E. Gonzales, K. Huang, T. A. Sayles, J. Paglione, J. O’Brien, J. J. Hamlin, D. A. Zocco, P.-C. Ho, C. A. McElroy, M. B. Maple, Correlated electron state in $\mathrm{Ce}_{1-x} \mathrm{Yb}_{x} \mathrm{CoIn}_{5}$ stabilized by cooperative valence fluctuations, Phys. Rev. Lett. 106 (2011) 156403

[173] C. Capan, G. Seyfarth, D. Hurt, B. Prevost, S. Roorda, A. D. Bianchi, Z. Fisk, Wilson ratio in Yb-substituted $\mathrm{CeCoIn}_{5}$, Eur. Phys. Lett. 92 (2010) 47004

[174] M. Shimozawa, T. Watashige, S. Yasumoto, Y. Mizakami, M. Nakamura, H. Shishido, S. K. Gho, T. Terashima, T. Shibauchi, Y. Matsuda, Strong suppression of superconductivity by divalent Ytterbium Kondoholes in CeCoIn 5 , Phys. Rev. B 86 (2012) 144526.

[175] S. Jang, B. D. White, I. K. Lum, H. Kim, M. A. Tanatar, W. E. Straszheim, R. Prozorov, T. Keiber, F. Bridges, L. Shu, R. E. Baumbach, M. Janoschek, M. B. Maple, Resolution of the discrepancy between the variation of the physical properties of $\mathrm{Ce}_{1-x} \mathrm{Yb}_{x} \mathrm{CoIn}_{5}$ single crystals and thin films with Yb composition, Phil. Mag. 94 (2014) 4219

[176] C. H. Booth, T. Durakiewicz, C. Capan, D. Hurt, A. D. Bianchi, J. J. Joyce, Z. Fisk, Electronic structure and $f$-orbital occupancy in $\mathrm{Yb}$ substituted CeCoIn 5 , Phys. Rev. B 83 (2011) 235117.

[177] L. Dudy, J. D. Denlinger, L. Shu, M. Janoschek, J. W. Allen, M. B. Maple, $\mathrm{Yb}$ valence change in $\mathrm{Ce}_{1-x} \mathrm{Yb}_{x} \mathrm{CoIn}_{5}$ from spectroscopy and bulk properties, Phys. Rev. B 88 (2013) 165118

[178] C. Petrovic, S. L. Bud'ko, V. G. Kogan, P. C. Canfield, Effects of La 
substitution on the superconducting state of $\mathrm{CeCoIn}_{5}$, Phys. Rev. B 66 (2006) 054534.

[179] B. D. White, J. J. Hamlin, K. Huang, L. Shu, I. K. Lum, R. E. Baumbach, M. Janoschek, M. B. Maple, Insensitivity of the pressure dependences of characteristic energy scales in $\mathrm{Ce}_{1-x} R_{x} \operatorname{CoIn}_{5}(R=\mathrm{Yb}, \mathrm{Y}, \mathrm{Gd})$ to the electronic configuration of the rare-earth ion, Phys. Rev. B 86 (2012) 100502(R).

[180] T. Hu, Y. P. Singh, L. Shu, M. Janoschek, M. Dzero, M. B. Maple, C. C. Almasan, Non-Fermi liquid regimes with and without quantum criticality in $\mathrm{Ce}_{1-x} \mathrm{Yb}_{x} \mathrm{CoIn}_{5}$, Proc. Natl. Acad. Sci. USA 110 (2013) 7160.

[181] Y. P. Singh, D. J. Haney, X. Y. Huang, I. K. Lum, B. D. White, M. Dzero, M. B. Maple, C. C. Almasan, From local moment to mixed-valence regime in $\mathrm{Ce}_{1-x} \mathrm{Yb}_{x} \mathrm{CoIn}_{5}$ alloys, Phys. Rev. B 89 (2014) 115106.

[182] H. Kim, M. A. Tanatar, R. Flint, C. Petrovic, R. Hu, B. D. White, I. K. Lum, M. B. Maple, R. Prozorov, Nodal to nodeless superconducting energy-gap structure change concomitant with Fermi-surface reconstruction in the heavy-fermion compound $\mathrm{CeCoIn}_{5}$, Phys. Rev. Lett. 114 (2015) 027003.

[183] Y. P. Singh, D. J. Haney, X. Y. Huang, B. D. White, M. B. Maple, M. Dzero, C. C. Almasan, Pressure studies of the quantum critical alloy $\mathrm{Ce}_{0.93} \mathrm{Yb}_{0.07} \mathrm{CoIn}_{5}$, arXiv:1411.2524v1 [cond-mat.str-el].

[184] A. Polyakov, O. Ignatchik, B. Bergk, K. Götze, A. D. Bianchi, S. Blackburn, B. Prèvost, G. Seyfarth, M. Côté, D. Hurt, C. Capan, Z. Fisk, R. G. Goodrich, I. Sheikin, M. Richter, J. Wosnitza, Fermi-surface evolution in Yb-substituted CeCoIn 5 , Phys. Rev. B 85 (2012) 245119.

[185] O. Erten, R. Flint, P. Coleman, Molecular pairing and fully gapped superconductivity in Yb-doped CeCoIn 5 , Phys. Rev. Lett. 114 (2015) 027002.

[186] P. G. Pagliuso, R. Movshovich, A. D. Bianchi, M. Nicklas, N. O. Moreno, J. D. Thompson, M. F. Hundley, J. L. Sarrao, Z. Fisk, Multiple phase transitions in $\mathrm{Ce}(\mathrm{Rh}, \mathrm{Ir}, \mathrm{Co}) \mathrm{In}_{5}$, Physica B 312-313 (2002) 129.

[187] V. S. Zapf, E. J. Freeman, E. D. Bauer, J. Petricka, C. Sirvent, N. A. Frederick, R. P. Dickey, M. B. Maple, Coexistence of superconductivity and antiferromagnetism in $\mathrm{CeRh}_{1-x} \mathrm{Co}_{x} \mathrm{In}_{5}$, Phys. Rev. B 65 (2001) 014506.

[188] P. G. Pagliuso, C. Petrovic, R. Movshovich, D. Hall, M. F. Hundley, J. L. Sarrao, J. D. Thompson, Z. Fisk, Coexistence of magnetism and superconductivity in $\mathrm{CeRh}_{1-x} \mathrm{Ir}_{x} \mathrm{In}_{5}$, Phys. Rev. B 64 (2001) 100503(R).

[189] S. Ohira-Kawamura, H. Shishido, A. Yoshida, R. Okazaki, H. KawanoFurukawa, T. Shibauchi, H. Harima, Y. Matsuda, Competition between unconventional superconductivity and incommensurate antiferromagnetic order in $\mathrm{CeRh}_{1-x} \mathrm{Co}_{x} \mathrm{In}_{5}$, Phys. Rev. B 76 (2007) 132507.

[190] A. D. Christianson, A. Llobet, W. Bao, J. S. Gardner, I. P. Swainson, J. W. Lynn, J.-M. Mignot, K. Prokes, P. G. Pagliuso, N. O. Moreno, J. L. Sarrao, J. D. Thompson, A. H. Lacerda, Novel coexistence of superconductivity with two distinct magnetic orders, Phys. Rev. Lett. 95 (2005) 217002.

[191] G. qing Zheng, N. Yamaguchi, H. Kan, Y. Kitaoka, J. L. Sarrao, P. G. Pagliuso, N. O. Moreno, J. D. Thompson, Coexistence of antiferromagnetic order and unconventional superconductivity in heavyfermion $\mathrm{CeRh}_{1-x} \operatorname{Ir}_{x} \operatorname{In}_{5}$ compounds: Nuclear quadrupole resonance studies, Phys. Rev. B 70 (2004) 014511.

[192] H. Sakai, Y. Tokunaga, S. Kambe, F. Ronning, E. D. Bauer, J. D. Thompson, Coexistence of antiferromagnetism with superconductivity in $\mathrm{CePt}_{2} \mathrm{In}_{7}$ : Microscopic phase diagram determined by ${ }^{115} \mathrm{In}$ NMR and NQR, Phys. Rev. Lett. 112 (2014) 206401.

[193] M. Nicklas, V. A. Sidorov, H. A. Borges, P. G. Pagliuso, J. L. Sarrao, J. D. Thompson, Two superconducting phases in $\mathrm{CeRh}_{1-x} \mathrm{Ir}_{x} \mathrm{In}_{5}$, Phys. Rev. B 70 (2004) 020505(R)

[194] J. R. Jeffries, N. A. Frederick, E. D. Bauer, H. Kimura, V. S. Zapf, K.-D. Hof, T. A. Sayles, M. B. Maple, Superconductivity and nonFermi liquid behavior near antiferromagnetic quantum critical points in $\mathrm{CeRh}_{1-x} \mathrm{Co}_{x} \mathrm{In}_{5}$, Phys. Rev. B 72 (2005) 024551.

[195] S. K. Goh, J. Paglione, M. Sutherland, E. C. T. O'Farrell, C. Bergemann, T. A. Sayles, M. B. Maple, Fermi-surface reconstruction in $\mathrm{CeRh}_{1-x} \mathrm{Co}_{x} \mathrm{In}_{5}$, Phys. Rev. Lett. 101 (2008) 056402.

[196] P. Monthoux, D. Pines, G. G. Lonzarich, Superconductivity without phonons, Nature 450 (2007) 1177.

[197] J. H. Pixley, L. Deng, K. Ingersent, Q. Si, Pairing correlations near a Kondo-destruction quantum critical point, arXiv:1308.0839v2 [cond- mat.str-el].

[198] S. Kambe, H. Sakai, Y. Tokunaga, R. E. Walstedt, Quantum critical behavior in heavy-fermion superconductor CeIrIn ${ }_{5}$, Phys. Rev. B 82 (2010) 144503

[199] T. Shang, R. E. Baumbach, K. Gofryk, F. Ronning, Z. F. Weng, J. L. Zhang, X. Lu, E. D. Bauer, J. D. Thompson, H. Q. Yuan, CeIrIn 5 : Superconductivity on a magnetic instability, Phys. Rev. B 89 (2014) 041101(R).

[200] C. Stock, C. Broholm, J. Hudis, H. J. Kang, C. Petrovic, Spin resonance in the $d$-wave superconductor $\mathrm{CeCoIn}_{5}$, Phys. Rev. Lett. 100 (2008) 087001 .

[201] G. Yu, Y. Li, E. M. Motoyama, M. Greven, A universal relationship between magnetic resonance and superconducting gap in unconventional superconductors, Nat. Phys. 5 (2009) 873.

[202] J. S. Van Dyke, F. Massee, M. P. Allan, J. C. S. Davis, C. Petrovic, D. K. Morr, Direct evidence for a magnetic $f$-electron-mediated pairing mechanism of heavy-fermion superconductivity in $\mathrm{CeCoIn}_{5}$, Proc. Natl. Acad. Sci. 111 (2014) 11663.

[203] Y. Mizukami, H. Shishido, T. Shibauchi, M. Shimozawa, S. Yasumoto, D. Watanabe, M. Yamashita, H. Ikeda, T. Terashima, H. Kontani, Y. Matsuda, Extremely strong-coupling superconductivity in artificial twodimensional Kondo lattices, Nature Phys. 7 (2011) 849.

[204] D. J. Scalapino, A common thread: The pairing interaction for unconventional superconductors, Rev. Mod. Phys. 84 (2012) 1383.

[205] N. Kimura, I. Bonalde, Non-centrosymmetric heavy-fermion superconductors, in: E. Bauer, M. Sigrist (Eds.), Non-Centrosymmetric Superconductors: Introduction and Overview, Springer-Verlag, 2012, Ch. 2.

[206] E. Bauer, G. Hilscher, H. Michor, C. Paul, E. W. Scheidt, A. Gribanov, Y. Seropegin, H. Noël, M. Sigrist, P. Rogl, Heavy fermion superconductivity and magnetic order in noncentrosymmetric $\mathrm{CePt}_{3} \mathrm{Si}$, Phys. Rev. Lett. 92 (2004) 027003.

[207] N. Kimura, K. Ito, K. Saitoh, Y. Umeda, H. Aoki, T. Terashima, Pressure-induced superconductivity in noncentrosymmetric heavyfermion $\mathrm{CeRhSi}_{3}$, Phys. Rev. Lett. 95 (2005) 247004.

[208] I. Sugitani, Y. Okuda, H. Shishido, T. Yamada, A. Thamizhavel, E. Yamamoto, T. D. Matsuda, Y. Haga, T. Takeuchi, R. Settai, Y. Ōnuki, Pressure-induced heavy-fermion superconductivity in antiferromagnet $\mathrm{CeISSi}_{3}$ without inversion symmetry, J. Phys. Soc. Jpn. 75 (2006) 043703.

[209] R. Settai, I. Sugitani, Y. Okuda, A. Thamizhavel, M. Nakashima, Y. Ōnuki, H. Harima, Pressure-induced superconductivity in $\mathrm{CeCoGe}_{3}$ without inversion symmetry, J. Mag. Mag. Mater. 310 (2007) 844.

[210] T. Yasuda, H. Shishido, T. Ueda, S. Hashimoto, R. Settai, T. Takeuchi, T. D. Matsuda, Y. Haga, Y. Ōnuki, Superconducting property in $\mathrm{CePt}_{3} \mathrm{Si}$ under pressure, J. Phys. Soc. Jpn. 73 (2004) 1657.

[211] E. Bauer, H. Kaldarar, A. Prokofiev, E. Royanian, A. Amato, J. Sereni, W. Brämer-Escamilla, I. Bonalde, Heavy fermion superconductivity and antiferromagnetic ordering in $\mathrm{CePt}_{3} \mathrm{Si}$ without inversion symmetry, J. Phys. Soc. Jpn. 76 (2007) 051009.

[212] T. Kawai, H. Muranaka, M.-A. Measson, T. Shimoda, Y. Doi, T. D. Matsuda, Y. Haga, G. Knebel, G. Lapertot, D. Aoki, J. Flouquet, T. Takeuchi, R. Settai, Y. Ōnuki, Magnetic and superconducting properties of $\mathrm{CeTX}_{3}$ (T: Transition metal and X: Si and Ge) with non-centrosymmetric crystal structure, J. Phys. Soc. Jpn. 77 (2008) 064716.

[213] N. Kimura, K. Ito, H. Aoki, S. Uji, T. Terashima, Extremely high upper critical magnetic field of the noncentrosymmetric heavy fermion superconductor CeRhSi ${ }_{3}$, Phys. Rev. Lett. 98 (2007) 197001.

[214] R. Settai, Y. Miyauchi, T. Takeuchi, F. Lévy, I. Sheikin, Y. Ōnuki, Huge upper critical field and electronic instability in pressure-induced superconductor $\mathrm{CeIrSi}_{3}$ without inversion symmetry in the crystal structure, J. Phys. Soc. Jpn. 77 (2008) 073705.

[215] A. P. Pikul, D. Kaczorowski, T. Plackowski, A. Czopnik, H. Michor, E. Bauer, G. Hilscher, P. Rogl, Y. Grin, Kondo behavior in antiferromagnetic $\mathrm{CeNiGe}_{3}$, Phys. Rev. B 67 (2003) 224417.

[216] M. Nakashima, K. Tabata, A. Thamizhavel, T. C. Kobayashi, M. Hedo, Y. Uwatoko, K. Shimizu, R. Settai, Y. Ōnuki, High-pressure effect on the electronic state in $\mathrm{CeNiGe}_{3}$ : pressure-induced superconductivity, J. Phys.: Condens. Matter 16 (2004) L255.

[217] H. Kotegawa, K. Takeda, T. Miyoshi, S. Fukushima, H. Hidaka, T. C. Kobayashi, T. Akazawa, Y. Ohishi, M. Nakashima, A. Thamizhavel, R. Settai, Y. Ōnuki, Pressure-induced superconductivity emerging from 
antiferromagnetic phase in $\mathrm{CeNiGe}_{3}$, J. Phys. Soc. Jpn. 75 (2006) 044713.

[218] L. Durivault, F. Bourée, B. Chevalier, G. André, J. Etourneau, Magnetic ordering in the ternary germanide $\mathrm{Ce}_{2} \mathrm{Ni}_{3} \mathrm{Ge}_{5}$ as studied by neutron powder diffraction, J. Mag. Mag. Mater. 246 (2002) 366.

[219] Z. Hossain, S. Hamashima, K. Umeo, T. Takabatake, C. Geibel, F. Steglich, Antiferromagnetic transitions in the Kondo lattice system $\mathrm{Ce}_{2} \mathrm{Ni}_{3} \mathrm{Ge}_{5}$, Phys. Rev. B 62 (2000) 8950.

[220] M. Nakashima, H. Kohara, A. Thamizhavel, T. D. Matsuda, Y. Haga, M. Hedo, Y. Uwatoko, R. Settai, Y. Ōnuki, A change of electronic state tuned by pressure: pressure-induced superconductivity of the antiferromagnet $\mathrm{Ce}_{2} \mathrm{Ni}_{3} \mathrm{Ge}_{5}$, J. Phys.: Condens. Matter 17 (2005) 4539.

[221] D. Aoki, Y. Haga, T. D. Matsuda, N. Tateiwa, S. Ikeda, Y. Homma, H. Sakai, Y. Shiokawa, E. Yamamoto, A. Nakamura, R. Settai, Y. Ōnuki, Unconventional heavy-fermion superconductivity of a new transuranium compound $\mathrm{NpPd}_{5} \mathrm{Al}_{2}$, J. Phys. Soc. Jpn. 76 (2007) 063701.

[222] F. Honda, M.-A. Measson, Y. Nakano, N. Yoshitani, E. Yamamoto, Y. Haga, T. Takeuchi, H. Yamagami, K. Shimizu, R. Settai, Y. Ōnuki, Pressure-induced superconductivity in antiferromagnet $\mathrm{CePd}_{5} \mathrm{Al}_{2}, \mathrm{~J}$. Phys. Soc. Jpn. 77 (2008) 043701.

[223] G. Knebel, D. Braithwaite, P. C. Canfield, G. Lapertot, J. Flouquet, Electronic properties of $\mathrm{CeIn}_{3}$ under high pressure near the quantum critical point, Phys. Rev. B 65 (2001) 024425.

[224] K. N. Yang, M. B. Maple, L. E. DeLong, J. G. Huber, A. Junod, Lowtemperature heat-capacity study of the $\mathrm{U}_{6} X(X \equiv \mathrm{Mn}, \mathrm{Fe}, \mathrm{Co}, \mathrm{Ni})$ compounds, Phys. Rev. B 39 (1989) 151.

[225] L. E. DeLong, J. G. Huber, K. N. Yang, M. B. Maple, Observation of high-field superconductivity of a strongly interacting Fermi liquid in $\mathrm{U}_{6} \mathrm{Fe}$, Phys. Rev. Lett. 51 (1983) 312.

[226] N. C. Baenziger, R. E. Rundle, A. I. Snow, A. S. Wilson, Compounds of uranium with the transition metals of the first long period, Acta Cryst. 3 (1950) 34 .

[227] B. T. Matthias, C. W. Chu, E. Corenzwit, D. Wohlleben, Ferromagnetism and superconductivity in uranium compounds, Proc. Natl. Acad. Sci. 64 (1969) 459.

[228] G. P. Meisner, A. L. Giorgi, A. C. Lawson, G. R. Stewart, J. O. Willis, M. S. Wire, J. L. Smith, $\mathrm{U}_{2} \mathrm{PtC}_{2}$ and systematics of heavy fermions, Phys. Rev. Lett. 53 (1984) 1829.

[229] A. M. Mounce, H. Yasuoka, G. Koutroulakis, N. Ni, E. D. Bauer, F. Ronning, J. D. Thompson, Evidence for spin-triplet superconductivity in $\mathrm{U}_{2} \mathrm{PtC}_{2}$ from ${ }^{195} \mathrm{Pt}$ NMR, arXiv:1408.1969v1 [cond-mat.supr-con].

[230] L. Glémot, J. P. Brison, J. Flouquet, A. I. Buzdin, I. Sheikin, D. Jaccard, C. Thessieu, F. Thomas, Pressure dependence of the upper critical field of the heavy fermion superconductor $\mathrm{UBe}_{13}$, Phys. Rev. Lett. 82 (1999) 169.

[231] D. W. Cooke, R. H. Heffner, R. L. Hutson, M. E. Schillaci, J. L. Smith, J. O. Willis, D. E. MacLaughlin, C. Boekema, R. L. Lichti, A. B. Denison, J. Oostens, Muon spin relaxation and Knight shift in the heavyfermion superconductor $\mathrm{UPt}_{3}$, Hyperfine Interactions 31 (1986) 425.

[232] G. Aeppli, E. Bucher, C. Broholm, J. K. Kjems, J. Baumann, J. Hufnagl, Magnetic order and fluctuations in superconducting $\mathrm{UPt}_{3}$, Phys. Rev Lett. 60 (1988) 615.

[233] R. A. Fisher, S. Kim, B. F. Woodfield, N. E. Phillips, L. Taillefer, K. Hasselbach, J. Flouquet, A. L. Giorgi, J. L. Smith, Specific heat of UPt 3 : Evidence for unconventional superconductivity, Phys. Rev. Lett. 62 (1989) 1411.

[234] A. Huxley, P. Rodíere, D. M. Paul, N. van Dijk, R. Cubitt, J. Flouquet, Realignment of the flux-line lattice by a change in the symmetry of superconductivity in $\mathrm{UPt}_{3}$, Nature 406 (2000) 160.

[235] J. P. Brison, N. Keller, A. Vernière, P. Lejay, L. Schmidt, A. Buzdin, J. Flouquet, S. R. Julian, G. G. Lonzarich, Anisotropy of the upper critical field in $\mathrm{URu}_{2} \mathrm{Si}_{2}$ and FFLO state in antiferromagnetic superconductors, Physica C 250 (1995) 128.

[236] C. Geibel, C. Schank, S. Thies, H. Kitazawa, C. D. Bredl, A. Bohm, M. Rau, A. Grauel, R. Caspary, R. Helfrich, U. Ahlheim, G. Weber, F. Steglich, Heavy-fermion superconductivity at $T_{c}=2 \mathrm{~K}$ in the antiferromagnet $\mathrm{UPd}_{2} \mathrm{Al}_{3}$, Z. Phys. B 84 (1991) 1

[237] R. Caspary, P. Hellmann, M. Keller, G. Sparn, C. Wassilew, R. Köhler, C. Geibel, C. Schank, F. Steglich, N. E. Phillips, Unusual groundstate properties of $\mathrm{UPd}_{2} \mathrm{Al}_{3}$ : Implications for the coexistence of heavyfermion superconductivity and local-moment antiferromagnetism, Phys.
Rev. Lett. 71 (1993) 2146.

[238] A. Ishiguro, A. Sawada, Y. Inada, J. Kimura, M. Suzuki, N. Sato, T. Komatsubara, Anomalous susceptibility of $\mathrm{UPd}_{2} \mathrm{Al}_{3}$ near the upper critical field, J. Phys. Soc. Jpn. 64 (1995) 378.

[239] T. Watanabe, K. Izawa, Y. Kasahara, Y. Haga, Y. Onuki, P. Thalmeier, K. Maki, Y. Matsuda, Superconducting gap function in antiferromagnetic heavy-fermion $\mathrm{UPd}_{2} \mathrm{Al}_{3}$ probed by angle-resolved magnetothermal transport measurements, Phys. Rev. B 70 (2004) 184502.

[240] H. Tou, Y. Kitaoka, T. Kamatsuka, K. Asayama, C. Geibel, F. Steglich, S. Süllow, J. A. Mydosh, NMR/NQR studies of U-123 type heavy fermion compounds, Physica B 230-232 (1997) 360.

[241] C. Geibel, S. Thies, D. Kaczorowski, A. Mehner, A. Grauel, B. Seidel, U. Ahlheim, R. Helfrich, K. Petersen, C. D. Bredl, F. Steglich, A new heavy-fermion superconductor: $\mathrm{UNi}_{2} \mathrm{Al}_{3}$, Z. Phys. B 83 (1991) 305.

[242] N. Sato, N. Koga, T. Komatsubara, Anisotropy of upper critical magnetic fields in the heavy fermion superconductor $\mathrm{UNi}_{2} \mathrm{Al}_{3}$, J. Phys. Soc. Jpn. 65 (1996) 1555.

[243] Y. Ōnuki, I. Ukon, S. W. Yun, I. Umehara, K. Satoh, T. Fukuhara, H. Sato, S. Takayanagi, M. Shikama, A. Ochiai, Magnetic and electrical properties of U-Ge intermetallic compounds, J. Phys. Soc. Jpn. 61 (1992) 293.

[244] A. Huxley, I. Sheikin, E. Ressouche, N. Kernavanois, D. Braithwaite, R. Calemczuk, J. Flouquet, $\mathrm{UGe}_{2}$ : A ferromagnetic spin-triplet superconductor, Phys. Rev. B 63 (2001) 144519.

[245] S. S. Saxena, P. Agarwal, K. Ahilan, F. M. Grosche, R. K. W. Haselwimmer, M. J. Steiner, E. Pugh, I. R. Walker, S. R. Julian, P. Monthoux, G. G. Lonzarich, A. Huxley, I. Sheikin, D. Braithwaite, J. Flouquet, Superconductivity on the border of itinerant-electron ferromagnetism in $\mathrm{UGe}_{2}$, Nature 406 (2000) 587.

[246] N. Tateiwa, T. C. Kobayashi, K. Hanazono, K. Amaya, Y. Haga, R. Settai, Y. Onuki, Pressure-induced superconductivity in a ferromagnet $\mathrm{UGe}_{2}$, J. Phys.: Condens. Matter 13 (2001) L17.

[247] D. Aoki, A. Huxley, E. Ressouche, D. Braithwaite, J. Flouquet, J.-P. Brison, E. Lhotel, C. Paulsen, Coexistence of superconductivity and ferromagnetism in URhGe, Nature 413 (2001) 613.

[248] F. Hardy, A. D. Huxley, p-wave superconductivity in the ferromagnetic superconductor URhGe, Phys. Rev. Lett. 94 (2005) 247006.

[249] N. T. Huy, A. Gasparini, D. E. de Nijs, Y. Huang, J. C. P. Klaasse, T. Gortenmulder, A. de Visser, A. Hamann, T. Görlach, H. v. Löhneysen, Superconductivity on the border of weak itinerant ferromagnetism in UCoGe, Phys. Rev. Lett. 99 (2007) 067006.

[250] N. T. Huy, D. E. de Nijs, Y. K. Huang, A. de Visser, Unusual upper critical field of the ferromagnetic superconductor UCoGe, Phys. Rev. Lett. 100 (2008) 077002.

[251] E. Slooten, T. Naka, A. Gasparini, Y. K. Huang, A. de Visser, Enhancement of superconductivity near the ferromagnetic quantum critical point in UCoGe, Phys. Rev. Lett. 103 (2009) 097003.

[252] C. Paulsen, D. J. Hykel, K. Hasselbach, D. Aoki, Observation of the Meissner-Ochsenfeld effect and the absence of the Meissner state in UCoGe, Phys. Rev. Lett. 109 (2012) 237001.

[253] T. Hattori, Y. Ihara, Y. Nakai, K. Ishida, Y. Tada, S. Fujimoto, N. Kawakami, E. Osaki, K. Deguchi, N. K. Sato, I. Satoh, Superconductivity induced by longitudinal ferromagnetic fluctuations in UCoGe, Phys. Rev. Lett. 108 (2012) 066403.

[254] T. Akazawa, H. Hidaka, T. Fujiwara, T. C. Kobayashi, E. Yamamoto, Y. Haga, R. Settai, Y. Ōnuki, Pressure-induced superconductivity in ferromagnetic UIr without inversion symmetry, J. Phys.: Condens. Matter 16 (2004) L29.

[255] T. Akazawa, H. Hidaka, H. Kotegawa, T. C. Kobayashi, T. Fujiwara, E. Yamamoto, Y. Haga, R. Settai, Y. Ōnuki, Pressure-induced superconductivity in UIr, J. Phys. Soc. Jpn. 73 (2004) 3129.

[256] E. E. M. Chia, M. B. Salamon, H. Sugawara, H. Sato, Probing the superconducting gap symmetry of $\mathrm{PrOs}_{4} \mathrm{Sb}_{12}$ : A penetration depth study, Phys. Rev. Lett. 91 (2003) 247003.

[257] K. Izawa, Y. Nakajima, J. Goryo, Y. Matsuda, S. Osaki, H. Sugawara, H. Sato, P. Thalmeier, K. Maki, Multiple superconducting phases in new heavy fermion superconductor $\mathrm{PrOs}_{4} \mathrm{Sb}_{12}$, Phys. Rev. Lett. 90 (2003) 117001

[258] R. Vollmer, A. Faißt, C. Pfleiderer, H. v. Löhneysen, E. D. Bauer, P.-C. Ho, V. Zapf, M. B. Maple, Low-temperature specific heat of the heavyfermion superconductor $\mathrm{PrOs}_{4} \mathrm{Sb}_{12}$, Phys. Rev. Lett. 90 (2003) 057001. 
[259] A. Sakai, S. Nakatsuji, Kondo effects and multipolar order in the cubic $\operatorname{Pr} T r_{2} \mathrm{Al}_{20}(\operatorname{Tr}=\mathrm{Ti}, \mathrm{V})$, J. Phys. Soc. Jpn. 80 (2011) 063701

[260] A. Sakai, K. Kuga, S. Nakatsuji, Superconductivity in the ferroquadrupolar state in the quadrupolar Kondo lattice $\operatorname{PrTi}_{2} \mathrm{Al}_{20}$, J. Phys. Soc. Jpn. 81 (2012) 083702.

[261] K. Kuga, Y. Karaki, Y. Matsumoto, Y. Machida, S. Nakatsuji, Superconducting properties of the non-Fermi-liquid system $\beta$-YbAlB 4 , Phys. Rev. Lett. 101 (2008) 137004.

[262] Y. Haga, H. Sakai, S. Kambe, Recent advances in the $5 f$-relevant electronic states and unconventional superconductivity of actinide compounds, J. Phys. Soc. Jpn. 76 (2007) 051012

[263] E. D. Bauer, J. D. Thompson, J. L. Sarrao, L. A. Morales, F. Wastin, J. Rebizant, J. C. Griveau, P. Javorsky, P. Boulet, E. Colineau, G. H. Lander, G. R. Stewart, Structural tuning of unconventional superconductivity in ${\mathrm{Pu} M \mathrm{Ga}_{5}}_{(}(M=\mathrm{Co}, \mathrm{Rh})$, Phys. Rev. Lett. 93 (2004) 147005.

[264] P. Javorský, E. Colineau, F. Wastin, F. Jutier, J.-C. Griveau, P. Boulet, R. Jardin, J. Rebizant, Specific heat and anisotropy of the nonconventional superconductors PuCoGa 5 and PuRhGa5, Phys. Rev. B 75 (2007) 184501.

[265] B. S. Chandrasekhar, J. K. Hulm, The electrical resistivity and superconductivity of some uranium alloys and compounds, J. Phys. Chem. Solids 7 (1958) 259.

[266] T. Trappmann, H. v. Löhneysen, L. Taillefer, Pressure dependence of the superconducting phases in $\mathrm{UPt}_{3}$, Phys. Rev. B 43 (1991) 13714.

[267] R. H. Heffner, M. R. Norman, Heavy fermion superconductivity, Comments on Condensed Matter Physics 17 (1996) 361.

[268] S. E. Lambert, Y. Dalichaouch, M. B. Maple, J. L. Smith, Z. Fisk, Superconductivity under pressure in $\left(\mathrm{U}_{1-x} \mathrm{Th}_{x}\right) \mathrm{Be}_{13}$ : Evidence for two superconducting states, Phys. Rev. Lett. 57 (1986) 1619.

[269] T. F. Rosenbaum, The double transition in thoriated $\mathrm{UBe}_{13}$, in: P. Kumar (Ed.), Superconductivity Review, Vol. 2, Gordon and Breach, 1998, p. 257

[270] H. H. Hill, in: W. H. Miner (Ed.), Plutonium 1970 and Other Actinides, AIME, 1970.

271] A. Grauel, A. Böhm, H. Fischer, C. Geibel, R. Köhler, R. Modler, C. Schank, F. Steglich, G. Weber, T. Komatsubara, N. Sato, Tetravalency and magnetic phase diagram in the heavy-fermion superconductor $\mathrm{UPd}_{2} \mathrm{Al}_{3}$, Phys. Rev. B Rapid Comm. 46 (1992) 5818.

[272] S. Süllow, B. Becker, A. de Visser, M. Mihalik, G. J. Nieuwenhuys, A. A. Menovsky, J. A. Mydosh, Magnetic anisotropy of singlecrystalline $\mathrm{UNi}_{2} \mathrm{Al}_{3}$, J. Phys.: Condens. Matter 9 (1997) 913.

[273] A. Krimmel, P. Fischer, B. Roessli, H. Maletta, C. Geibel, C. Schank, A. Grauel, A. Loidl, F. Steglich, Neutron diffraction study of the heavy fermion superconductors $\mathrm{UM}_{2} \mathrm{Al}_{3}(\mathrm{M}=\mathrm{Pd}, \mathrm{Ni})$, Z. Phys. B 86 (1992) 161.

[274] A. Schröder, J. G. Lussier, B. D. Gaulin, J. D. Garrett, W. J. L. Buyers, L. Rebelsky, S. M. Shapiro, Incommensurate magnetic order in the heavy fermion superconductor $\mathrm{UNi}_{2} \mathrm{Al}_{3}$, Phys. Rev. Lett. 72 (1994) 136

[275] J. G. Lussier, M. Mao, A. Schröder, J. D. Garrett, B. D. Gaulin, S. M Shapiro, W. J. L. Buyers, Neutron-scattering study of incommensurate magnetic order in the heavy-fermion superconductor $\mathrm{UNi}_{2} \mathrm{Al}_{3}$, Phys. Rev. B 56 (1997) 11749

[276] Y. Dalichaouch, M. C. de Andrade, M. B. Maple, Superconducting and magnetic properties of the heavy-fermion compounds $\mathrm{UT}_{2} \mathrm{Al}_{3}(\mathrm{~T}=\mathrm{Ni}$, Pd), Phys. Rev. B Rapid Comm. 46 (1992) 8671

[277] N. Tateiwa, N. Sato, T. Komatsubara, Heat-capacity investigation on magnetism in $\mathrm{UNi}_{2} \mathrm{Al}_{3}$, Phys. Rev. B 58 (1998) 11131.

[278] P. Link, D. Jaccard, C. Geibel, C. Wassilew, F. Steglich, The heavyfermion superconductor $\mathrm{UPd}_{2} \mathrm{Al}_{3}$ at very high pressure, J. Phys. Cond. Matter 7 (1995) 373

[279] C. Wassilew, B. Kirsch, G. Sparn, C. Schank, C. Geibel, F. Steglich, Pressure dependence of the resistivity of $\mathrm{UNi}_{2} \mathrm{Al}_{3}$ and $\mathrm{UPd}_{2} \mathrm{Al}_{3}$, Physica B $199-200$ (1994) 162

[280] K. Ishida, D. Ozaki, T. Kamatsuka, H. Tou, M. Kyogaku, Y. Kitaoka, N. Tateiwa, N. K. Sato, N. Aso, C. Geibel, F. Steglich, Spin-triplet superconductivity in $\mathrm{UNi}_{2} \mathrm{Al}_{3}$ revealed by the ${ }^{27} \mathrm{Al}$ Knight shift measurement, Phys. Rev. Lett. 89 (2002) 037002.

[281] M. Jourdan, M. Huth, H. Adrian, Superconductivity mediated by spin fluctuations in the heavy-fermion compound $\mathrm{UPd}_{2} \mathrm{Al}_{3}$, Nature 398 (1999) 47.

[282] P. H. Frings, J. J. M. Franse, F. R. de Boer, A. Menovsky, Magnetic properties of $\mathrm{U}_{x} \mathrm{Pt}_{y}$ compounds, J. Mag. Mat. Mater. 31-34 (1983) 240 .

[283] J. W. Chen, S. E. Lambert, M. B. Maple, Z. Fisk, J. L. Smith, G. R. Stewart, J. O. Willis, Upper critical magnetic field of the heavy-fermion superconductor $\mathrm{UPt}_{3}$, Phys. Rev. B 30 (1984) 1583.

[284] P. E. Sulewski, A. J. Sievers, M. B. Maple, M. S. Torikachvili, J. L. Smith, Z. Fisk, Far-infrared absorptivity of $\mathrm{UPt}_{3}$, Phys. Rev. B 38 (1988) 5338.

[285] L. Taillefer, R. Newbury, G. G. Lonzarich, Z. Fisk, J. L. Smith, Direct observation of heavy quasiparticles in $\mathrm{UPt}_{3}$ via the dHvA effect, J. Mag. Mag. Mat. 63-64 (1987) 372.

[286] L. Taillefer, G. G. Lonzarich, Heavy-fermion quasiparticles in $\mathrm{UPt}_{3}$, Phys. Rev. Lett. 60 (1988) 1570.

[287] H. Tou, Y. Kitaoka, K. Asayama, N. Kimura, Y. Ōnuki, E. Yamamoto, K. Maezawa, Odd-parity superconductivity with parallel spin pairing in $\mathrm{UPt}_{3}$ : Evidence from ${ }^{195} \mathrm{Pt}$ Knight shift study, Phys. Rev. Lett. 77 (1996) 1374.

[288] K. Hasselbach, L. Taillefer, J. Flouquet, Critical point in the superconducting phase diagram of $\mathrm{UPt}_{3}$, Phys. Rev. Lett. 63 (1989) 93.

[289] R. Joynt, L. Taillefer, The superconducting phases of UPt 3 , Rev. Mod. Phys. 74 (2002) 235

[290] B. S. Shivaram, T. F. Rosenbaum, D. G. Hinks, Unusual angular and temperature dependence of the upper critical field in $\mathrm{UPt}_{3}$, Phys. Rev. Lett. 57 (1986) 1259

[291] S. M. Hayden, L. Taillefer, C. Vettier, J. Flouquet, Antiferromagnetic order in $\mathrm{UPt}_{3}$ under pressure: Evidence for a direct coupling to superconductivity, Phys. Rev. B 46 (1992) 8675.

[292] J. P. Brison, N. Keller, P. Lejay, A. Huxley, L. Schmidt, A. Buzdin, N. R. Bernhoeft, I. Mineev, A. N. Stepanov, J. Flouquet, D. Jaccard, S. R. Julian, G. Lonzarich, Very low temperature properties of heavy fermion materials, Physica B 199-200 (1994) 70.

[293] J. A. Sauls, The order parameter for the superconducting phases of $\mathrm{UPt}_{3}$, Adv. Phys. 43 (1994) 113.

[294] J. D. Strand, D. J. Bahr, D. J. V. Harlingen, J. P. Davis, W. J. Gannon, W. P. Halperin, The transition between real and complex superconducting order parameter phases in $\mathrm{UPt}_{3}$, Science 328 (2010) 1368.

[295] E. R. Schemm, W. J. Gannon, C. M. Wishne, W. P. Halperin, A. Kapitulnik, Observation of broken time-reversal symmetry in the heavy-fermion superconductor $\mathrm{UPt}_{3}$, Science 345 (2014) 190.

[296] T. T. M. Palstra, A. A. Menovsky, J. A. Mydosh, Anisotropic electrical resistivity of the magnetic heavy-fermion superconductor $\mathrm{URu}_{2} \mathrm{Si}_{2}$, Phys. Rev. B 33 (1986) 6527

[297] D. A. Bonn, J. D. Garrett, T. Timusk, Far-infrared properties of $\mathrm{URu}_{2} \mathrm{Si}_{2}$, Phys. Rev. Lett. 61 (1988) 1305.

298] P. Aynajian, E. H. da Silva Neto, C. V. Parker, Y. Huang, A. Pasupathy, J. Mydosh, A. Yazdani, Visualizing the formation of the Kondo lattice and the hidden order in $\mathrm{URu}_{2} \mathrm{Si}_{2}$, Proc. Natl. Sci. Acad. 107 (2010) 10383

[299] A. R. Schmidt, M. H. Hamidian, P. Wahl, F. Meier, A. V. Balatsky, J. D. Garrett, T. J. Williams, G. M. Luke, J. C. Davis, Imaging the Fano lattice to 'hidden order' transition in $\mathrm{URu}_{2} \mathrm{Si}_{2}$, Nature 465 (2010) 570

[300] C. Broholm, J. K. Kjems, W. J. L. Buyers, P. Matthew, T. T. M. Palstra, A. A. Menovsky, J. A. Mydosh, Magnetic excitations and ordering in the heavy-electron superconductor $\mathrm{URu}_{2} \mathrm{Si}_{2}$, Phys. Rev. Lett. 58 (1987) 1467.

[301] P. G. Niklowitz, C. Pfleiderer, T. Keller, M. Vojta, Y.-K. Huang, J. A. Mydosh, Parasitic small-moment antiferromagnetism and nonlinear coupling of hidden order and antiferromagnetism in $\mathrm{URu}_{2} \mathrm{Si}_{2}$ observed by Larmor diffraction, Phys. Rev. Lett. 104 (2010) 106406.

[302] J. A. Mydosh, P. M. Oppeneer, Colloquium: Hidden order, superconductivity, and magnetism: The unsolved case of $\mathrm{URu}_{2} \mathrm{Si}_{2}$, Rev. Mod. Phys. 83 (2011) 1301.

[303] G. Motoyama, N. Yokoyama, A. Sumiyama, Y. Oda, Electrical resistivity and thermal expansion measurements of $\mathrm{URu}_{2} \mathrm{Si}_{2}$ under pressure, $\mathrm{J}$. Phys. Soc. Jpn. 77 (2008) 123710.

[304] N. P. Butch, J. R. Jeffries, S. Chi, J. B. Leão, J. W. Lynn, M. B. Maple, Antiferromagnetic critical pressure in $\mathrm{URu}_{2} \mathrm{Si}_{2}$ under hydrostatic conditions, Phys. Rev. B 82 (2010) 060408(R).

[305] H. Amitsuka, M. Sato, N. Metoki, M. Yokoyama, K. Kuwahara, T. Sakakibara, H. Morimoto, S. Kawarazaki, Y. Miyako, J. A. Mydosh, Effect of pressure on tiny antiferromagnetic moment in the heavyelectron compound $\mathrm{URu}_{2} \mathrm{Si}_{2}$, Phys. Rev. Lett. 83 (1999) 5114 
[306] M. Yokoyama, H. Amitsuka, K. Tenya, K. Watanabe, S. Kawarazaki, H. Yoshizawa, J. A. Mydosh, Competition between hidden order and antiferromagnetism in $\mathrm{URu}_{2} \mathrm{Si}_{2}$ under uniaxial stress studied by neutron scattering, Phys. Rev. B 72 (2005) 214419

[307] R. Okazaki, M. Shimozawa, H. Shishido, M. Konczykowski, Y. Haga T. D. Matsuda, E. Yamamoto, Y. Ōnuki, Y. Yanase, T. Shibauchi, Y. Matsuda, Anomalous temperature dependence of lower critical field in ultraclean $\mathrm{URu}_{2} \mathrm{Si}_{2}$, J. Phys. Soc. Jpn. 79 (2010) 084705.

[308] K. H. Kim, N. Harrison, M. Jaime, G. S. Boebinger, J. A. Mydosh, Magnetic-field-induced quantum critical point and competing order parameters in $\mathrm{URu}_{2} \mathrm{Si}_{2}$, Phys. Rev. Lett. 91 (2003) 256401.

[309] Y. Dalichaouch, M. B. Maple, M. S. Torikachvili, A. L. Giorgi, Ferromagnetic instability in the heavy-electron compound $\mathrm{URu}_{2} \mathrm{Si}_{2}$ doped with Re or Tc, Phys. Rev. B 39 (1989) 2423.

[310] Y. Dalichaouch, M. B. Maple, R. P. Guertin, M. V. Kuric, M. S Torikachvili, A. L. Giorgi, Ferromagnetism and heavy electron behavior in $\mathrm{URu}_{2-x} \mathrm{M}_{x} \mathrm{Si}_{2}(\mathrm{M}=\mathrm{Re}, \mathrm{Tc}$ and $\mathrm{Mn})$, Physica B 163 (1990) 113.

[311] Y. Dalichaouch, M. B. Maple, J. W. Chen, T. Kohara, C. Rossel, M. S. Torikachvili, A. L. Giorgi, Effect of transition-metal substitutions on competing electronic transitions in the heavy-electron compound $\mathrm{URu}_{2} \mathrm{Si}_{2}$, Phys. Rev. B 41 (1990) 1829.

[312] M. Yokoyama, H. Amitsuka, S. Itoh, I. Kawasaki, K. Tenya, H. Yoshizawa, Neutron scattering study on competition between hidden order and antiferromagnetism in $\mathrm{U}\left(\mathrm{Ru}_{1-x} \mathrm{Rh}_{x}\right)_{2} \mathrm{Si}_{2}(x \leq 0.05)$, J. Phys. Soc. Jpn. 73 (2004) 545.

[313] N. Kanchanavatee, M. Janoschek, R. E. Baumbach, J. J. Hamlin, D. A. Zocco, K. Huang, M. B. Maple, Twofold enhancement of the hidden-order/large-moment antiferromagnetic phase boundary in the $\mathrm{URu}_{2-x} \mathrm{Fe}_{x} \mathrm{Si}_{2}$ system, Phys. Rev. B 84 (2011) 245122.

[314] N. Kanchanavatee, B. D. White, V. W. Burnett, M. B. Maple, Enhancement of the hidden order/large moment antiferromagnetic transition temperature in the $\mathrm{URu}_{2-x} \mathrm{Os}_{x} \mathrm{Si}_{2}$ system, Phil. Mag. 94 (2014) 3681.

[315] E. D. Bauer, V. S. Zapf, P.-C. Ho, N. P. Butch, E. J. Freeman, C. Sirvent, M. B. Maple, Non-Fermi-liquid behavior within the ferromagnetic phase in $\mathrm{URu}_{2-x} \mathrm{Re}_{x} \mathrm{Si}_{2}$, Phys. Rev. Lett. 94 (2005) 046401.

[316] N. P. Butch, M. B. Maple, Evolution of critical scaling behavior near a ferromagnetic quantum phase transition, Phys. Rev. Lett. 103 (2009) 076404.

[317] P. Das, N. Kanchanavatee, J. S. Helton, K. Huang, R. E. Baumbach, E. D. Bauer, B. D. White, V. W. Burnett, M. B. Maple, J. W. Lynn, M. Janoschek, Chemical pressure tuning of $\mathrm{URu}_{2} \mathrm{Si}_{2}$ via isoelectronic substitution of Ru with Fe, arXiv:1412.5642v1 [cond-mat.str-el].

[318] R. A. Fisher, S. Kim, Y. Wu, N. E. Phillips, M. W. McElfresh, M. S. Torikachvili, M. B. Maple, Specific heat of $\mathrm{URu}_{2} \mathrm{Si}_{2}$ : Effect of pressure and magnetic field on the magnetic and superconducting transitions, Physica B 163 (1990) 419.

[319] H. Amitsuka, K. Matsuda, I. Kawasaki, K. Tenya, M. Yokoyama, C. Sekine, N. Tateiwa, T. C. Kobayashi, S. Kawarazaki, H. Yoshizawa, Pressuretemperature phase diagram of the heavy-electron superconductor $\mathrm{URu}_{2} \mathrm{Si}_{2}$, J. Mag. Mag. Mater. 310 (2007) 214.

[320] E. Hassinger, G. Knebel, K. Izawa, P. Lejay, B. Salce, J. Flouquet, Temperature-pressure phase diagram of $\mathrm{URu}_{2} \mathrm{Si}_{2}$ from resistivity measurements and ac calorimetry: Hidden order and Fermi-surface nesting, Phys. Rev. B 77 (2008) 115117.

[321] J. R. Jeffries, N. P. Butch, B. T. Yukich, M. B. Maple, The evolution of the ordered states of single-crystal $\mathrm{URu}_{2} \mathrm{Si}_{2}$ under pressure, J. Phys.: Condens. Matter 20 (2008) 095225

[322] K. Matsuda, Y. Kohori, T. Kohara, Existence of line nodes in the superconducting energy gap of antiferromagnetic superconductor $\mathrm{URu}_{2} \mathrm{Si}_{2}$ : ${ }^{101}$ Ru NQR study, J. Phys. Soc. Jpn. 65 (1996) 679.

[323] Y. Kohori, K. Matsuda, T. Kohara, ${ }^{29} \mathrm{Si}$ NMR study of antiferromagnetic superconductor URu $\mathrm{Si}_{2}$, J. Phys. Soc. Jpn. 65 (1996) 1083.

[324] F. Morales, R. Escudero, Pseudogap and superconducting energy gap in single crystals of $\mathrm{URu}_{2} \mathrm{Si}_{2}$ by point contact spectroscopy, J. Low Temp. Phys. 154 (2009) 68

[325] C. Bergemann, S. R. Julian, G. J. McMullan, B. K. Howard, G. G. Lonzarich, P. Lejay, J. P. Brison, J. Flouquet, Quantum oscillations in $\mathrm{URu}_{2} \mathrm{Si}_{2}$, Physica B 230-232 (1997) 348 .

[326] H. Ohkuni, Y. Inada, Y. Tokiwa, K. Sakurai, R. Settai, T. Honma, Y. Haga, E. Yamamoto, Y. Ōnuki, H. Yamagami, S. Takahashi, T. Yanagisawa, Fermi surface properties and de Haas-van Alphen oscillation in both the normal and superconducting mixed states of $\mathrm{URu}_{2} \mathrm{Si}_{2}$, Phil. Mag. B 79 (1999) 1045.

[327] H. Shishido, K. Hashimoto, T. Shibauchi, T. Sasaki, H. Oizumi, N. Kobayashi, T. Takamasu, K. Takehana, Y. Imanaka, T. D. Matsuda, Y. Haga, Y. Onuki, Y. Matsuda, Possible phase transition deep inside the hidden order phase of ultraclean $\mathrm{URu}_{2} \mathrm{Si}_{2}$, Phys. Rev. Lett. 102 (2009) 156403.

[328] Y. Kasahara, T. Iwasawa, H. Shishido, T. Shibauchi, K. Behnia, Y. Haga, T. D. Matsuda, Y. Onuki, M. Sigrist, Y. Matsuda, Exotic superconducting properties in the electron-hole-compensated heavy-fermion "semimetal" URu $\mathrm{Si}_{2}$, Phys. Rev. Lett. 99 (2007) 116402.

[329] K. Yano, T. Sakakibara, T. Tayama, M. Yokoyama, H. Amitsuka, Y. Homma, P. Miranović, M. Ichioka, Y. Tsutsumi, K. Machida, Fieldangle-dependent specific heat measurements and gap determination of a heavy fermion superconductor $\mathrm{URu}_{2} \mathrm{Si}_{2}$, Phys. Rev. Lett. 100 (2008) 017004

[330] Y. Kasahara, H. Shishido, T. Shibauchi, Y. Haga, T. D. Matsuda, Y. Onuki, Y. Matsuda, Superconducting gap structure of heavy-fermion compound $\mathrm{URu}_{2} \mathrm{Si}_{2}$ determined by angle-resolved thermal conductivity, New J. Phys. 11 (2009) 055061.

[331] Y. Matsuda, K. Izawa, I. Vekhter, Nodal structure of unconventional superconductors probed by angle resolved thermal transport measurements, J. Phys.: Condens. Matter 18 (2006) R705.

[332] E. R. Schemm, R. E. Baumbach, P. H. Tobash, F. Ronning, E. D. Bauer, A. Kapitulnik, Evidence for broken time-reversal symmetry in the superconducting phase of $\mathrm{URu}_{2} \mathrm{Si}_{2}$, arXiv: $1410.1479 \mathrm{v} 1$ [cond-mat.supr-con].

[333] P. Visani, Y. Dalichaouch, M. A. Lopez de la Torre, B. W. Lee, C. L. Seaman, M. B. Maple, Logarithmic ac response in the heavy-fermion superconductor $\mathrm{URu}_{2} \mathrm{Si}_{2}$, Phys. Rev. B: Rapid Comm. 49 (1994) 4376.

[334] R. Okazaki, Y. Kasahara, H. Shishido, M. Konczykowski, K. Behnia, Y. Haga, T. D. Matsuda, Y. Onuki, T. Shibauchi, Y. Matsuda, Flux line lattice melting and the formation of a coherent quasiparticle Bloch state in the ultraclean $\mathrm{URu}_{2} \mathrm{Si}_{2}$ superconductor, Phys. Rev. Lett. 100 (2008) 037004.

[335] G. Oomi, T. Kagayama, K. Nishimura, S. W. Yun, Y. Ōnuki, Electrical resistivity of single crystalline $\mathrm{UGe}_{2}$ at high pressure and high magnetic field, Physica B 206-207 (1995) 515.

[336] A. Huxley, E. Ressouche, B. Grenier, D. Aoki, J. Flouquet, C. Pfleiderer, The co-existence of superconductivity and ferromagnetism in actinide compounds, J. Phys.: Condens. Matter 15 (2003) S1945.

[337] C. Pfleiderer, A. D. Huxley, Pressure dependence of the magnetization in the ferromagnetic superconductor $\mathrm{UGe}_{2}$, Phys. Rev. Lett. 89 (2002) 147005

[338] E. D. Bauer, R. P. Dickey, V. S. Zapf, M. B. Maple, Coexistence of superconductivity and ferromagnetism in polycrystalline $\mathrm{UGe}_{2}$, J. Phys.: Condens. Matter 13 (2001) L759.

[339] R. Vollmer, C. Pfleiderer, H. v. Löhneysen, E. D. Bauer, M. B. Maple, Low temperature specific heat of polycrystalline $\mathrm{UGe}_{2}$ at high pressure, Physica B 312-313 (2002) 112.

[340] A. de Visser, N. T. Huy, A. Gasparini, D. E. de Nijs, D. Andreica, C. Baines, A. Amato, Muon spin rotation and relaxation in the superconducting ferromagnet UCoGe, Phys. Rev. Lett. 102 (2009) 167003.

[341] C. Stock, D. A. Sokolov, P. Bourges, P. H. Tobash, K. Gofryk, F. Ronning, E. D. Bauer, K. C. Rule, A. D. Huxley, Anisotropic critical magnetic fluctuations in the ferromagnetic superconductor UCoGe, Phys. Rev. Lett. 107 (2011) 187202.

[342] Y. Ihara, T. Hattori, K. Ishida, Y. Nakai, E. Osaki, K. Deguchi, N. K. Sato, I. Satoh, Anisotropic magnetic fluctuations in the ferromagnetic superconductor UCoGe studied by direction-dependent ${ }^{59} \mathrm{Co}$ NMR measurements, Phys. Rev. Lett. 105 (2010) 206403.

[343] E. Hassinger, D. Aoki, G. Knebel, J. Floquet, Pressure-temperature phase diagram of polycrystalline UCoGe studied by resistivity measurement, J. Phys. Soc. Jpn. 77 (2008) 073703.

[344] K. Huang, J. J. Hamlin, R. E. Baumbach, M. Janoschek, N. Kanchanavatee, D. A. Zocco, F. Ronning, M. B. Maple, Ferromagnetic quantum critical point in $\mathrm{UCo}_{1-x} \mathrm{Fe}_{x} \mathrm{Ge}$, Phys. Rev. B 87 (2013) 054513.

[345] H. H. Hill, B. T. Matthias, Study of the superconductivity of the intermetallic compounds $\mathrm{U}_{6} \mathrm{Mn}, \mathrm{U}_{6} \mathrm{Fe}, \mathrm{U}_{6} \mathrm{Co}$, and $\mathrm{U}_{6} \mathrm{Ni}$ and alloys formed between them, Phys. Rev. 168 (1968) 464.

[346] O. T. Valls, Z. Tešanović, Superconductivity in almost-localized Fermi liquids: Application to heavy-fermion compounds, Phys. Rev. Lett. 53 
(1984) 1497

[347] M. B. Maple, J. W. Chen, S. E. Lambert, Z. Fisk, J. L. Smith, H. R. Ott, J. S. Brooks, M. J. Naughton, Upper critical magnetic field of the heavy-fermion superconductor $\mathrm{UBe}_{13}$, Phys. Rev. Lett. 54 (1985) 477.

[348] N. Wakeham, N. Ni, E. D. Bauer, J. D. Thompson, E. Tegtmeier, F. Ronning, Magnetism and superconductivity in $\mathrm{U}_{2} \mathrm{Pt}_{x} \mathrm{Rh}_{1-x} \mathrm{C}_{2}$, Phys. Rev. B 91 (2015) 024408.

[349] J. D. Thompson, G. P. Meisner, Pressure dependence of the electrical resistivity and $T_{c}$ of the "nearly-heavy-fermion" superconductor $\mathrm{U}_{2} \mathrm{PtC}_{2}$, Physica 130B (1985) 168.

[350] D. Einzel, P. J. Hirschfeld, F. Gross, B. S. Chandrasekhar, K. Andres, H. R. Ott, J. Beuers, Z. Fisk, J. L. Smith, Magnetic field penetration depth in the heavy-electron superconductor $\mathrm{UBe}_{13}$, Phys. Rev. Lett. 56 (1986) 2513

[351] D. E. MacLaughlin, C. Tien, W. G. Clark, M. D. Lan, Z. Fisk, J. L. Smith, H. R. Ott, Nuclear magnetic resonance and heavy-fermion superconductivity in (U,Th)Be ${ }_{13}$, Phys. Rev. Lett. 53 (1984) 1833.

[352] Y. Dalichaouch, B. W. Lee, S. E. Lambert, M. B. Maple, J. L. Smith, Z. Fisk, Upper critical magnetic field of the heavy-electron superconductors $\mathrm{U}_{1-x} \mathrm{Th}_{x} \mathrm{Be}_{13}(x=0$ and $2.9 \%$ ) doped with paramagnetic $\mathrm{Gd}$ and other rare-earth ions, Phys. Rev. B 43 (1991) 299.

[353] P. C. Canfield, Fishing the Fermi sea, Nature Phys. 4 (2008) 167.

[354] M. B. Maple, P.-C. Ho, V. S. Zapf, N. A. Frederick, E. D. Bauer, W. M. Yuhasz, F. M. Woodward, J. W. Lynn, Heavy fermion superconductivity in the filled skutterudite compound $\mathrm{PrOs}_{4} \mathrm{Sb}_{12}$, J. Phys. Soc. Jpn. 71 (2002) 23.

[355] M.-A. Measson, D. Braithwaite, J. Flouquet, G. Seyfarth, J. P. Brison, E. Lhotel, C. Paulsen, H. Sugawara, H. Sato, Superconducting phase diagram of the filled skuterrudite $\mathrm{PrOs}_{4} \mathrm{Sb}_{12}$, Phys. Rev. B 70 (2004) 064516.

[356] M. B. Maple, Z. Henkie, W. M. Yuhasz, P.-C. Ho, T. Yanagisawa, T. A. Sayles, N. P. Butch, J. R. Jeffries, A. Pietraszko, Strongly correlated electron phenomena in Pr-based filled skutterudite compounds, J. Mag. Mag. Mater. 310 (2007) 182

[357] M. B. Maple, N. A. Frederick, P.-C. Ho, W. M. Yuhasz, T. Yanagisawa, Unconventional superconductivity and heavy fermion behavior in $\mathrm{PrOs}_{4} \mathrm{Sb}_{12}$, J. Supercond. Novel Mag. 19 (2006) 299.

[358] E. A. Goremychkin, R. Osborn, E. D. Bauer, M. B. Maple, N. A. Frederick, W. M. Yuhasz, F. M. Woodward, J. W. Lynn, Crystal field potential of $\mathrm{PrOs}_{4} \mathrm{Sb}_{12}$ : Consequences for superconductivity, Phys. Rev. Lett. 93 (2004) 157003.

[359] Y. Aoki, T. Namiki, S. Ohsaki, S. R. Saha, H. Sugawara, H. Sato, Thermodynamical study on the heavy-fermion superconductor $\mathrm{PrOs}_{4} \mathrm{Sb}_{12}$ : Evidence for field-induced phase transition, J. Phys. Soc. Jpn. 71 (2002) 2098

[360] P.-C. Ho, V. S. Zapf, E. D. Bauer, N. A. Frederick, M. B. Maple, G. Giester, P. Rogl, S. T. Berger, C. H. Paul, E. Bauer, Superconducting and normal state properties of the heavy fermion compound $\mathrm{PrOs}_{4} \mathrm{Sb}_{12}$, Int J. Mod. Phys. B 16 (2002) 3008.

[361] P.-C. Ho, N. A. Frederick, V. S. Zapf, E. D. Bauer, T. D. Do, M. B. Maple, A. D. Christianson, A. H. Lacerda, High-field ordered and superconducting phases in the heavy-fermion compound $\mathrm{PrOs}_{4} \mathrm{Sb}_{12}$, Phys. Rev. B 67 (2003) 180508(R).

[362] T. Tayama, T. Sakakibara, H. Sugawara, Y. Aoki, H. Sato, Magnetic phase diagram of the heavy fermion superconductor $\mathrm{PrOs}_{4} \mathrm{Sb}_{12}$, J. Phys. Soc. Jpn. 72 (2003) 1516.

[363] M. Kohgi, K. Iwasa, M. Nakajima, N. Metoki, S. Araki, N. Bernhoeft, J.-M. Mignot, A. Gukasov, H. Sato, Y. Aoki, H. Sugawara, Evidence for magnetic-field-induced quadrupolar ordering in the heavy-fermion superconductor $\mathrm{PrOs}_{4} \mathrm{Sb}_{12}$, J. Phys. Soc. Jpn. 72 (2003) 1002

[364] D. E. MacLaughlin, J. E. Sonier, R. H. Heffner, O. O. Bernal, B.L. Young, M. S. Rose, G. D. Morris, E. D. Bauer, T. D. Do, M. B. Maple, Muon spin relaxation and isotropic pairing in superconducting $\mathrm{PrOs}_{4} \mathrm{Sb}_{12}$, Phys. Rev. Lett. 89 (2002) 157001.

[365] H. Suderow, S. Vieira, J. D. Strand, S. Bud'ko, P. C. Canfield, Very-lowtemperature tunneling spectroscopy in the heavy-fermion superconductor $\mathrm{PrOs}_{4} \mathrm{Sb}_{12}$, Phys. Rev. B 69 (2004) 060504(R).

[366] A. D. Huxley, M.-A. Measson, K. Izawa, C. D. Dewhurst, R. Cubitt, B. Grenier, H. Sugawara, J. Flouquet, Y. Matsuda, H. Sato, Flux-line lattice distortion in $\mathrm{PrOs}_{4} \mathrm{Sb}_{12}$, Phys. Rev. Lett. 93 (2004) 187005

[367] L. Shu, D. E. MacLaughlin, W. P. Beyermann, R. H. Heffner, G. D.
Morris, O. O. Bernal, F. D. Callaghan, J. E. Sonier, W. M. Yuhasz, N. A. Frederick, M. B. Maple, Penetration depth, multiband superconductivity, and absence of muon-induced perturbation in superconducting $\mathrm{PrOs}_{4} \mathrm{Sb}_{12}$, Phys. Rev. B 79 (2009) 174511

[368] G. Seyfarth, J. P. Brison, M.-A. Méasson, J. Flouquet, K. Izawa, Y. Matsuda, H. Sugawara, H. Sato, Multiband superconductivity in the heavy fermion compound $\mathrm{PrOs}_{4} \mathrm{Sb}_{12}$, Phys. Rev. Lett. 95 (2005) 107004

[369] G. Seyfarth, J. P. Brison, M.-A. Méasson, D. Braithwaite, G. Lapertot, J. Flouquet, Superconducting $\mathrm{PrOs}_{4} \mathrm{Sb}_{12}$ : A thermal conductivity study, Phys. Rev. Lett. 97 (2006) 236403.

[370] R. W. Hill, S. Li, M. B. Maple, L. Taillefer, Multiband order parameters for the $\mathrm{PrOs}_{4} \mathrm{Sb}_{12}$ and $\mathrm{PrRu}_{4} \mathrm{Sb}_{12}$ skutterudite superconductors from thermal conductivity measurements, Phys. Rev. Lett. 101 (2008) 237005

[371] Y. Aoki, A. Tsuchiya, T. Kanayama, S. R. Saha, H. Sugawara, H. Sato, W. Higemoto, A. Koda, K. Ohishi, K. Nishiyama, R. Kadono, Time-reversal symmetry-breaking superconductivity in heavy-fermion $\mathrm{PrOs}_{4} \mathrm{Sb}_{12}$ detected by muon-spin relaxation, Phys. Rev. Lett. 91 (2003) 067003.

[372] E. Bauer, A. Grytsiv, X.-Q. Chen, N. Melnychenko-Koblyuk, G. Hilscher, H. Kaldarar, H. Michor, E. Royanian, G. Giester, M. Rotter, R. Podloucky, P. Rogl, Superconductivity in novel Ge-based skutterudites: (Sr,Ba)Pt ${ }_{4} \mathrm{Ge}_{12}$, Phys. Rev. Lett. 99 (2007) 217001

[373] R. Gumeniuk, W. Schnelle, H. Rosner, M. Nicklas, A. Leithe-Jasper, Y. Grin, Superconductivity in the platinum germanides $M \mathrm{Pt}_{4} \mathrm{Ge}_{12}(M$ $=$ rare-earth or alkaline-earth metal) with filled skutterudite structure, Phys. Rev. Lett. 100 (2008) 017002.

[374] A. Maisuradze, M. Nicklas, R. Gumeniuk, C. Baines, W. Schnelle, H. Rosner, A. Leithe-Jasper, Y. Grin, R. Khasanov, Superfluid density and energy gap function of superconducting $\mathrm{PrPt}_{4} \mathrm{Ge}_{12}$, Phys. Rev. Lett. 103 (2009) 147002.

[375] A. Maisuradze, W. Schnelle, R. Khasanov, R. Gumeniuk, M. Nicklas, H. Rosner, A. Leithe-Jasper, Y. Grin, A. Amato, P. Thalmeier, Evidence for time-reversal symmetry breaking in superconducting $\mathrm{PrPt}_{4} \mathrm{Ge}_{12}$, Phys. Rev. B 82 (2010) 024524.

[376] L. S. Sharath Chandra, M. K. Chattopadhyay, S. B. Roy, Evidence for two superconducting gaps in the unconventional superconductor $\mathrm{PrPt}_{4} \mathrm{Ge}_{12}$, Phil. Mag. 92 (2012) 3866.

[377] Y. Nakamura, H. Okazaki, R. Yoshida, T. Wakita, H. Takeya, K. Hirata, M. Hirai, Y. Muraoka, T. Yokoya, Comparative photoemission studies on the superconducting gap of the filled skutterudite superconductors $\mathrm{LaPt}_{4} \mathrm{Ge}_{12}$ and $\mathrm{PrPt}_{4} \mathrm{Ge}_{12}$, Phys. Rev. B 86 (2012) 014521.

[378] J. L. Zhang, Y. Chen, L. Jiao, R. Gumeniuk, M. Nicklas, Y. H. Chen, L. Yang, B. H. Fu, W. Schnelle, H. Rosner, A. Leithe-Jasper, Y. Grin, F. Steglich, H. Q. Yuan, Multiband superconductivity in $\mathrm{PrPt}_{4} \mathrm{Ge}_{12}$ single crystals, Phys. Rev. B 87 (2013) 064502.

[379] K. Huang, L. Shu, I. K. Lum, B. D. White, M. Janoschek, D. Yazici, J. J. Hamlin, D. A. Zocco, P.-C. Ho, R. E. Baumbach, M. B. Maple, Probing the superconductivity of $\mathrm{PrPt}_{4} \mathrm{Ge}_{12}$ through Ce substitution, Phys. Rev. B 89 (2014) 035145

[380] M. Toda, H. Sugawara, K. ichi Magishi, T. Saito, K. Koyama, Y. Aoki, H. Sato, Electrical, magnetic and NMR studies of Ge-based filled skutterudites $R \mathrm{Pt}_{4} \mathrm{Ge}_{12}(R=\mathrm{La}, \mathrm{Ce}, \mathrm{Pr}, \mathrm{Nd})$, J. Phys. Soc. Jpn. 77 (2008) 124702

[381] T. Onimaru, K. T. Matsumoto, Y. F. Inoue, K. Umeo, Y. Saiga, Y. Matsushita, R. Tamura, K. Nishimoto, I. Ishii, T. Suzuki, T. Takabatake, Superconductivity and structural phase transitions in caged compounds $\mathrm{RT}_{2} \mathrm{Zn}_{20}(\mathrm{R}=\mathrm{La}, \mathrm{Pr}, \mathrm{T}=\mathrm{Ru}, \mathrm{Ir})$, J. Phys. Soc. Jpn. 79 (2010) 033704.

[382] T. Onimaru, K. T. Matsumoto, Y. F. Inoue, K. Umeo, T. Sakakibara, Y. Karaki, M. Kubota, T. Takabatake, Antiferroquadrupolar ordering in a Pr-based superconductor PrIr ${ }_{2} Z_{20}$, Phys. Rev. Lett. 106 (2011) 177001.

[383] K. Iwasa, H. Kobayashi, T. Onimaru, K. T. Matsumoto, N. Nagasawa, T. Takabatake, S. Ohira-Kawamura, T. Kikuchi, Y. Inamura, K. Nakajima, Well-defined crystal field splitting schemes and non-Kramers doublet ground states of $f$ electrons in $\operatorname{Pr} T_{2} \mathrm{Zn}_{20}(T=\mathrm{Ir}, \mathrm{Rh}$, and $\mathrm{Ru}), \mathrm{J}$. Phys. Soc. Jpn. 82 (2013) 043707.

[384] M. Matsushita, J. Sakaguchi, Y. Taga, M. Ohya, S. Yoshiuchi, H. Ota, Y. Hirose, K. Enoki, F. Honda, K. Sugiyama, M. Hagiwara, K. Kindo, T. Tanaka, Y. Kubo, T. Takeuchi, R. Settai, Y. Ōnuki, Fermi surface property and characteristic crystalline electric field effect in $\operatorname{PrIr}_{2} \mathrm{Zn}_{20}$, J. Phys. Soc. Jpn. 80 (2011) 074605. 
[385] T. Onimaru, N. Nagasawa, K. T. Matsumoto, K. Wakiya, K. Umeo, S. Kittaka, T. Sakakibara, Y. Matsushita, T. Takabatake, Simultaneous superconducting and antiferroquadrupolar transitions in $\operatorname{PrRh}_{2} \mathrm{Zn}_{20}$, Phys. Rev. B 86 (2012) 184426.

[386] M. Koseki, Y. Nakanishi, K. Deto, G. Koseki, R. Kashiwazaki, F. Shichinomiya, M. Nakamura, M. Yoshizawa, A. Sakai, S. Nakatsuji, Ultrasonic investigation on a cage structure compound $\operatorname{PrTi}_{2} \mathrm{Al}_{20}$, J. Phys. Soc. Jpn. 80 (2011) SA049.

[387] M. Matsunami, M. Taguchi, A. Chainani, R. Eguchi, M. Oura, A. Sakai, S. Nakatsuji, S. Shin, Kondo resonance in $\mathrm{PrTi}_{2} \mathrm{Al}_{20}$ : Photoemission spectroscopy and single-impurity Anderson model calculations, Phys. Rev. B 84 (2011) 193101.

[388] V. W. Burnett, D. Yazici, B. D. White, N. R. Dilley, A. J. Friedman, B. Brandom, M. B. Maple, Structure and physical properties of $R T_{2} \mathrm{Cd}_{20}$ ( $R=$ rare earth, $T=\mathrm{Ni}, \mathrm{Pd}$ ) compounds with the $\mathrm{CeCr}_{2} \mathrm{Al}_{20}$-type structure, J. Solid State Chem. 215 (2014) 114.

[389] D. Yazici, B. D. White, P.-C. Ho, N. Kanchanavatee, K. Huang, A. J. Friedman, A. S. Wong, V. W. Burnett, N. R. Dilley, M. B. Maple, Investigation of magnetic order in $\mathrm{Sm}_{2} \mathrm{Z}_{2} \mathrm{Zn}_{20}(T \mathrm{r}=\mathrm{Fe}, \mathrm{Co}$, and $\mathrm{Ru})$ and $\mathrm{Sm} \mathrm{r}_{2} \mathrm{Cd}_{20}(\mathrm{Tr}=\mathrm{Ni}$ and Pd), Phys. Rev. B 90 (2014) 144406.

[390] D. Yazici, T. Yanagisawa, B. D. White, M. B. Maple, Nonmagnetic ground state in the cubic compounds $\operatorname{PrNi}_{2} \mathrm{Cd}_{20}$ and $\operatorname{PrPd}_{2} \mathrm{Cd}_{20}$, submitted to Phys. Rev. B XX (2015) XXXXXX

[391] Z. Fisk, M. B. Maple, On the existence of heavy fermion ytterbium compounds, J. Alloys and Comp. 183 (1992) 303.

[392] A. H. Nevidomskyy, P. Coleman, Layered Kondo lattice model for quantum critical $\beta$-YbAlB 4 , Phys. Rev. Lett. 102 (2009) 077202.

[393] E. C. T. O'Farrell, D. A. Tompsett, S. E. Sebastian, N. Harrison, C. Capan, L. Balicas, K. Kuga, A. Matsuo, K. Kindo, M. Tokunaga, S. Nakatsuji, G. Csányi, Z. Fisk, M. L. Sutherland, Role of $f$ electrons in the Fermi surface of the heavy fermion superconductor $\beta$-YbAlB 4 , Phys. Rev. Lett. 102 (2009) 216402.

[394] E. C. T. O'Farrell, Y. Matsumoto, S. Nakatsuji, Evolution of $c$ - $f$ hybridization and two-component Hall effect in $\beta$-YbAlB 4 , Phys. Rev. Lett. 109 (2012) 176405.

[395] Y. Matsumoto, S. Nakatsuji, K. Kuga, Y. Karaki, N. Horie, Y. Shimura, T. Sakakibara, A. H. Nevidomskyy, P. Coleman, Quantum criticality without tuning in the mixed valence compound $\beta$-YbAlB 4 , Science 331 (2011) 316.

[396] A. Ramires, P. Coleman, A. H. Nevidomskyy, A. M. Tsvelik, $\beta$-YbAlB 4 : A critical nodal metal, Phys. Rev. Lett. 109 (2012) 176404.

[397] M. Okawa, M. Matsunami, K. Ishizaka, R. Eguchi, M. Taguchi, A. Chainani, Y. Takata, M. Yabashi, K. Tamasaku, Y. Nishino, T. Ishikawa, K. Kuga, N. Horie, S. Nakatsuji, S. Shin, Strong valence fluctuation in the quantum critical heavy fermion superconductor $\beta$ $\mathrm{YbAlB}_{4}$ : A hard x-ray photoemission study, Phys. Rev. Lett. 104 (2010) 247201.

[398] S. Watanabe, K. Miyake, Quantum valence criticality as an origin of unconventional critical phenomena, Phys. Rev. Lett. 105 (2010) 186403.

[399] L. M. Holanda, J. M. Vargas, W. Iwamoto, C. Rettori, S. Nakatsuji, K. Kuga, Z. Fisk, S. B. Oseroff, P. G. Pagliuso, Quantum critical Kondo quasiparticles probed by ESR in $\beta$-YbAlB 4 , Phys. Rev. Lett. 107 (2011) 026402.

[400] A. Ramires, P. Coleman, Theory of the electron spin resonance in the heavy fermion metal $\beta$-YbAlB 4 , Phys. Rev. Lett. 112 (2014) 116405.

[401] Y. Machida, K. Tomokuni, C. Ogura, K. Izawa, K. Kuga, S. Nakatsuji, G. Lapertot, G. Knebel, J.-P. Brison, J. Flouquet, Thermoelectric response near a quantum critical point of $\beta$ - $\mathrm{YbAlB}_{4}$ and $\mathrm{YbRh}_{2} \mathrm{Si}_{2}$ : A comparative study, Phys. Rev. Lett. 109 (2012) 156405.

[402] A. J. Arko, M. B. Brodsky, W. J. Nellis, Spin fluctuations in plutonium and other actinide metals and compounds, Phys. Rev. B 5 (1972) 4564.

[403] R. J. Trainor, M. B. Brodsky, G. S. Knapp, Calorimetric study of the intermetallic compounds $\mathrm{UAl}_{2}$ and $\mathrm{PuAl}_{2}$, in: H. Blank, R. Lindner (Eds.), 5th International Conference on Plutonium and Other Actinides, NorthHolland, 1976, p. 475

[404] J. D. Thompson, M. Nicklas, V. A. Sidorov, E. D. Bauer, R. Movshovich, N. J. Curro, J. L. Sarrao, Interplay of magnetism, structure and superconductivity in heavy-fermion systems CeMIn 5 and $\mathrm{PuMGa}_{5}$, J. Alloys Comp. 408-412 (2006) 16.

[405] D. C. Johnston, The puzzle of high temperature superconductivity in layered iron pnictides and chalcogenides, Adv. Phys. 59 (2010) 803
[406] P. J. Hirschfeld, M. M. Korshunov, I. I. Mazin, Gap symmetry and structure of Fe-based superconductors, Rep. Prog. Phys. 74 (2011) 124508.

[407] G. R. Stewart, Superconductivity in iron compounds, Rev. Mod. Phys. 83 (2011) 1589.

[408] A. Chubukov, Pairing mechanism in Fe-based superconductors, Ann. Rev. 3 (2012) 57

[409] E. Dagotto, Correlated electrons in high-temperature superconductors, Rev. Mod. Phys. 66 (1994) 763.

[410] C. C. Tsuei, J. R. Kirtley, Pairing symmetry in cuprate superconductors, Rev. Mod. Phys. 72 (2000) 969.

[411] P. A. Lee, N. Nagaosa, X.-G. Wen, Doping a Mott insulator: Physics of high-temperature superconductivity, Rev. Mod. Phys. 78 (2006) 17.

[412] D. N. Basov, A. V. Chubukov, Manifesto for a higher $T_{c}$, Nat. Phys. 7 (2011) 272.

[413] M. R. Norman, Unconventional superconductivity, in: K. H. Bennemann, J. B. Ketterson (Eds.), Novel Superfluids, Vol. 2, Oxford University Press, 2014.

[414] Y. Kamihara, T. Watanabe, M. Hirano, H. Hosono, Iron-based layered superconductor $\mathrm{La}\left[\mathrm{O}_{1-x} \mathrm{~F}_{x}\right] \mathrm{FeAs}(x=0.05-0.12)$ with $T_{c}=26 \mathrm{~K}$, J. Am. Chem. Soc. 130 (2008) 3296.

[415] J. Rossat-Mignod, L. P. Regnault, C. Vettier, P. Burlet, J. Y. Henry, G. Lapertot, Investigation of the spin dynamics in $\mathrm{YBa}_{2} \mathrm{Cu}_{3} \mathrm{O}_{6+x}$ by inelastic neutron scattering, Physica B 169 (1991) 58.

[416] H. A. Mook, M. Yethiraj, G. Aeppli, T. E. Mason, T. Armstrong, Polarized neutron determination of the magnetic excitations in $\mathrm{YBa}_{2} \mathrm{Cu}_{3} \mathrm{O}_{7}$, Phys. Rev. Lett. 70 (1993) 3490.

[417] H. F. Fong, B. Keimer, P. W. Anderson, D. Reznik, F. Doğan, I. A. Aksay, Phonon and magnetic neutron scattering at $41 \mathrm{meV}$ in $\mathrm{YBa}_{2} \mathrm{Cu}_{3} \mathrm{O}_{7}$, Phys. Rev. Lett. 75 (1995) 316

[418] H. F. Fong, P. Bourges, Y. Sidis, L. P. Regnault, A. Ivanov, G. D. Guk, N. Koshizuka, B. Keimer, Neutron scattering from magnetic excitations in $\mathrm{Bi}_{2} \mathrm{Sr}_{2} \mathrm{CaCu}_{2} \mathrm{O}_{8+\delta}$, Nature 398 (1999) 588 .

[419] D. S. Inosov, J. T. Park, P. Bourges, D. L. Sun, Y. Sidis, A. Schneidewind, K. Hradil, D. Haug, C. T. Lin, B. Keimer, V. Hinkov, Normalstate spin dynamics and temperature-dependent spin-resonance energy in optimally doped $\mathrm{BaFe}_{1.85} \mathrm{Co}_{0.15} \mathrm{As}_{2}$, Nat. Phys. 6 (2010) 178.

[420] T. Moriya, K. Ueda, Antiferromagnetic spin fluctuation and superconductivity, Rep. Prog. Phys. 66 (2003) 1299.

[421] N. J. Curro, T. Caldwell, E. D. Bauer, L. A. Morales, M. J. Graf, Y. Bang, A. V. Balatsky, J. D. Thompson, J. L. Sarrao, Unconventional superconductivity in PuCoGa5, Nature 434 (2005) 622. 